\title{
The structure and phase transitions in \\ polymer blends, diblock copolymers and liquid crystalline polymers: the Landau-Ginzburg approach
}

\author{
by \\ Robert Hołyst and T.A.Vilgisł \\ Institute of Physical Chemistry PAS and College of Science, Dept. III, \\ Kasprzaka 44/52, 01224 Warsaw, Poland \\ $\ddagger$ Max-Planck-Institut für Polymerforschung, P.O. Box 3148, D-55021 Mainz, F.R.G.
}

\begin{abstract}
The polymer systems are discussed in the framework of the Landau-Ginzburg model. The model is derived from the mesoscopic Edwards hamiltonian via the conditional partition function. We discuss flexible, semiflexible and rigid polymers. The following systems are studied: polymer blends, flexible diblock and multi-block copolymer melts, random copolymer melts, ring polymers, rigid-flexible diblock copolymer melts, mixtures of copolymers and homopolymers and mixtures of liquid crystalline polymers. Three methods are used to study the systems: mean-field model, self consistent one-loop approximation and self consistent field theory. The following problems are studied and discussed: the phase diagrams, scattering intensities and correlation functions, single chain statistics and behavior of single chain close to critical points, fluctuations induced shift of phase boundaries. In particular
\end{abstract}


we shall discuss shrinking of the polymer chains close to the critical point in polymer blends, size of the Ginzburg region in polymer blends and shift of the critical temperature. In the rigid flexible diblock copolymers we shall discuss the density nematic order parameter correlation function. The correlation functions in this system are found to oscillate with the characteristic period equal to the length of the rigid part of the diblock copolymer. The density and nematic order parameter measured along the given direction are anticorrelated. In the flexible diblock copolymer system we shall discuss various phases including the double diamond and gyroid structures. The single chain statistics in the disordered phase of flexible diblock copolymer system is shown to deviate from the Gaussian statistics due to fluctuations. In the one loop approximation one shows that the diblock copolymer chain is stretched in the point where two incompatible blocks meet but also that each block shrinks close to the microphase separation transition. The stretching outweights shrinking and the net result is the increase of the radius of gyration about the Gaussian value. Certain properties of the homopolymer, copolymer system are discussed. The diblock copolymers solubilizes two incompatible homopolymers by forming a monolayer interfaces between them. The interface has the positive saddle splay modulus which means that the interfaces in the disordered phase should be characterized by the negative Gaussian curvature. We also show that in such a mixture the Lifshitz tricritical point is encountered. The properties of this unusual point are presented. The Lifshitz, equimaxima and disorder line are shown to provide a useful tool for studying local ordering in polymer mixtures. In the liquid crystalline mixtures the isotropic nematic phase transition is discussed. We concentrate on static, equilibrium properties of the polymer systems. 


\section{Introduction}

The introduction of the order parameter in physics was a milestone in the description of phase transitions. The same Landau-Ginzburg approach has been applied to such diverse phenomena as transitions in liquid crystals and superconductivity, liquid-solid or solid-solid phase transitions and transitions in the superfluid helium, ferromagnetic paramagnetic phase transition and phase transitions in polymer systems. The purpose of this review is the application of the Landau-Ginzburg model to polymer systems. Despite the obvious limitations of this approach (valid for weakly first order and continuous phase transitions) it provides plenty of information about the polymer systems such as: polymer blends, diblock copolymers and liquid crystalline polymers. In polymer systems the parameters characterizing the Landau-Ginzburg free energy can be directly related to the mesoscopic parameters of the polymer systems. The mesoscopic parameters characterizing for example the interactions are, on the other hand, related to the microscopic interaction parameters via the integrals of the direct corelation functions. Therefore the results obtained in the Landau-Ginzburg model shed light on the relation between the mesoscopic and/or microscopic parameters of the system and its behaviour.

The simplest polymer mixture is the A homopolymer, B homopolymer blend. The very fruitful approach to the study of the system near its consolute (critical) point is based on the mean field Flory-Huggins free energy ${ }^{1)}$ and the de Gennes random phase approximation $(\mathrm{RPA})^{2)}$ for the scattering intensity. Both can be stated in terms of the Landau-Ginzburg model as we show in Section III. RPA and Flory-Huggins approach are two compatible mean-field models. They are named mean-field since in both models only the most probable configurations of the polymer chain are taken into account and, more importantly, the critical long-wavelength fluctuations are neglected. It was de Gennes who pointed out ${ }^{3)}$ that the mean-field 
theory is rather good for high molecular mass polymer mixtures, in contrast to the low molecular mass mixtures, for which the mean-field theory breaks down close to the critical point. In fact, in the limit of $N_{A}, N_{B} \rightarrow \infty$, the mean-field theory is exact ${ }^{3-5)}$. The mean-field approach is quantitatively correct if the correlation length $\xi \sim\left(T / T_{c}-1\right)^{-1}$ is comparable or smaller than the typical length scale in the system i.e. the radius of gyration $\sim \sqrt{N}$. Comparing two length scales, for large $N$, we find that the mean-field theory breaks down very close to the critical point, i.e. for

$$
\left|T-T_{c}\right| / T_{c} \sim 1 / N
$$

This is the Ginzburg criterion. The region around the critical point where the mean field theory breaks down is called the Ginzburg region. The shift of the critical temperature, $T_{c}$, with respect to $T_{c}^{m f}$ (calculated in the Flory Huggins model) is

$$
\left|T_{c}-T_{c}^{m f}\right| / T_{c}^{m f} \sim 1 / \sqrt{N}
$$

The shift, induced by fluctuations, is obtained in the one-loop approximation ${ }^{6,7)}$. It is larger than the Ginzburg region itself. The derivation of $\operatorname{Eq}(1.2)$ is based on the study of the scattering intensity, $I(q)$, where $q$ is the scattering wavevector. For $T \rightarrow T_{c}$ and $q \rightarrow 0$, we expect $I^{-1}(0) \sim\left(T / T_{c}-1\right)^{-\gamma}$, where $\gamma=1$ in the mean-field theory ${ }^{6,7)}$. The breakdown of this relation is usually used in experiment to estimate the Ginzburg region ${ }^{7,8-13)}$ and therefore, we also base the calculations of the Ginzburg region on the scattering intensity ${ }^{14,15)}$. Sufficiently close to $T_{c}$, fluctuations become important and $\gamma$ changes from 1 to its universal value of 1.26. This change can be extracted if one plots $\log I(0)$ versus $\log \left|T / T_{c}-1\right|$.

Different polymers can be combined into single material in many ways and polymer blends find widely spread application. Many of the potential application depends on the morphology of the system which in turn depends on the single 
chain behaviour. Here we shall discuss the behavior of the single chain in the polymer blend ${ }^{16-19)}$ as well as in the diblock copolymer system ${ }^{20,21)}$. The chains shrink considerably at the approach to the critical point in homopolymer blends. Intuitively we can expect that when the correlation length becomes larger than the radius of gyration the chains demix on this scale ${ }^{18)}$. On the other hand the chains stretch in diblock copolymer system ${ }^{20,21)}$.

An A-B diblock copolymer is a polymer consisting of a sequence of A-type monomers chemically joined to a sequence of B-type monomers. Even a small amount of incompatibility (difference in interactions) between monomers $\mathrm{A}$ and monomers B can induce phase transitions in a mixture of homopolymers. However A-homopolymer and B-homopolymer are chemically joined in a diblock, so that a system of diblocks cannot undergo a macroscopic phase separation. Instead a number of order-disorder phase transitions take place in the system between the isotropic phase and spatially ordered phases in which A-rich and B-rich domains, of the size of a diblock copolymer, are periodically arranged in lamellar, hexagonal, bcc, lamellar-catenoid, double diamond (Fig.1) or gyroid structures (Fig.2). A Landau-Ginzburg model of the phase transitions in diblock copolymer system was first formulated by Leibler ${ }^{22)}$ and later refined by Fredrickson et $\mathrm{al}^{20,23)}$. Matsen and Schick $^{24,25)}$ applied the self consistent field theory ${ }^{26,27)}$ to the phase transitions in this system in the weak segregation limit ${ }^{28)}$, where the domain boundaries between the A rich and $\mathrm{B}$ rich domains are wide. The opposite case is the strong segregation regime with very sharp and narrow domain boundaries; here we consider only the former case. The theoretical studies of the system were greatly stimulated by experiments. The cylindrical and spherical and lamellar phases have been known for long time $^{29)}$, but only recently the novel bicontinuous structures have been discovered in the diblock copolymer systems ${ }^{30-32)}$ (Fig.1). In the polystyrene-polyisoprene 
system (Fig.3) the bicontinuous double diamond structure consists of two channels each of diamond symmetry separated by the surface (Fig.1), which as has been argued by Thomas et $\mathrm{al}^{32}$ ) is of constant mean curvature $^{33)}$.

The richness of the phase diagram and behavior of the polymer system is enhanced if we mix the diblock copolymers and homopolymers ${ }^{34-37)}$. Ternary mixtures of A,B homopolymers and A-B diblock copolymer ${ }^{34-36)}$ are similar in some respects to mixtures of oil, water and amphiphile ${ }^{38)}$. The copolymer acts as a surfactant for the A,B homopolymer blend, decreasing the surface tension between A-rich and B-rich phases as it accumulates on the interface between these two phases and reduces the number of energetically unfavorable contacts between the A-homopolymers and B-homopolymers. In spite of this similarity, the comopolymer is not generally as efficient a solubilizer of homopolymers as a good amphiphile is of oil and water ${ }^{35)}$. The analysis of the disordered system is based on the study of disorder ${ }^{39)}$, Lifshitz and equimaxima lines. The disorder line of the system is the locus of points at which all correlation functions in general no longer decay monotonically, but contain an exponentially damped oscillatory component. This oscillation reflects the tendency of the copolymer to order the A (B) monomers of the system. The Lifshitz line is the locus of points at which the peak in the structure function just begins to move off of zero wavevector. It therefore indicates the point at which oscillatory components begin to dominate the particular correlation function, in contrast to the disorder line which indicates the point at which oscillatory components appear in all correlation functions. The Lifshitz line of the structure function of all A monomers is quite close to the disorder line, indicating that once the tendency to order the system appears, it quickly dominates the response of this function. In contrast, the Lifshitz line associated with the structure function of those monomers located only on the homopolymers is, in a large part of the phase 
diagram, far from the disorder line. These results taken together indicates that the copolymer is rather ineffective in organizing the homopolymers, and much more efficient in organizing itself, an organization expressed most strongly in the lyotropic phases which easily exist in the complete absence of homopolymer. Beyond the disorder line the interface between the A-rich homopolymer B-rich homopolymer phase is not wetted by the disordered phase ${ }^{36)}$ so it consists of of a thin copolymer layer. In the disordered phase we expect that A-rich and B-rich domains are also separated by the thin copolymer layer. Assuming that the structure of the interface is the same as the internal interfaces in the disordered phase Matsen and Schick have calculated ${ }^{36)}$ the profiles and from them obtained the saddle-splay modulus for the internal intefaces. The modulus is positive, thus internal interfaces in the disordered phase of A,B homopolymers and $\mathrm{AB}$ diblock copolymers are characterized by the negative Gaussian curvature.

Random copolymers ${ }^{40)}$ are produced by polimerization reaction in the medium that contains two (or more) distinct monomer species. The chemical composition of the copolymers is dictated by a set of bimolecular reaction rates. The system is characterized by quenched disorder ${ }^{41-43)}$. The chain architecture is determined statistically by the conditional probability that A monomer follows B monomer. Under steady state conditions of the reaction this probability is independent of the location of the monomer in the chain. The structures formed in the random copolymer system depend on the structure of the chain and thus on the polimerization process, thus the phase diagram can be a useful guide for the design of new polymeric materials.

In the multiblock copolymer system ${ }^{44)}$ new ingredients enter into physics in comparison to diblock copolymers. In the latter the junction of the blocks of a given copolymer is constrained to one A/B block interface; in the former bridging 
and looping is possible. In the loop configuration of the AB multi-block copolymer system both junctions can reside on the same interface (Fig.4). In the bridge configuration the middle B block spans over one B-rich lamella and joins two A-rich lamellas. In many material properties the bridge configurations are important ${ }^{45)}$. The bridging fraction thus provides important information for the design of new materials.

Ring homopolymers or ring diblock copolymers provide yet another example of the influence of chain architecture on the structure and phase diagram of polymer system. In the AB ring polymer the A block is connected with the B block at two junction points, therefore the chain has no free ends. The topological constraint introduced by this chain architecture, prevents the different chains from entangledment or self-knotting (Fig.5). Thus the statistics of the rings is not Gaussian and the size of a ring is intermediate between the Gaussian and collapsed size. For the ring homopolymer in the melt the scaling arguments ${ }^{46 a)}$ show that the radius of gyration scales with the polimerization index, $N$, as $N^{\nu}$, with $\nu=0.4$. The application of RPA to the Edwards hamiltonian with the specific constraint of no knots gives the more accurate value ${ }^{46 b)}$ of $\nu=0.445$. For the ring diblock copolymer the computer simulations $^{47)}$ give $\nu=0.45$. Please note that at high temperature the scaling in the melt of ring polymers should be the same as in the ring diblock copolymer system. The random phase approximation, with assumed Gaussian statistics, shows ${ }^{48,49)}$ that the transition to the ordered lamellar phase occurs at the temperature $T_{\text {ring }}=1.78 T_{\text {linear }}$ (for symmetric rings), where $T_{\text {linear }}$ is the temperature for the transition to the lamellar phase in the linear diblock copolymer system. This result compares very well with simulations ${ }^{47)}$, despite the fact that the ring statistics is certainly not Gaussian. When we decrease the temperature the rings strongly stretch along the axis connecting the centers of mass of two blocks. 
So far we have discussed the flexible-flexible diblock copolymer systems, where the diblock copolymers are modelled as Gaussian chains or, almost equivalently, chains with freely rotating bonds of fixed length. One can investigate the changes in the structure and stability of the disordered (isotropic) phase of the diblock copolymer system with the stiffness of a copolymer molecule. Microphase separated block copolymers with blocks of different stiffness are technologically important as composites. The flexible part of the polymer provides resistence to fracture while the rigid one resistence to tensile stresses and thermal stability. The simplest system of rigid-flexible diblock copolymers is discussed here ${ }^{50)}$. In a molecule one part of the copolymer is a chain with freely rotating bonds while the other is the rigid rod. In such a system, the density of monomers alone is not enough to specify the structure of the rod-rich domains nor the stability limits. In order to have a complete description of the system we must include a nematic order parameter tensor ${ }^{51)}$. In the high temperature isotropic phase, the various correlation functions between density and nematic order operators provide information about the structure. The correlation functions, in the Gaussian approximation, are found to oscillate with a characteristic period equal to the length of the rigid part. The lamellar domain boundaries of systems with longer rods are sharper than those with shorter ones, and the linear density measured along any direction is anticorrelated with the order parameter measuring nematic order in that direction. This latter effect is due to the isotropy of the system, and is relatively independent of the Maier-Saupe and Flory-Huggins parameters. This model is a special example of the more general model ${ }^{52)}$ shown in Fig.6. Here the A block consists of segments of small size $b_{A}$ and B block consisting of segments of much larger size $b_{B}$.

Liquid crystal polymers and their mixtures are studied both because of their practical utility and their intrinsic interest ${ }^{53,54)}$. In the case of mixtures ${ }^{55,56)}$, one 
wants to know how the location of various phases, isotropic and nematic, and their transitions depend on the properties of the two components, their rigidities, polymerization indices, interactions etc. An approach to this problem requires a model for the liquid crystal polymers. Recently, Mä̈ssa and Sixou ${ }^{57)}$ addressed these issues on by modelling the polymers as elastic lines with an energy of bending ${ }^{58)}$. Their calculation can be applied to many different mixtures, and they obtained various phase diagrams numerically for several values of the interaction constants. It is difficult, however, to extract general relationships between properties of the phase diagrams and the polymer parameters from their formalism. Such dependences can be made manifest if the Landau de Gennes expansion of the free energy is derived from the polymer model because the coefficients in this expansion depend upon the polymer characteristics of interest. Fredrickson and Leibler ${ }^{59)}$, inspired by experiments of Moore and Stupp ${ }^{56)}$, carried out this program in a model which also treated both polymers as elastic lines, ones characterized by different bending constants. Among other results, they found that the expansion was only applicable to this model if the two bending rigidities were large and nearly equal. On the other extreme there is a model of rigid flexible mixture where the differences in stiffness are large ${ }^{60)}$.

There are many phenomena which will not be discussed in this article. The mixtures are assumed as incompressible and compressiblity effects are ignored ${ }^{61)}$. The crossed-linked blends ${ }^{62)}$ and charged polymer systems ${ }^{63)}$, although interesting, will not be discussed here. We have concentrated in this paper on the equilibrium properties of various polymer systems; consequently the dynamics of blends ${ }^{64-66)}$ and diblock copolymer system ${ }^{67,68)}$ is not included.

The paper is organized as follows: In section II the mesoscopic model of polymer molecules is presented. Here we discuss the bond configuration for different 
systems, the various interaction hamiltonians steming from the Edwards model, density operators and order parameters and finally the conditional partition function. In section III we present the detailed study of the polymer blend system, including the discussion of the critical temperature in the Gaussian and one-loop approximation, the single chain statistics and the problem of the upper wavevector cutoff in polymer blends and in general in polymer systems. In section IV we discuss the diblock copolymer system. Here we discuss the behaviour of tagged chain in the melt of copolymers, phase diagram etc. The system is studied in the random phase approximation, one-loop approximation and self consistent field theory. In section $\mathrm{V}$ we study the random copolymer and multiblock copolymer systems. In section VI we present the results for the mixtures of homopolymers and copolymers. The correlations in the rigid-flexible diblock copolymer system are studied in section VII in the random phase approximation. The mixtures of rigid and flexible polymers are discussed in section VIII.

\section{The mesoscopic model of a polymer system}

The mesoscopic model of polymers is specified by the Hamiltonian describing the interaction between monomers, and the density distribution of monomers within a polymer molecule. Furthermore one has to specify the density operators which are needed to specify the structure of the system. The average of this operators is related to the order parameters. Next the conditional partition function has to be constructed. This is the partition function for the system subject to the constraint

of the the fixed configuration of the order parameter field. The logarithm of this partition function expanded in the order parameter gives the Landau-Ginzburg free energy. The calculations of correlations are also based on this partition function. Below we specify all the elements of the model. 


\section{A. Bond configuration}

The information that $\mathrm{N}$ monomers are connected and form a chain is specified by the distribution function $W[\mathbf{r}]$. To describe the flexible polymer, we use the chain model, in which atoms are described as being joined by freely rotating bonds of fixed length $l$. The distribution for $\mathrm{N}$ atoms in such a chain is given by ${ }^{69)}$

$$
W[\mathbf{r}]=\prod_{i=1}^{N} \frac{\delta\left(\left|\mathbf{u}_{i}\right|-l\right)}{4 \pi l^{2}}
$$

and is normalized as

$$
\int D \mathbf{r} W[\mathbf{r}]=1
$$

with

$$
D \mathbf{r}=\frac{1}{V} d \mathbf{r}_{0} d \mathbf{r}_{1} \cdots d \mathbf{r}_{N} .
$$

Here $\mathbf{u}_{i}=\mathbf{r}_{i}-\mathbf{r}_{i-1}$ is a vector specifying the orientation of the monomer and $r_{i}$ is the location of the $i$ point between the subsequent monomers. The same quantitative results at the level of the Landau-Ginzburg free energy are obtained if instead of the previous model we use the Gaussian model in the discrete version

$$
W[\mathbf{r}] \sim \exp \left(-\frac{3}{2 l^{2}} \sum_{i=1}^{N}\left|\mathbf{r}_{i}-\mathbf{r}_{i-1}\right|^{2}\right)
$$

or a continuous version of the model,

$$
W[\mathbf{r}] \sim \exp \left(-\frac{3}{2 l^{2}} \int_{0}^{N} d s\left|\frac{d \mathbf{r}(s)}{d s}\right|^{2}\right) .
$$

In both cases one normalizes the distribution.

The rigid polymers are described as rigid rods, or needles, in which all bonds have not only the same length, but also the same direction. In this case

$$
W[\mathbf{r}]=\frac{\delta\left(\left|\mathbf{u}_{1}\right|-l\right)}{4 \pi l^{2}} \prod_{j=2}^{N} \delta\left(\mathbf{u}_{j}-\mathbf{u}_{j-1}\right) .
$$


Finally, for a diblock copolymer in which a flexible chain consisting of $\mathrm{N}$ atoms has been joined with a rod containing $\mathrm{M}$ atoms,

$$
W[\mathbf{r}]=\prod_{i=1}^{N} \frac{\delta\left(\left|\mathbf{u}_{i}\right|-l\right)}{4 \pi l^{2}} \prod_{j=N+1}^{N+M} \delta\left(\mathbf{u}_{j}-\mathbf{u}_{j-1}\right)
$$

For semiflexible polymers one can use the model shown on Fig.6 which in the extreme limit reduces to the one discussed above. A different model of semiflexible polymers is the wormlike-chain model ${ }^{58,59)}$. In the discrete version we have:

$$
W[\mathbf{r}] \sim \exp \left(\frac{1}{2} k_{1} \sum_{i=1}^{N} \mathbf{u}_{i+1} \mathbf{u}_{i}\right),
$$

where $k_{1}$ is the bending elastic constant, which is the energy penalty for the change of angle between the nearests monomers. The continuous version of this model ${ }^{58)}$ is defined as follows:

$$
W[\mathbf{r}] \sim \exp \left(-\frac{1}{2} k_{1} \int_{0}^{N} d s\left|\frac{d \mathbf{u}(s)}{d s}\right|^{2}\right),
$$

subject to the constraint $|\mathbf{u}|^{2}=1$. The next step in the definition of the model is the specification of the interactions between the monomers.

\section{B. Interactions between monomers}

The typical length scale for a flexible chain is given by the radius of gyration i.e. the size of the region occupied by the chain in the melt. In the simplest Gaussian approximation one finds that it is proportional to $\sqrt{N} l$ and is much larger than the monomer size $l$. All the interesting phenomena take place at the length scale proportional to the radius of gyration. On the other hand the range of the potential is proportional to $l$, and therefore is not relevant to the phenomena discussed in the paper. Guided by this simple observation the following short range effective interaction potential has been proposed by Edwards ${ }^{70)}$ :

$$
v_{i j}^{\alpha \beta}\left(\mathbf{r}_{i}, \mathbf{r}_{j}\right)=w_{\alpha \beta} \delta\left(\mathbf{r}_{i}-\mathbf{r}_{j}\right)
$$


where $\delta$ is the Dirac delta function and $w_{\alpha \beta}$ is the effective interaction parameter for $\alpha$ and $\beta$ type monomers (e.g. A, B monomers in the binary homopolymer mixture). The effective interaction parameter is given by the integral ${ }^{70,71)}$ of the direct correlation function ${ }^{72,73)}$. In the first approximation for the rigid molecules, such integral, does not depend on temperature and is equal to the excluded volume, the volume inaccesible to one molecule when the other is fixed in space. In the case of two spheres of radius $D$ the excluded volume is the larger sphere of radius 2D. Apart from the repulsive forces there is also an attractive potential e.g. van der Waals potential. The direct correlation function is, in the first approximation proportional to this potential. Thus $w_{\alpha \beta}$ contains two contribution: one associated with the excluded volume and one with the attractive potential. Summarizing: the interactions given by Eq.(2.10) are related to the microscopic interaction potential via the direct correlation function. The delta function signifies the extreme short range character of the potential as measured in terms of the length given by the radius of gyration.

The interaction potential for rigid, elongated molecules, depends not only on the positions but also on the orientations of molecules. In the case of two rods ${ }^{51)}$ with fixed orientations the excluded volume has roughly the shape of a rectangular box. If we expand the excluded volume in terms of the Legendre polynomials of the cosine of the relative angle of two rods we obtain the constant term, proportionl to $w_{\alpha \beta}$, plus the second term related to the second Legendre polynomial, $P_{2}$. If higher order terms are neglected the anisotropic part of the potential is modelled $\mathrm{as}^{50,60)}$ :

$$
v_{i, j}^{\alpha \beta}\left(\mathbf{r}_{i}, \mathbf{u}_{i}, \mathbf{r}_{j}, \mathbf{u}_{j}\right)=v_{\alpha \beta} \delta\left(\mathbf{r}_{i}-\mathbf{r}_{j}\right) P_{2}\left(\frac{\mathbf{u}_{i} \mathbf{u}_{j}}{\left|\mathbf{u}_{i}\right|\left|\mathbf{u}_{j}\right|}\right)
$$

The total potential is given by the sum of $\mathrm{Eq}(2.10)$ and (2.11). Please note that in general the parameters $w_{\alpha \beta}$ and $v_{\alpha \beta}$ are not independent, since they follow from 
the same direct correlation function.

Finally for the dipolar monomers one can model the potential as:

$$
v_{i, j}^{\alpha \beta}\left(\mathbf{r}_{i}, \mathbf{u}_{i}, \mathbf{r}_{j}, \mathbf{u}_{j}\right)=v_{\alpha \beta} \delta\left(\mathbf{r}_{i}-\mathbf{r}_{j}\right)\left(\frac{\mathbf{u}_{i} \mathbf{u}_{j}}{\left|\mathbf{u}_{i}\right|\left|\mathbf{u}_{j}\right|}\right)
$$

The next step is the specification of the density operators needed for the definition of the order parameters.

\section{Density operators}

For the sake of clarity we shall discuss this point using the example of the rigidflexible diblock copolymer system ${ }^{50)}$. The chain architecture is given by $\operatorname{Eq}(2.6)$. The system consists of $n$ chains each consisting of $N_{A}$ A-type monomers joined to $N_{B}$, B-type monomers. The interactions between two monomers are described by the sum of $\operatorname{Eq}(2.10)$ and $\operatorname{Eq}(2.11)$. The density operators needed to specify the configuration of the system are as follows:

$$
\begin{gathered}
\hat{\phi}_{A}^{(n)}(\mathbf{r})=\frac{1}{\rho_{0}} \sum_{\gamma=1}^{n} \sum_{i=1}^{N_{A}} \delta\left(\mathbf{r}-\mathbf{r}_{i}^{\gamma}\right) ; \\
\hat{\phi}_{B}^{(n)}(\mathbf{r})=\frac{1}{\rho_{0}} \sum_{\gamma=1}^{n} \sum_{j=N_{A}+1}^{N_{A}+N_{B}} \delta\left(\mathbf{r}-\mathbf{r}_{j}^{\gamma}\right) ; \\
\hat{Q}_{\alpha \beta}^{(A)}(\mathbf{r})=\frac{1}{\rho_{0}} \sum_{\gamma=1}^{n} \sum_{i=1}^{N_{A}} \delta\left(\mathbf{r}-\mathbf{r}_{i}^{\gamma}\right)\left(\frac{3}{2} \frac{\left(\mathbf{u}_{i}^{\gamma}\right)_{\alpha} \cdot\left(\mathbf{u}_{i}^{\gamma}\right)_{\beta}}{\left|\mathbf{u}_{i}^{\gamma}\right|^{2}}-\frac{\delta_{\alpha \beta}}{2}\right) ; \\
\hat{Q}_{\alpha \beta}^{(B)}(\mathbf{r})=\frac{1}{\rho_{0}} \sum_{\gamma=1}^{n} \sum_{i=N_{A}+1}^{N_{A}+N_{B}} \delta\left(\mathbf{r}-\mathbf{r}_{i}^{\gamma}\right)\left(\frac{3}{2} \frac{\left(\mathbf{u}_{i}^{\gamma}\right)_{\alpha} \cdot\left(\mathbf{u}_{i}^{\gamma}\right)_{\beta}}{\left|\mathbf{u}_{i}^{\gamma}\right|^{2}}-\frac{\delta_{\alpha \beta}}{2}\right) .
\end{gathered}
$$

The first two operators, $\hat{\phi}_{A}^{(n)}(\mathbf{r})$ and $\hat{\phi}_{B}^{(n)}(\mathbf{r})$ (superscript (n) stands for the number of chains in the summation), represent the microscopic number fractions at point $\mathbf{r}$ of $\mathrm{A}$ and B monomers respectively; the next two tensor operators, $\hat{Q}_{\alpha \beta}^{(A)}(\mathbf{r})$ and $\hat{Q}_{\alpha \beta}^{(B)}(\mathbf{r})$ $(\alpha, \beta=1,2,3)$, represent the microscopic nematic tensor order parameters ${ }^{51)}$ at 
point $\mathbf{r}$ for the $\mathrm{A}$ and $\mathrm{B}$ monomers respectively. These tensors are symmetric and of zero trace, thus each has five independent components. The total number of independent operators is, therefore, twelve. Here $\rho_{0}$ is the average density of monomers in the system.

The total interaction hamiltonian, $\mathrm{H}$, is obtained by summing all the interactions between different monomers. Using the density operators it can be rewritten in the following simple form:

$$
\begin{gathered}
H=k_{B} T \rho_{0} \int d \mathbf{r}\left(\frac{1}{2} w_{A A}\left(\hat{\phi}_{A}^{(n)}\right)^{2}(\mathbf{r})+\frac{1}{2} w_{B B}\left(\hat{\phi}_{B}^{(n)}\right)^{2}(\mathbf{r})+w_{A B} \hat{\phi}_{A}^{(n)}(\mathbf{r}) \hat{\phi}_{B}^{(n)}(\mathbf{r})\right. \\
\left.-\frac{1}{3} v_{A A} \hat{Q}_{\alpha \beta}^{(A)}(\mathbf{r}) \hat{Q}_{\beta \alpha}^{(A)}(\mathbf{r})-\frac{1}{3} v_{B B} \hat{Q}_{\alpha \beta}^{(B)}(\mathbf{r}) \hat{Q}_{\beta \alpha}^{(B)}(\mathbf{r})-\frac{2}{3} v_{A B} \hat{Q}_{\alpha \beta}^{(A)}(\mathbf{r}) \hat{Q}_{\beta \alpha}^{(B)}(\mathbf{r})\right),
\end{gathered}
$$

where summation over repeated $\alpha$ and $\beta$ indices is implied.

There is no general recipe for the choice of the density operators. The hint as to the right choice is provided by the form of the interactions between monomers and the chain architecture. Here $\hat{\phi}_{\gamma}^{(n)}, \hat{Q}_{\alpha}^{(A)} \beta \gamma=A, B$ form a minimal set of order parameter operators necessary to describe the system in the Landau approach.

\section{Conditional partition function}

The conditional partition function, $Z\left[P_{i}\right]$ is the partition function for the system subject to the constraint that the microscopic operators, $\hat{P}_{i}$ be fixed at prescribed values, $P_{i}(\mathbf{r})$ i.e.

$$
Z\left[P_{i}\right]=\left\langle\prod_{i} \delta\left(\hat{P}_{i}-P_{i}\right)\right\rangle,
$$

where the average is calculated as follows:

$$
\langle\cdots\rangle=\frac{1}{Z_{0}} \prod_{\gamma=1}^{n} \int D \mathbf{r}^{\gamma} \cdots W\left[\mathbf{r}^{\gamma}\right] \exp \left(-H / k_{B} T\right)
$$

Here $k_{B} T$ is the Boltzman factor and $Z_{0}$ is the canonical partition function. The Landau-Ginzburg (LG) free energy (in the mean-field approximation), $\Omega\left[P_{i}\right]$, is 
given by

$$
\Omega\left[P_{i}\right]=-k_{B} T \ln Z\left[P_{i}\right] .
$$

The conditional partition function and the LG free enrgy are functionals of the order parameters $P_{i}$. The partition function of the system is given by the summation of the conditional partional function over all possible configurations of the fileds $P_{i}$ :

$$
Z=\prod_{i} \int D P_{i} Z\left[P_{i}\right] .
$$

In the following sections we shall apply this method to polymer systems.

\section{Binary mixture of flexible homopolymers}

We consider a mixture of $\mathrm{n}_{A}$ A-type polymers with $\mathrm{N}_{A}$ monomers in each molecule, and $\mathrm{n}_{B}$ B-type polymers with $\mathrm{N}_{B}$ monomers in each molecule inside a volume V. The anisotropic interactions, $v_{\alpha \beta}$ are neglected. For later convenience we introduce two fields,

$$
\Psi_{A}(\mathbf{r})=\phi_{A}^{\left(n_{A}\right)}(\mathbf{r})-\bar{\phi}
$$

and

$$
\Psi_{B}(\mathbf{r})=\phi_{B}^{\left(n_{B}\right)}(\mathbf{r})-(1-\bar{\phi}) .
$$

These fields describe the excess of the fraction of $\mathrm{A}$ or $\mathrm{B}$ monomers at the point $\mathbf{r}$

over their average values in the system, $\bar{\phi}=n_{A} N_{A} /\left(n_{A} N_{A}+n_{B} N_{B}\right)$, and $1-\bar{\phi}$; they are zero in the homogeneous system. Finally, we assume that the system is incompressible, i.e.

$$
\phi_{A}^{\left(n_{A}\right)}(\mathbf{r})+\phi_{B}^{\left(n_{B}\right)}(\mathbf{r})=1,
$$

so that

$$
\Psi_{A}(\mathbf{r})=-\Psi_{B}(\mathbf{r})=\Psi(\mathbf{r}) .
$$

For the Fourier transform of $\Psi, \Psi(\mathbf{q})=\int d \mathbf{r} \Psi(\mathbf{r}) e^{i \mathbf{r q}}$, it implies $\Psi(\mathbf{q}=0)=0$. Since the system is incompressible, only one interaction parameter is needed, namely the 
dimensionless Flory-Huggins parameter, $\chi, w_{A A}+w_{B B}-2 w_{A B}=-2 \chi<0$. In general $\chi$ is positive so that it is energetically favorable for the system to separate into A-rich and B-rich phases. The first aim of the study is the location of the critical point for the mixture, below which the system separates into A-rich and B-rich phases.

The field $\Psi$ is for the incompressible blend the only order parameter and consequently both the partition function and the LG free energy are the functionals of $\Psi$. Please note that $\Psi$ vanishes in the homogeneous system, but is nonzero at the coexistance curve. We find, using the results of the cumulant expansion given in Appendix $\mathrm{A}$ and $\mathrm{B}$, the following form of $\Omega[\Psi]$, for $\Psi$ independent on position:

$$
\begin{aligned}
\Omega[\Psi] & =\left(\frac{1}{2}\left(\frac{1}{N_{A} \bar{\phi}}+\frac{1}{N_{B}(1-\bar{\phi})}\right)-\chi\right) \Psi^{2} \\
& +\frac{1}{6}\left(\frac{1}{N_{B}(1-\bar{\phi})^{2}}-\frac{1}{N_{A} \phi^{2}}\right) \Psi^{3} \\
& +\frac{1}{12}\left(\frac{1}{N_{B}(1-\bar{\phi})^{3}}+\frac{1}{N_{A} \bar{\phi}^{3}}\right) \Psi^{4}
\end{aligned}
$$

Linear terms, in $\Psi$, have been omitted in Eq.(3.5) as they simply correspond to a shift, $h_{\Psi}$, in the exchange chemical potential $\mu=\left(\mu_{A} / N_{A}\right)-\left(\mu_{B} / N_{B}\right)$, i.e.

$$
h_{\Psi}=\mu-\frac{\ln \bar{\phi}}{N_{A}}+\frac{\ln (1-\bar{\phi})}{N_{B}}-\frac{1}{N_{A}}+\frac{1}{N_{B}}-\chi(1-2 \bar{\phi}) \text {. }
$$

Here $\mu_{A}, \mu_{B}$ are the chemical potentials of $A$ and $B$ monomers. Please note that $\mathrm{Eq}(3.5)$ can be also obtained (as it should) directly from the Flory-Huggins free energy by expanding it in $\Psi$. The critical point for the mixture corresponds to the vanishing of the first and second term in $\mathrm{Eq}(3.5)$, or in other words to the vanishing of the second and third derivative of the Flory-Huggins free energy with respect to the concentration. The location of the critical point in this mean-field approach (Appendix A and B) for the A,B polymer mixture is given by the well 
known equations:

$$
\chi_{c}^{m f}=\frac{\left(\sqrt{N_{A}}+\sqrt{N_{B}}\right)^{2}}{2 N_{A} N_{B}}
$$

and

$$
\bar{\phi}_{c}^{m f}=\frac{\sqrt{N_{B}}}{\sqrt{N_{A}}+\sqrt{N_{B}}},
$$

where $\chi_{c}^{m f}$ is the value of Flory-Huggins parameter at the critical point and $\bar{\phi}_{c}^{m f}$ is the critical concentration of A monomers ( $m f$ stands for mean-field). In order to include the fluctuations (i.e. dependence of $\Psi$ on positions) we have to study the correlations functions in the system. Please note that the $\chi$ parameter is rescaled by $k_{B} T$.

\section{A. Vertex functions and critical point}

First of all, the critical point is marked by a very strong scattering, i.e. the divergence of the scattering intensity $\mathrm{I}(\mathrm{q})$ at $q \rightarrow 0$. This quantity is proportional to

$$
I(q) \sim\left(\Gamma_{2}(\mathbf{q},-\mathbf{q})\right)^{-1}=\frac{1}{V}<\Psi(\mathbf{q}) \Psi(-\mathbf{q})>.
$$

Here the average is calculated according to the following formula:

$$
<\cdots>=\frac{1}{Z} \int D \Psi \cdots Z[\Psi]
$$

where $Z$ and $Z(\Psi)$ are defined in section II D.

At the critical point, $\Gamma_{2}$ goes to zero for $\mathbf{q} \rightarrow 0$. Additionally,

$$
\Gamma_{3}(\mathbf{q}, \mathbf{k},-\mathbf{q}-\mathbf{k})=V^{2} \frac{<\Psi(\mathbf{q}) \Psi(\mathbf{k}) \Psi(-\mathbf{q}-\mathbf{k})>}{<\Psi(\mathbf{q}) \Psi(-\mathbf{q})><\Psi(\mathbf{k}) \Psi(-\mathbf{k})><\Psi(\mathbf{q}+\mathbf{k}) \Psi(-\mathbf{q}-\mathbf{k})>}
$$

vanishes at the critical point for $\mathbf{q}, \mathbf{k} \rightarrow 0$. The two equations,

$$
\begin{gathered}
\Gamma_{2}(0,0)=0, \\
\Gamma_{3}(0,0,0)=0,
\end{gathered}
$$


locate the critical point in the $(T, \bar{\phi})$ plane.

The method dealing with the calculation of the averages in the form given by Eq.(3.9) is described in detail in Ref.[6,7]. Here we use an expansion in the number of loops (Chapter 6, Ref.[7]), which in the case of a polymer mixture has great benefit of being an expansion in a small parameter proportional to $1 / \sqrt{N}$. In the limit of $N \rightarrow \infty$, only the terms with no loops survive. The zeroth order approximation (no loops) corresponds to the random phase approximation ${ }^{2)}$ (see Appendix A and B, Eq.(B.11) and the critical point location is given by Eqs.(3.7-8), thus it is the same result as the Flory-Huggins result.

The equations for the vertex functions in the one-loop approximation are discussed in Appendix $\mathrm{C}$ and Fig.7 and Fig.8 show the one loop diagrams which contribute to the vertex functions. Now we can proceed with the solution of Eqs. $(3.11,12)$ in the one-loop approximation (Appendix C). We are interested only in the first corrections to the mean-field results. Thus, we assume the solution in the following form:

$$
\chi_{c}=\chi_{c}^{m f}\left(1+\alpha_{1}\right),
$$

and

$$
\bar{\phi}_{c}=\bar{\phi}_{c}^{m f}\left(1+\alpha_{2}\right),
$$

where $\alpha_{1}$ and $\alpha_{2}$ are small corrections of the order $1 / \sqrt{N}$ and $\chi_{c}^{m f}$ and $\bar{\phi}_{c}^{m f}$ are given by Eqs.(3.7-8).In order to simplify the lengthy formulas, we also assume that the lengths of the monomers are equal, $l_{A}=l_{B}=l$, but, of course, it is straighforward to perform the calculations without this assumption. Combining all these equations 
we find $\chi_{c}$ and $\bar{\phi}_{c}$ in the following form:

$$
\begin{gathered}
2 \chi_{c}=\frac{\left(\sqrt{N_{A}}+\sqrt{N_{B}}\right)^{2}}{N_{A} N_{B}}\left(1+\left(\frac{12}{\pi \rho_{0} l^{3}}\right)\left(\frac{l}{\Lambda}\right)+\left(\frac{4 \pi}{3 \rho_{0} l^{3}}\right)\right. \\
\left.\times\left(\frac{N_{A}^{3 / 2}+N_{B}^{3 / 2}}{\sqrt{N_{A}}+\sqrt{N_{B}}}\right)\left(\frac{l}{\Lambda}\right)^{3}-\left(\frac{2 \pi}{3 \rho_{0} l^{3}}\right)\left(\sqrt{N_{A}}-\sqrt{N_{B}}\right)^{2}\left(\frac{l}{\Lambda}\right)^{3}\right), \\
\bar{\phi}_{c}=\frac{\sqrt{N_{B}}}{\sqrt{N_{A}}+\sqrt{N_{B}}}\left(1+\left(\frac{4 \pi}{\rho_{0} l^{3}}\right) \frac{\left(\sqrt{N_{A}}-\sqrt{N_{B}}\right)^{3}}{\sqrt{N_{B}}}\left(\frac{l}{\Lambda}\right)^{3}\right. \\
+\left(\frac{36}{\pi \rho_{0} l^{3}}\right) \frac{\left(\sqrt{N_{A}}-\sqrt{N_{B}}\right) \sqrt{N_{A}}\left(\frac{l}{\Lambda}\right)}{\left(\sqrt{N_{A}}+\sqrt{N_{B}}\right)^{2}} \\
\left.+\left(\frac{4 \pi}{\rho_{0} l^{3}}\right) \frac{\left(N_{A}^{3 / 2}-N_{B}^{3 / 2}\right) \sqrt{N_{A}}}{\left(\sqrt{N_{A}}+\sqrt{N_{B}}\right)^{2}}\left(\frac{l}{\Lambda}\right)^{3}\right)
\end{gathered}
$$

where $\Lambda$ is the cutoff discussed in Appendix D.

For $N_{A}=N_{B}=N$, we have $\bar{\phi}_{c}=1 / 2$ and

$$
\begin{aligned}
\chi_{c} & =\frac{2}{N}\left(1+\left(\frac{12}{\rho_{0} l^{3} \pi C_{0} \sqrt{N}}\right)\right. \\
& \left.+\left(\frac{4 \pi}{3 \rho_{0} l^{3} C_{0}^{3} \sqrt{N}}\right)\right),
\end{aligned}
$$

where $C_{0}=\sqrt{C_{1}+C_{2}}$ is a constant (see Appendix D). Assuming $C_{0}=1$ and the volume fraction (the ratio of the volume occupied by monomers to the total volume) $\rho_{0} l^{3}=1$ we find

$$
\chi_{c}=\frac{2}{N}\left(1+\left(\frac{8}{\sqrt{N}}\right)\right) .
$$

We can use this formula to estimate the shift of the critical temperature induced by fluctuations. Let $T_{c}^{m f}=400 \mathrm{~K}$ for $N=10^{3}$. Then we find from Eq.(3.18) that $T_{c}=319 \mathrm{~K}$. The shift, $\Delta T=T_{c}^{m f}-T_{c}$ is 81 degrees! Even for $N=10^{4}$ we have still $8 \%$ correction to $\chi_{c} / \chi_{c}^{m f}$. Of course, the constant $C_{0}$ should be the fitting parameter in the analysis of experimental results and its value can be larger or smaller than one. Nonetheless, we think that it should be of the order of 1 . 
For $N_{A}>N_{B}$, we find that $\bar{\phi}_{c}$ is larger than the mean-field value of the concentration of A-monomers, $\bar{\phi}_{c}^{m f}$. For $N_{A} \gg N_{B}$, it follows that

$$
\bar{\phi}_{c} \approx \sqrt{\frac{N_{B}}{N_{A}}}\left(1+\left(\frac{4 \pi}{\rho_{0} l^{3} C_{1}^{3 / 2}}\right)\left(\frac{1}{\sqrt{N_{B}}}\right)\right) .
$$

For $N_{A} \rightarrow \infty$ and $N_{B}$ finite, the critical concentration approaches zero, while the critical temperature approaches $T_{c}^{m f}$.

Finally, let us discuss the order of the n-loop diagram in the limit of $N_{A} \gg N_{B}$. In this limit $\bar{\phi}_{c} \approx \sqrt{N_{B}} / \sqrt{N_{A}}$ and is very small. It enters the n-loop diagram as a multiplicative constant in the form $1 / \bar{\phi}^{n+1}$. Additionally, we have $1 / q^{2}$ contributions for $n-1$ loops in the n-loop diagram. After grouping all these terms and using the formulas for the cutoff (Appendix D), we find that any n-loop diagram is proportional to $1 /\left(\sqrt{N_{A}} N_{B}^{(n+1) / 2}\right)$, and it follows that the small parameter in the loop expansion is now $1 / \sqrt{N_{B}}$. Because of the $1 / \sqrt{N_{A}}$ factor we also find that in the limit of $N_{A} \rightarrow \infty T_{c} \rightarrow T_{c}^{m f}$ even for finite $N_{B}$. This result is in accordance with de Gennes' predictions ${ }^{1)}$, for the polymer in a solvent. As we can see, the loop expansion breaks down if either $N_{A}$ or $N_{B}$ is of the order of unity, since then it is no longer true that the succesive n-loop terms are small in comparison with the (n-1)-loop terms. This analysis also prove that the loop expansion in polymer blends is indeed the expansion in small parameter, providing we chose the cutoff according to the prescription given in Appendix D.

\section{B. The Ginzburg criterion}

The critical temperature and concentration are the nonuniversal quantities depending on the details of the system. On the other hand, the values of the critical exponents are universal and do not depend on any microscopic details of the system. A set of critical exponents characterizes the whole class of critical points. For example the critical exponents for the Ising spin system, low-molecular mass bi- 
nary mixtures and polymer binary mixtures are the same. All these critical points are said to belong to the Ising universality $\operatorname{class}^{7)}$. As was mentioned in the Introduction, the scattering intensity diverges on the approach to the critical point, according to the following formula: $I(q \rightarrow 0) \sim\left(T / T_{c}-1\right)^{-\gamma}$. We know that for the Ising universality class $\gamma=1.26$, while in the mean-field theory we get $\gamma=1$. As we have seen in the previous section the fluctuations shift the critical temperature, however the most important effect of fluctuations is the change of the value of the critical exponents. In the experimental analysis ${ }^{8-13)}$ of $I(q)$, in order to extract $\gamma$, one plots $\log I(q)$ versus $\log \left(T / T_{c}-1\right)$. (see Fig.9). It has been observed that away from the critical point the plot is a straight line with the slope of 45 degrees, indicating $\gamma=1$. As one approaches $T_{c}$ closer the straight line begins to curve. Finally, very close to the critical point it becomes a straight line again, but with the slope corresponding to $\gamma=1.26$. The change in the slope marks the breakdown of the mean-field theory. The criterion that tells us when this happens is called the Ginzburg criterion. The region around the critical point where $\gamma \neq 1$ is called the Ginzburg region. Since $I^{-1}(q) \sim \Gamma_{2}(q,-q)$, we can base our estimate of the Ginzburg region on the analysis of the two-body vertex function given by Eq.(3.9). Our strategy is to write it down as a sum of two terms: a mean-field one and a correction to it. As we approach the critical point the corrections grow in comparison to the mean-field term. Finally they are larger than the mean-field term, indicating the breakdown of the mean-field theory. This happens inside the Ginzburg region.

Close to the critical point and in the limit of $q, p \rightarrow 0$ we can rewrite Eq.(3.9) and Eq.(3.10) in the following form:

$$
\Gamma_{2}(0,0)=A_{1}\left(\bar{\phi}-\bar{\phi}_{c}^{m f}\right)^{2}+2\left(\chi_{c}^{m f}-\chi\right)+F_{1}(\bar{\phi}, \chi)
$$


and

$$
\Gamma_{3}(0,0,0)=A_{1}\left(\bar{\phi}-\bar{\phi}_{c}^{m f}\right)+F_{2}(\bar{\phi}, \chi)
$$

where $A_{1}=2\left(1 / N_{A}\left(\bar{\phi}_{c}^{m f}\right)^{3}+1 / N_{B}\left(1-\bar{\phi}_{c}^{m f}\right)^{3}\right)$ and $F_{1}$ and $F_{2}$ are the one-loop corrections to $\Gamma_{2}(0,0)$ and $\Gamma_{3}(0,0,0)$, respectively. They have been discussed in detail in the Appendix C. Now we can express $\bar{\phi}_{c}^{m f}$ and $\chi_{c}^{m f}$ in terms of the critical concentration $\bar{\phi}_{c}$ and the Flory-Huggins parameter at the critical point $\chi_{c}$. Now Eq.(3.20) reads as follows:

$\Gamma_{2}(0,0)=A_{1}\left(\bar{\phi}-\bar{\phi}_{c}\right)^{2}+2\left(\chi_{c}-\chi\right)+\left(F_{1}(\bar{\phi}, \chi)-F_{1}\left(\bar{\phi}_{c}, \chi_{c}\right)\right)-2 F_{2}\left(\bar{\phi}_{c}, \chi_{c}\right)\left(\bar{\phi}-\bar{\phi}_{c}\right)$.

This equation is the basis for the quantitative formulation of the Ginzburg criterion, namely, the mean-field theory is valid providing the first two terms in Eq.(3.22) (giving the mean field result for $\gamma$ ) are much larger than the last two terms, which are the corrections. Thus the mean-field theory breaks down when

$$
A_{1}\left(\bar{\phi}-\bar{\phi}_{c}\right)^{2}+2\left(\chi_{c}-\chi\right) \leq-\left(F_{1}(\bar{\phi}, \chi)-F_{1}\left(\bar{\phi}_{c}, \chi_{c}\right)\right)+2 F_{2}\left(\bar{\phi}_{c}, \chi_{c}\right)\left(\bar{\phi}-\bar{\phi}_{c}\right)
$$

Now, we can give the explicit formula for the Ginzburg criterion in the special case of $N_{A} \approx N_{B}$ and $\bar{\phi} \approx \bar{\phi}_{c}$. Neglecting the dependence of $\Gamma_{4}^{(0)}$ on $\mathbf{k}$, i.e. assuming the approximation $\Gamma_{4}^{(0)}(\mathbf{k},-\mathbf{k}, 0,0) \approx \Gamma_{4}^{(0)}(0,0,0,0)$ we find the Ginzburg criterion in following form:

$$
2\left(\chi_{c}-\chi\right)+A_{1}\left(\bar{\phi}-\bar{\phi}_{c}\right)^{2}=\frac{C_{g}\left(N_{A}^{-1} \bar{\phi}_{c}^{-3}+N_{B}^{-1}\left(1-\bar{\phi}_{c}\right)^{-3}\right)^{2}}{\rho_{0}^{2}\left(R_{A}^{2} N_{A} \bar{\phi}_{c}^{-1}+R_{B}^{2} N_{B}\left(1-\bar{\phi}_{c}\right)^{-1}\right)^{3}}
$$

Here $C_{g}$ is a constant, $R_{i}^{2}=N_{i} l^{2} / 6(i=A, B)$. For $\bar{\phi}=\bar{\phi}_{c}$, Eq.(3.24) reduces to Eq.(3) of Ref[10] providing we change $v_{m}$ to $1 / \rho_{0}, \bar{\phi}_{c}^{m f}$ to $\bar{\phi}_{c}, \chi_{c}^{m f}$ to $\chi_{c}$ and $C$ to $C_{g} / 2$. On Fig.10 we show the plot of the scattering intensity versus the inverse 
of the temperature ${ }^{10)}$. Please note that the mean field critical temperature is not correctly identified on this $\operatorname{plot}^{15)}\left(\right.$ see also $\left.{ }^{74)}\right)$, since we find that

$$
I(q \rightarrow 0) \sim\left(\chi_{c}-\chi\right)^{-1}
$$

where $\chi_{c}$ is not the mean-field Flory Huggins critical parameter. In fact the Ginzburg region is of the order of $1 / N$ whereas the difference between the critical temperature and the its mean field value goes as $1 / \sqrt{N}$. Therefore it is not correct to identify the mean field critical temperature with the point where the extrapolated straight line representing $I(q \rightarrow 0)$ far from the critical point crosses the temperature axis.

Finally, we consider the limiting case of $N_{A} \gg N_{B}$. In the first approximation and for $\bar{\phi}=\bar{\phi}_{c}$, one finds that the mean-field theory breaks down for

$$
\frac{\left(\chi_{c}-\chi\right)}{\chi_{c}} \sim \frac{1}{\sqrt{N_{A} N_{B}}} .
$$

Thus, for $N_{A} \rightarrow \infty$, one would expect that the mean-field theory is valid in the whole region around the critical point, for any value of $N_{B}$ (Note that $1 / \sqrt{N_{A}}$ term here comes from the $1 / \sqrt{N_{A}}$ term which apppears in the loop expansion). This result is misleading for the following reasons: In our analysis we have not specified the temperature below which we can expect any influence of the critical point on the behavior of the polymer mixture. Such a temperature is called the theta temperature and is denoted, $\Theta$. Above this temperature mixing is strongly favored and the influence of the critical point on the scattering intensity is negligible. Only below $\Theta$ can we expect any sign of critical behavior (either mean-field or Ising-like). This means that if the Ginzburg region estimated above is larger than the region between $\Theta$ and $T_{c}$ we will never see the mean-field behavior, characterized by $\gamma=1$, but only the Ising-like behavior, characterized by $\gamma=1.26$. As shown by Joanny ${ }^{5)}$

$$
\frac{T-T_{c}}{\Theta-T_{c}} \approx \frac{1}{\sqrt{N_{B}}}
$$


marks the breakdown of the mean-field theory in the aforementioned sense. Finally we see that in the limit of $N_{B} \rightarrow 1$ the mean-field theory breaks down completely even for infinite $N_{A}$. As mentioned in the previous section also the loop expansion breaks down, but peculiarly the critical temperature approaches its mean-field value.

\section{Single chain statistics}

The single chain statistics is determined in the first place by the correlation function for the single chain. In the A,B homopolymer mixture we chose one chain (e.g. A) and tag it by coupling it to the fictitious field $U_{A}$. The correlation function is given by ${ }^{17)}$ :

$$
S_{A A}\left(\mathbf{q}_{1}, \mathbf{q}_{2}\right)=<\hat{\phi}_{A}^{(1)}\left(\mathbf{q}_{1}\right) \phi_{A}^{(1)}\left(\mathbf{q}_{2}\right)>=\frac{\delta^{2} Z\left[U_{A}\right]}{Z\left[U_{A}\right] \delta U_{A}\left(\mathbf{q}_{1}\right) \delta U_{A}\left(\mathbf{q}_{2}\right)} .
$$

where the partition function (section II) $Z$ as a functional of the external field $U_{A}$ is given by

$$
\begin{aligned}
Z\left[U_{A}\right]= & \int D \phi_{A}^{\left(n_{A}\right)} \int D \phi_{B}^{\left(n_{B}\right)} \exp \left(-H_{I}\left[\phi_{A}^{\left(n_{A}\right)}, \phi_{B}^{\left(n_{B}\right)}\right]\right) \\
& \int D J_{A} \int D J_{B} \exp \left(i \int \frac{d \mathbf{q}}{(2 \pi)^{3}} \phi_{A}^{\left(n_{A}\right)} J_{A}+i \int \frac{d \mathbf{q}}{(2 \pi)^{3}} \phi_{B}^{\left(n_{B}\right)} J_{B}\right. \\
+ & \left.F_{A}^{\left(n_{A}\right)}\left[J_{A}\right]+F_{A}^{(1)}\left[J_{A}+i U_{A}\right]-F^{(1)}\left[J_{A}\right]+F_{B}^{\left(n_{B}\right)}\left[J_{B}\right]\right) .
\end{aligned}
$$

Here $F_{\gamma}^{\left(n_{\gamma}\right)}$ is defined in section II and Appendix A. Now using this equation at $U_{A}=0$ we find the following approximate equation for the single chain structure 
factor in the incompressible blend:

$$
\begin{aligned}
& V S_{A A}(\mathbf{q},-\mathbf{q})=<\hat{\phi}_{A}^{(1)}(\mathbf{q}) \hat{\phi}_{A}^{(1)}(-\mathbf{q})>_{0}+\frac{1}{2 \rho_{0}} \int \frac{d \mathbf{k}}{(2 \pi)^{3}} \\
& \left(<\hat{\phi}_{A}^{(1)}(\mathbf{q}) \hat{\phi}_{A}^{(1)}(-\mathbf{q}) \hat{\phi}_{A}^{(1)}(\mathbf{k}) \hat{\phi}_{A}^{(1)}(-\mathbf{k})>_{0}\right. \\
& \left.-<\hat{\phi}_{A}^{(1)}(\mathbf{q}) \hat{\phi}_{A}^{(1)}(-\mathbf{q})>_{0}<\hat{\phi}_{A}^{(1)}(\mathbf{k}) \hat{\phi}_{A}^{(1)}(-\mathbf{k})>_{0}\right) \\
& \frac{\Gamma_{2}^{(A)}(\mathbf{k}) \Gamma_{2}^{(A)}(\mathbf{k})}{\Gamma_{2}(\mathbf{k})}
\end{aligned}
$$

It includes the ideal term and the first nonvanishing correction to it. Here $\Gamma_{2}$ is defined in Appendix $\mathrm{C}$ and $\Gamma_{2}^{(A)}$ in Appendix A. As defined in the Appendix A the averages with the subscript 0 are taken over the configurations of noninteracting system (ideal averages) whereas those without the subscript are taken over the configurations of the interacting system.

The equation for the single chain correlation function in $\operatorname{Ref}[20]$ is similar in structure to $\mathrm{Eq}(3.30)$, only instead of having $\Gamma_{2}^{(A)}(\mathbf{k}) \Gamma_{2}^{(A)}(\mathbf{k}) \Gamma_{2}^{-1}(\mathbf{k})$ one has there $<J_{A}(\mathbf{k}) J_{A}(-\mathbf{k})>$. The latter is exactly equal to $\chi\left(1+2 \rho_{0} \chi \Gamma_{2}^{-1}(\mathbf{k})\right) / 2$. Note that if we use the linear approximate relation between $J_{A}$ and $\Psi_{A}$ i.e. $J_{A}=\Gamma_{2}^{(A)} \Psi_{A}$ we would get the same result from both approaches. This equation for the single chain correlation function should be supplemented by the self-consistent equations for the collective structure factor, $\Gamma_{2}(\mathbf{k})$ (Appendix $\mathrm{C}$ ). The equation for the single chain correlation function and the collective vertex functions are also coupled via the radius of gyration (see Appendix D) given by ${ }^{75)}$ :

$$
R_{A}^{2}=\frac{1}{2 N_{A}^{2}}<\sum_{i=1}^{N_{A}} \sum_{j=1}^{N_{A}}\left(\mathbf{r}_{i}^{(1)}-\mathbf{r}_{j}^{(1)}\right)^{2}>
$$

It is obtained from $\mathrm{Eq}(3.30)$ by differentiating both sides twice with respect to $q$ and taking the limit of $q \rightarrow 0$. We find

$$
2 N_{A}^{2} R^{2} / 3=2 N_{A}^{2} R_{0}^{2} / 3-\frac{1}{2 \rho_{0}} \int \frac{d \mathbf{k}}{(2 \pi)^{3}} f(\mathbf{k}) \Gamma_{2}^{(A)}(\mathbf{k}) \Gamma_{2}^{(A)}(\mathbf{k}) \Gamma_{2}^{-1}(\mathbf{k})
$$


where $R_{0}^{2}=N_{A} l^{2} / 6$ is the radius of gyration for the ideal chain $(\mathrm{Eq}(3.3)$ with the

ideal average) and $f(\mathbf{k})$ is explicitely given in Appendix E. Since $f(\mathbf{k})$ is always positive we find the first intuitively obvious result that chains in a blend shrink below the Gaussian chain limit.

The role of the cutoff (Appendix D) is also clear here. If not for the $\sqrt{N}$ in the upper wavevector cutoff the correction to the Gaussian radius of gyration would be nonzero even in the limit of $N \rightarrow \infty$ and thus RPA would not be a correct description in this limit.

\section{Linear flexible diblock copolymer system}

An AB diblock copolymer molecule consists of two blocks (A and B) joint together. The blocks are incompatible, but contrary to the case of AB homopolymer blend cannot separate on the macroscopic scale due to the chemical bond joining them. Nonetheless as we lower the temperature the blocks separate on the scale of the radius of gyration forming the periodic structures - the phase transition between the disordered phase and ordered is called, in the polymer community jargon, the microphase separation. In the case of the diblock copolymer the concentration of A monomers is set by the architecture of the chain, the average fraction of A monomers is given by

$$
f=\frac{N_{A}}{N}
$$

where $N=N_{A}+N_{B}$ is the total number of monomers in the diblock copolymer molecule. The order parameter field,

$$
\Psi(\mathbf{r})=\phi_{A}^{(n)}(\mathbf{r})-f
$$

is defined as in section II and $n$ is the number of copolymers. Incompressibility constraint is implied. Contrary to the case of homopolymer blends the equilibrium 
distribution of $\mathrm{A}$ monomers at low temperatures, given by $\Psi$, is not uniform in space.

\section{A. Scattering intensity in the random phase approximation}

Leibler $^{22)}$ applied random phase approximation (RPA) scheme (described in Appendix A and B, see also Ref[76,20]) to the system and found the following form of the two body correlation function $(\mathrm{Eq}(3.9))$ :

$$
I(q)=\frac{W_{1}(q)}{W_{2}(q)-2 \rho_{0} \chi W_{1}(q)}
$$

where $W_{1}(q)=S_{11} S_{22}-S_{12}^{2}$ and $W_{2}(q)=S_{11}+S_{22}+2 S_{12}$ depend only on the architecture of the diblock copolymer (see section II); $W_{1}$ is the determinat and $W_{2}$ the sum of all the elements of the matrix $S_{\alpha \beta}$, where

$$
\begin{gathered}
S_{11}=\frac{1}{V}<\phi_{A}^{(n)}(q) \phi_{A}^{(n)}(-q)>_{0}=\frac{N}{\rho_{0}} g_{1}(f, x), \\
S_{22}=\frac{1}{V}<\phi_{B}^{(n)}(q) \phi_{B}^{(n)}(-q)>_{0}=\frac{N}{\rho_{0}} g_{1}(1-f, x)
\end{gathered}
$$

and

$$
S_{12}=S_{21}=\frac{1}{V}<\phi_{A}^{(n)}(q) \phi_{B}^{(n)}(-q)>_{0}=\frac{N}{2 \rho_{0}}\left(g_{1}(1, x)-g_{1}(f, x)-g_{1}(1-f, x)\right)
$$

Here $x=q^{2} N l^{2} / 6, \mathrm{~V}$ is the volume and

$$
g_{1}(f, x)=2(f x+\exp (-f x)-1) / x^{2}
$$

Please note (compare with Appendix A) that since the blocks A and B are joined the cross corelation function $S_{12}$ is non zero here. The behavior of $I(q)$ is shown in Fig.11. As we increase $\chi$ (lower the temperature) the peak of $I(q)$ grows and finally diverges at the well defined (and fixed) $q^{*}$ vector. Interestingly the position of the peak in this approach is fixed independent of the temperature. For $\mathrm{f}=1 / 2$ 
$I(q)$ diverges at $\chi N=10.55$, while for $f=0.25$ at $\chi N=17.6$. The divergence of the scattering intensity indicates the onset of ordering at the typical length scale given by the inverse of the scattering vector $q^{*}$.

The order parameter depends on position in the spatially ordered phase. The free energy in the random phase approximation has the following form in this case:

$$
\Omega[\Psi]=\sum_{n=1}^{\infty} \int \frac{d \mathbf{q}_{1}}{(2 \pi)^{3}} \cdots \frac{d \mathbf{q}_{n}}{(2 \pi)^{3}} \Gamma_{n}^{(0)}\left(\mathbf{q}_{1} \cdots \mathbf{q}_{n}\right) \Psi\left(\mathbf{q}_{1}\right) \cdots \Psi\left(\mathbf{q}_{n}\right)
$$

where the vertex functions $\Gamma_{n}^{(0)}$ are given in $\operatorname{Ref}[20,76,22]$ and the general method of the calculation has been described in Appendix A and B. The equilibrium configuration of the system corresponds to the minimum of $\Omega$ i.e. to the solution of the functional equation:

$$
\frac{\delta \Omega[\Psi]}{\delta \Psi}=0
$$

In practice this complicated equation is simplified if we assume a given form (in accordance with the symmetry of the phase) of the spatial dependence of $\Psi(\mathbf{r})$ in the ordered phase. For example in the lamellar phase:

$$
\Psi(\mathbf{r})=A \cos \left(q_{l} z\right)
$$

Now inserting (4.10) into $\mathrm{Eq}(4.8)$, we find the free energy in the lamellar phase as a function of two variables, the amplitude, $A$ and the wavevectors $q_{l}$. The period of the lamellar phase is equal to $2 \pi / q_{l}$. Now the minimum of the free energy in the lamellar phase corresponds to the solution of two algebraic equations:

$$
\frac{\partial \Omega\left(A, q_{l}\right)}{\partial A}=0
$$

and

$$
\frac{\partial \Omega\left(A, q_{l}\right)}{\partial q_{l}}=0
$$


The phase transition between the disordered phase and the lamellar phase takes place at the point where both energies are equal. In the random phase approximation the transition is second order and takes place for $q_{l}=q^{*}$ and at the point where the scattering intensity diverges. Apart from the lamellar phase one studies in the same way ${ }^{22)}$, other phases such as hexagonal phase, bcc phase, obdd phase or others. For example the simplest approximation of $\Psi$ in the hexagonal phase has the following form:

$$
\Psi(\mathbf{r})=A \sum_{n=1}^{3} \cos \left(\mathbf{q}_{n} \mathbf{r}_{\perp}\right),
$$

where $q_{1}=(1,1 / \sqrt{3}) q_{h}, q_{2}=(-1,1 / \sqrt{3}) q_{h}$ and $q_{3}=(0,2 / \sqrt{3}) q_{h}$ are the vectors spanning the first shell (similarly in the lamellar phase $\mathrm{Eq}(4.10)$ ) in the reciprocal space for the regular hexagonal lattice. The form of $\Psi$ given by $\operatorname{Eq}(4.10)$ for the lamellar phase and $\mathrm{Eq}(4.13)$ for the hexagonal phase is a good approximation close to the weakly first order or second order phase transitions, it breaks down at low temperatures, when the ordered domains are sharp ${ }^{76)}$.

In the random phase approximation the hexagonal phase and bcc phases are also stable in some range of parameters. The transitions between these phases and disordered phase are first order. The phase diagram of the diblock copolymer system is shown in Fig.12. In the weak segregation regime the random phase approximation is exact in the limit of $N \rightarrow \infty$.

\section{B. Fluctuations induced first order phase transition}

The transition to the lamellar phase from the disordered phase is second order in the random phase approximation. It can be easily seen if we use Eq(4.10), apply it to $\operatorname{Eq}(4.8)$ and expand the free energy in the amplitude $A$. We find the following form of $\Omega$ (keeping the first three terms only):

$$
\Omega=a A^{2}+b A^{4}+c A^{6}
$$


Here $b$ and $c$ are two positive constants independent of the temperature and $a$ changes sign at the point when the scattering intensity $I(q)(\mathrm{Eq}(4.3))$ diverges. At this point we have the second order phase transition. Now if we go beyond the random phase approximation and include the one-loop corrections the second term in $\mathrm{Eq}(4.14)$ becomes negative before $a$ does, but $c$ remains positive and consequently the transition becomes first order ${ }^{20,23,77)}$. The free energy in the one loop approximation has the same general form given by $\mathrm{Eq}(4.8)$, but the coefficients in the expansion are given by the vertex functions $\Gamma_{n}$ calculated in the one loop approximation. The equation for the two and three body vertex function in the one-loop approximation is given in Appendix D. These equations are general and valid for polymer blend system as well as for the disordered phase of diblock copolymers. In the ordered lamellar phase the equation for vertex function are slightly different, since the field $\Psi$ given by $\operatorname{Eq}(4.10)$ is non-uniform. In the case of $f=1 / 2 \Gamma_{3}=0$ due to the symmetry ${ }^{22)}$ and the one-loop equation for the lamellar phase has the form given in Appendix $\mathrm{F}^{20}$ ). The phase diagram in the one-loop aproximation is shown in Fig.13. As we can see the transition to the lamellar phase occurs not only for the symetric case $f=1 / 2$ (as we have found in the random phase approximation), but also in the neighbourhood of this point. The transition to the lamellar phase is first order as mentioned previously. In the limit of $N \rightarrow \infty$ the one-loop corrections vanish and the results reduce to the random phase approximation.

In the random phase approximation the peak in the scattering intensity is fixed and scales with the polimerization index as $\sqrt{N}$. However from the experiments ${ }^{78)}$ it follows that the peak in the structure factor exibits a much stronger dependence on $N$ than predicted by RPA. In Fig.14 the comparison of the results of the one-loop approximation $^{20)}$ and experiment are shown. The logarithm of the peak position is plotted against the logarithm of $\mathrm{N}$. The agreement between the theory and ex- 
periment is remarkable, especially that no adjustable parameters has been used. The vertical straight line is the predicted $\mathrm{N}$ for which the microphase separation occurs (the temperature is fixed here at $\mathrm{T}=296 \mathrm{~K}$ ). Close to this transition the peak position $q^{*}$ is approximately represented by $N^{-0.8}$.

Since the peak position deviates strongly from the $\sqrt{N}$ behavior, it indicates that the conformation of a single polymer molecule should also differ from the Gaussian behavior. Indeed, both in the theory ${ }^{20)}$ and computer simulations ${ }^{21)}$, strong deviation from the Gaussian behavior has been found. The equations for the radius of gyration for the polymer blend are discussed in section III, the very similar equations are found for the diblocks ${ }^{20)}$. The solution of these equations indicate that the chains strongly stretch as the temperature is lowered towards the microphase separation transition temperature. The configuration of the chains indicates that stretching occurs at the point where the two blocks are joined. However the blocks themselves shrink below the gaussian limit. Thus the configuration of the chain resembles the dumbell, consisting of two coils more densely packed than the Gaussian coils, and stretched above the Gaussian limit. The fact that the coils shrink below the Gaussian limit has the same origin as the shrinking of the polymer chains in the blends close to the critical point (section III C). Interestingly the deviations from the Gaussian behavior occur far from the microphase separation temperature, deep in the disordered phase.

\section{Gyroid phase and self consistent field theory}

The diblock copolymer system forms a variety of phases. So far we have only mentioned the simplest structures studied in the theory: the lamellar, hexagonal and body center cubic structures. Apart from those simple morphologies the diblock copolymer system exhibit new type of structures: the bicontinous phases where the junction between the blocks are located at the periodic continuous internal 
interfaces. The double diamond and gyroid structures are shown in Fig.1 and 2 . Both were observed in the polystyrene-polisoprene system. The first one in the strong segregation regime ${ }^{30-32}$ ) (which is not discussed in this paper), while the second one in the weak segregation regime ${ }^{79)}$, where the Landau theory should be valid. This phase has not been studied in the random phase approximation, but in the mean-field theory called the self consistent field theory ${ }^{24)}$. The latter theory applied to the system of diblock copolymers predicts the stable gyroid phase between the lamellar and hexagonal phase as shown on Fig.15. In this theory the problem of computing the partition function for $\mathrm{n}$ chains is reduced to the single chain in the external field. The difference between this approach and the random phase approximation starts at the level of the partition function for the system of noninteracting chains in the external fields $J_{A}, J_{B}$ (Appendix A Eqs(A.78)). In the calculations this function is computed exactly in the self consistent field theory, while in the random phase approximation it is expanded in $\Psi$. Both theories neglect fluctuations. The gyroid phase ha not been studied in the random phase approximation.

The gyroid structure is stable for $\chi N$ between 14 and 20, which is in agreement with experiment. Please note that for $\chi N$ larger than 20 the double diamond structure is stable (see Fig.3). There is no direct transition between the disordered and gyroid structures. Instead at $f=0.452$ and $\chi N=11.14$ there is a triple point, where the lamellar, hexagonal and gyroid phase coexist. Below $\chi N=11.4$ the hexagonal phase transforms directly into the lamellar phase. In this approach the double diamond (obdd) structure is only metastable, but as we mentioned previously it is understable since the theory is applied to the weak segregation regime, while the obdd phase is expected at large $\chi N$ in the strong segregation regime.

The same method has been applied to the diblock copolymer system with 
conformational asymmetry, i.e. to the system where the length of the monomers $l$ is different for different types of monomers. The phase diagram in the case of $l_{A} / l_{B}=\sqrt{10}$ is show in Fig.16. By comparing the diagram in Fig.15 $\left(l_{A} / l_{B}=1\right)$ and Fig.16 we note that conformational asymmetry does not change the topology of the phase diagram ${ }^{25)}$.

\section{Other flexible block copolymer systems}

The variety of different block copolymer architectures is infinite. Therefore, here we shall chose only a few, to prove that the methods discussed so far can also be applied to block copolymers irrespective of the specific architecture. Here we discuss, in the random phase approximation, the triblock copolymers, diblock ring copolymers and random copolymers.

\section{A. Ring copolymers}

The AB diblock ring copolymer consists of two blocks joined in two points. The ring architeture can be easily included in the general scheme presented in section II. The same method as applied to polymer blends or linear diblock copolymers and described in Appendix A, B and C can be also applied to diblock ring copolymers. Here we consider the scattering intensity and the instability (same as in section IV A) in the system.

The scattering intensity $I(q)$ for ring copolymers, calculated in RPA, has exactly the same formal structure as given for linear diblock copolymers in section IV A (Eq.(4.3)). Only the matrix $S_{\alpha \beta}$ is different ${ }^{48)}$, i.e.

$$
\begin{aligned}
S_{11} & =\frac{N}{\rho_{0}} \int_{0}^{f} d s \int_{0}^{f} d s^{\prime} \mathrm{e}^{\left(-x\left|s-s^{\prime}\right|\right)\left(1-\left|s-s^{\prime}\right|\right)}, \\
S_{22} & =\frac{N}{\rho_{0}} \int_{f}^{1} d s \int_{f}^{1} d s^{\prime} \mathrm{e}^{\left(-x\left|s-s^{\prime}\right|\right)\left(1-\left|s-s^{\prime}\right|\right),} \\
S_{12} & =\frac{N}{\rho_{0}} \int_{0}^{f} d s \int_{f}^{1} d s^{\prime} \mathrm{e}^{\left(-x\left|s-s^{\prime}\right|\right)\left(1-\left|s-s^{\prime}\right|\right),}
\end{aligned}
$$


$x$ is defined after $\mathrm{Eq}(4.7)$. The same formulas are obtained for the linear diblock copolymers but without the term $1-\left|s-s^{\prime}\right|$ in the integrand. Due to this term the integrals cannot be calculated analytically for ring copolymers.

Let us first compare the scattering intensity of the linear and ring diblock copolymers in the case of $\chi=0$ (high temperature) and $f=1 / 2$ (symmetric case). In Fig. 17 the scattering intensities are shown. The peak in the ring copolymer is at larger q vector (which could be expected) and is less intense than the peak for the liner diblocks. In Fig.18 the instability line is shown (at which the scattering intensity diverges). As we see the rings require a larger amount of repulsion between $\mathrm{A}$ and $\mathrm{B}$ monomers to segregate. The latter result is also understandable since the block in the ring are more constrained than in the diblock. To see how severe are these constraints it is instructive to compare the instability in the ring copolymer to that in the $(A B)_{n}$ copolymer $\left.{ }^{80}\right)$ where there are $\mathrm{n} \mathrm{AB}$ diblocks joined together in the linear chain. The size of the $\mathrm{AB}$ diblock is $\mathrm{N}$ and each blocks has the size $\mathrm{N} / 2$ (symmetric case). It appears that in the limit of $n \rightarrow \infty$ the critical Flory Huggins parameter approaches a limiting value $\chi N \rightarrow 15$, whereas for the symmetric diblock rings (see Fig.18) of size $\mathrm{N}$ the instability occurs at $\chi N=17.8$, thus the transition occurs at even lower temperature. In the diblocks the same instability occurs at $\chi N=10.5$, thus the transition temperature to the lamellar phase is approximately $40 \%$ lower in the ring system than in the linear diblock copolymer system.

The result for the microphase separation obtained in the random phase approximation has been confirmed in the computer simulations ${ }^{47)}$. This result is in fact surprising, since in the random phase approximation it is assumed that chains adopt the Gaussian configurations, whereas in the ring system the constraints are such that the statistics is not Gaussian even in the limit of $N \rightarrow \infty$. The scaling exponent for the radius of gyration is $\nu=0.45\left(R_{g} \sim N^{\nu}\right)$ as has been found in 
computer simulations ${ }^{47,81,82}$ and theory ${ }^{466)}$. The comparison of the radius of gyration for the linear diblock chain and ring diblock chain is shown in Fig.19. Here $T_{c}$ is the transiton temperature to the lamellar phase and roughly concides with the instability temperature shown in Fig.18.

We have learned that the linear diblock copolymer system stretch as the temperature is lowered ${ }^{20,21)}$. The same applies to ring diblock copolymers ${ }^{47)}$. As the temperature is lowered the strong increase (20-25\%) in the distance between the center of masses of two blocks is observed (Fig.20). At the same time the blocks themselves shrink (Fig.21) similarly as in the case of linear diblock copolymer system discussed in section IV. The effect of the stretching is more pronounced in the rings (20-25\% above the radius of gyration at the infinite temperature) than in the linear diblocks (15\% above the high temperature radius of gyration).

\section{B. Triblock copolymers}

The ABA triblock copolymer system has been studied by several authors ${ }^{83-86)}$ The phase diagram for this system is similar to the one observed in diblock copolymers $^{84,85)}$ (Fig.22). This observation has been supported by experiments ${ }^{83,87-89)}$. This is not surprising since cutting the triblock in the middle produces two diblock coplymers and the entropy gained from doing this should not be very large, so the free energy of triblocks in a particular phase should be roughy the same as that of twice as many diblocks of half the length in their analogous phase.

The scattering intensity for the triblock copolymer system has the same general form as given by $\operatorname{Eq}(4.3)$, only the matrix $S_{\alpha \beta}$ is different ${ }^{83)}$ ( $g_{1}$ is defined in $\operatorname{Eq}(4.7))$ i.e.

$$
\begin{aligned}
S_{11} & =\frac{N}{\rho_{0}}\left(g_{1}\left(f_{1}, N\right)+g_{1}\left(f_{2}, N\right)+g_{1}\left(f_{3}, N\right)\right. \\
& \left.+g_{1}(1, N)-g_{1}\left(1-f_{3}, N\right)-g_{1}\left(1-f_{1}, N\right)\right)
\end{aligned},
$$




$$
\begin{gathered}
S_{22}=\frac{N}{\rho_{0}} g_{1}\left(f_{2}, N\right), \\
S_{12}=\frac{N}{2 \rho_{0}}\left(g_{1}\left(1-f_{1}, N\right)+g_{1}\left(1-f_{3}, N\right)+g_{1}(1, N)\right. \\
\left.-g_{1}\left(f_{3}, N\right)-g_{1}\left(f_{1}, N\right)-2 g_{1}\left(f_{2}, N\right)\right)
\end{gathered}
$$

Here $f_{1}, f_{2}, f_{3}$ are the monomer fractions in each block, 1,3 are A blocks.In the case of $f_{3}=0$ (or $f_{1}=0$ ) the formula reduces to the diblock copolymer formula given in $\operatorname{Eqs}(4.4-4.6)$.

We can also expect that as we lower the temperature the chains stretch, but the individual blocks shrink.

There is one notable difference between the system of ABA triblocks and AB diblocks. In the former case there are bridge and loop configurations (Fig.4), depending on the location, in lamellas, of the different A blocks belonging to the same triblock. The presences of bridges should affect the mechanical properties of the triblock system $^{86)}$ or in general the multi-block copolymer system ${ }^{44)}$. It has been found that about 40-50\% of copolymers form bridges in the triblock copolymer lamellar phase.

\section{Random copolymers}

In the $\mathrm{AB}$ random copolymer system ${ }^{40)}$ the architecture of the chain is specified not only by the fraction of A monomers in the chain, $f$, but also by the conditional probabilities that the $\mathrm{A}$ monomer in the chain is followed by the $\mathrm{A}, p_{A A}$, or $\mathrm{B}$ monomer, $p_{A B}$ and similarly for $\mathrm{B}$ monomers. One naturaly has $p_{A A}+p_{A B}=1$ and same for B. The function $f$ and $\lambda=p_{A A}+p_{B B}-1$ are sufficient to describe the disorder quenched into an AB random copolymer. Now apart from the densities already introduced in section II we have to include the occupation number $\theta_{i}$ at the i-th point along the chains. As in all the systems with quenched disorder one has to calculate all the relevant thermodynamic quantities for fixed distribution of $\theta$ and 
then average them with respect to $\theta$, which describes the disorder. The practical way to do it is by the replica method $40-43,90)$.

Let us study the macrophase separation in the melt, which is similar to the separation in A, B homopolymer blend. The condition for the instability in the melt in the random phase approximation is:

$$
4 f(1-f) \chi_{c}^{m f}=\frac{2 N(1-\lambda)^{2}}{N(-\lambda)^{2}+2 \lambda\left(N(1-\lambda)+\lambda^{N}-1\right)} .
$$

For $\lambda \rightarrow 1$ this condition reduces to the same equation as we would get for the mixture of A homopolymers and B homopolymer each of length $N l$ i.e.

$$
4 f(1-f) \chi_{c}^{m f}=\frac{2}{N}
$$

In the limit of $\lambda \rightarrow 0$ we would have a system where the copolymers have the very short sequences of the A and B monomers. Thus the condition (5.7) reduces to

$$
4 f(1-f) \chi_{c}^{m f}=2
$$

as for the system of disconnected monomers. As we see in this limit the interesting scale of the phenomena is related to the short sequence of the A or B monomers and not to the size of the polymer molecule. Consequently our description is no longer valid; in particular the structure and phase transitions in the system depend on the details of the intermolecular potential, which in the present treatment have been neglected.

\section{Mixtures of homopolymers and diblock copolymers}

So far we have considered the polymer melts or blends which were described by a single order parameter. In order to illustrate the application of the method presented here to more complicated polymer blends we consider a ternary mixture of A, B homopolymers and AB diblock copolymers ${ }^{34-37)}$. This system is in some respects 
similar to the ternary mixture of oil water and amphiphiles ${ }^{38,91)}$. The copolymer simply acts as a surfactant accumulating at the interface of A-homopolymer rich phase and B-homopolymer rich phase, reducing in this way the number of unfavorable contacts between $\mathrm{A}$ and $\mathrm{B}$ monomers ${ }^{36,92)}$. This analogy will be exploited here.

\section{A. Structure factor matrix in the RPA}

To simplify the calculations we assume that the length of all the polymers is $\mathrm{N}$ and the diblocks are symmetric. It is convenient to introduce a set of three order parameters. The first order parameter, $\eta$, measures the deviation from its average value of the concentration difference of $\mathrm{A}$ and $\mathrm{B}$ homopolymer. The second $\varepsilon$, measures the local deviation from its average value of the copolymer concentration. The third, $\Psi$, measures the local deviation from its average value of the total fraction of A-monomers. All order parameters vanish in a spatially uniform, one phase region.

Now the scattering intensity

$$
I(q)=\left(\Gamma_{2}^{(0)}(q)\right)^{-1}
$$

becomes a matrix and so does the vertex function $\Gamma_{2}^{(0)}$. For the matrix

$$
\Gamma_{2}^{(0)}=\left|\begin{array}{ccc}
\Gamma_{\eta \eta} & \Gamma_{\eta \varepsilon} & \Gamma_{\eta \Psi} \\
\Gamma_{\eta \varepsilon} & \Gamma_{\varepsilon \varepsilon} & 0 \\
\Gamma_{\eta \Psi} & 0 & \Gamma_{\Psi \Psi}
\end{array}\right|
$$

calculated in the random phase approximation (Appendix A,B) we obtain

$$
\begin{gathered}
\Gamma_{\eta \eta}=\frac{1}{4}\left(\frac{1}{S_{A}}+\frac{1}{S_{B}}+\frac{2}{S_{A A}-S_{A B}}\right), \\
\Gamma_{\eta \varepsilon}=\frac{1}{4}\left(\frac{1}{S_{A}}-\frac{1}{S_{B}}\right),
\end{gathered}
$$




$$
\begin{gathered}
\Gamma_{\eta \Psi}=-\left(\frac{1}{S_{A A}-S_{A B}}\right) \\
\Gamma_{\varepsilon \varepsilon}=\frac{1}{4}\left(\frac{1}{S_{A}}+\frac{1}{S_{B}}+\frac{2}{S_{A A}+S_{A B}}\right), \\
\Gamma_{\Psi \Psi}=\frac{2}{S_{A A}-S_{A B}}-2 \chi
\end{gathered}
$$

where

$$
\begin{gathered}
S_{A}=\frac{(1-\phi)}{2 \rho_{0}} N g_{1}(1, x), \\
S_{B}=\frac{(1-\phi)}{2 \rho_{0}} N g_{1}(1, x), \\
S_{A A}=\frac{\phi}{\rho_{0}} N g_{1}(1 / 2, x), \\
S_{A B}=\frac{\phi}{2 \rho_{0}} N\left(g_{1}(1, x)-2 g_{1}(1 / 2, x)\right),
\end{gathered}
$$

and $\phi$ is the average concentration of copolymer. For simplicity we have assumed that the concentrations of homopolymers A and homopolymers B are equal. Function $g_{1}$ is given by $\operatorname{Eq}(4.7)$.

The two most useful scattering intensities, are obtained by taking the inverse of the vertex function matrix $\operatorname{Eq}(6.1)$, and are given below:

$$
I_{\Psi \Psi}=\frac{\left(S_{A}+S_{A A}-S_{A B}\right)}{2\left[1-\rho_{0} \chi\left(S_{A}+S_{A A}-S_{A B}\right)\right]},
$$

in agreement with Broseta and Fredrickson ${ }^{34)}$ and

$$
I_{\eta \eta}=\frac{2 S_{A}\left[1-\chi\left(S_{A A}-S_{A B}\right)\right]}{\left[1-\rho_{0} \chi\left(S_{A}+S_{A A}-S_{A B}\right)\right]},
$$

in agreement with Leibler ${ }^{92)}$.

For completeness, we compute also the copolymer copolymer structure function at equal homopolymer concentrations,

$$
\begin{aligned}
I_{\varepsilon \varepsilon} & =2\left(\frac{1}{S_{A}}+\frac{1}{S_{A A}+S_{A B}}\right)^{-1} \\
& =N g_{1}(1, x) \phi(1-\phi) / \rho_{0} .
\end{aligned}
$$


This result, in agreement with Leibler ${ }^{92)}$, is independent of temperature, and always has a peak only at zero wavevector only. In this respect it is similar to calculated amphiphile-amphiphile structure functions in systems of oil, water and surfactant $^{38,94)}$.

\section{B. Stability limits, disorder, Lifshitz and equimaxima lines}

Much of the phase diagram can be obtained from either of the above structure functions. The limit of stability of the disordered phase occurs at the highest temperature at which a divergence in the above structure functions appears. From Eqs. (6.12) or (6.13), we see that this happens at the smallest value of $\chi$. Here we treat $\chi$ as a function of $x$ so that we can minimize it with respect to $x$. From the denominator of the structure functions we find

$$
\frac{1}{\rho_{0} \chi(x) N}=\frac{1}{N}\left(S_{A}(x)+S_{A A}(x)-S_{A B}(x)\right),
$$

or, using the explicit formulas,

$$
\frac{1}{\chi(x) N}=\frac{e^{-x}(1-2 \phi)+4 \phi e^{-x / 2}+x-1-2 \phi}{x^{2}},
$$

where again, $x=q^{2} R^{2} \equiv q^{2} N^{2} l^{2} / 6$. Minimizing $\chi(x)$, we find that for $\phi \leq 2 / 3$, the minimum occurs at $x=0$, and that its value is

$$
\chi N=\frac{2}{1-\phi}, \quad \phi \leq 2 / 3
$$

Along this line, the disordered phase makes a continuous transition to uniform $(q=0)$ phases. This is just the macroscopic phase separation into A-rich and Brich phases. For a copolymer density between $2 / 3$ and 1 , the instability occurs at a non-zero value of $x$ and the continuous transition is to a phase characterized by the corresponding non-zero wavevector $(x)^{1 / 2} / R$; this is the microphase separation to 
a lamellar phase. The density of copolymer at which a transition occurs to a phase characterized by $x \neq 0$ is

$$
\phi(x)=\frac{-2+x+(x+2) e^{-x}}{2\left[2+(x+2) e^{-x}-(x+4) e^{-x / 2}\right]}, \quad 0<x \leq 3.785
$$

and the value of $\chi N$ at which it occurs is given by Eqs. (6.16) with $x$ in the range indicated in Eq. (6.18). The transition lines are shown in Fig. 23. There is a region of three phase coexistence between the A-rich, B-rich and lamellar phases which is shown schematically in the figure.

Much more information about the disordered phase can be extracted from the structure functions, of course. To obtain some information on how the copolymer tends to order the homopolymer and also to order itself, it is useful to introduce the disorder line and the Lifshitz lines ${ }^{35,38,39)}$. The disorder line is the locus of points in the phase diagram at which a general correlation function, $G(\mathbf{r})$, no longer decays monotonically with distance at large $r$, but acquires a component which, at large distances, behaves like an exponentially decaying oscillatory function;

$$
G(\mathbf{r}) \rightarrow \frac{\exp (-r / \xi)}{r} \sin (2 \pi r / \lambda+\delta)
$$

This oscillatory behavior reflects the tendency of the copolymer to order the A and B monomers in space. Thus, on the copolymer-rich side of the disorder line, two lengths are needed to describe the disordered phase; the usual correlation length $\xi$, and a wavelength $\lambda$. As the amount of copolymer in the system is increased, one expects the wavelength to decrease as order can be enforced over shorter distances; conversely, as the copolymer density decreases, $\lambda$ increases and diverges at the disorder line. We locate the disorder line as follows. The Fourier transform of a general correlation function can be written as a linear combination of the $I_{i j}(q)$, and the poles of these functions determine the behavior of the correlation function. 
If these poles are located at a value of $q$ which is solely imaginary, the correlation function decays monotonically with distance. Thus the disorder line is the locus at which these poles first acquire a non-zero real part. As $I_{i j}=\left(\Gamma_{2}^{(0)}\right)_{i j}^{-1}$, the location of these poles is also the locus at which the determinant $\left|\Gamma_{2}^{(0)}(q)\right|$ vanishes for imaginary $q$. Because this determinant is common to all $I_{i j}$, all correlation functions have the same disorder line in general. From the structure functions $I_{\Psi \Psi}$ or $I_{\eta \eta}$ we see that the disorder line is found by locating the poles of either correlation function, just as the phase boundary was located. However to locate the phase boundary, we sought the poles occurring for real $q$ (corresponding to $x \geq 0$ ), whereas to locate the disorder line we seek the poles occurring for $q$ just as it becomes purely imaginary (corresponding to $x \leq 0$ ). We do this by setting $x=-y+i \delta, y \geq 0$ in Eq. (6.16), and equating real and imaginary parts. Then taking the limit of $\delta$ to zero, we obtain two equations which can be solved for $\phi(y)$ and $\chi(y) N$ on the disorder line. They are

$$
\phi(y)=\frac{\left(e^{y}(2-y)-y-2\right)}{2\left[e^{y}(2-y)-e^{y / 2}(4-y)+2\right]},
$$

and

$$
\frac{1}{\chi(y) N}=\frac{e^{3 y / 2}-e^{y}(y+1)+e^{y / 2}(y-1)+1}{y\left[e^{y / 2}(4-y)-2-e^{y}(2-y)\right]},
$$

where $y$ ranges over all positive real numbers. Two limits readily emerge from these equations. They are that $\phi$ approaches $2 / 3$ as $\chi N$ approaches 6 , and that $\phi$ approaches $1 / 2$ as $\chi N$ vanishes. The disorder line is shown in the phase diagram of Fig. 24 as the line LD.

The disorder line shows where oscillatory behavior first appears in the correlation functions of the disordered system. The locus at which this behavior first dominates a particular correlation function is given by one of two kinds of line. The first is the Lifshitz line; at this line, the peak in the associated structure function 
moves continuously from zero to a non-zero wavevector. Its location is determined by

$$
\left.\frac{\partial^{2} I_{i j}(\mathbf{q})}{\partial q^{2}}\right|_{q=0}=0
$$

In general, each structure function has its own, distinct, Lifshitz line, and the difference in these lines provides information on the different effects the copolymer has on the various components of the system. The Lifshitz line associated with the structure function of all A monomers, is readily found to be given by $\phi=2 / 3$, independent of temperature. This line is shown as LF in Fig. 24. The fact that the disorder line and the Lifshitz line of $I_{\Psi \Psi}$ are close indicates that once the tendency of the copolymers to order the A monomers appears in the correlation functions, relatively few additional copolymers are needed to make this tendency dominant in the total A, total A correlation function. It is not clear, however, whether the copolymer affects the A monomers in the copolymers or in the homopolymers more strongly, or whether it affects them equally. This can be determined by examining $I_{\eta \eta}$, which depends only on the homopolymer concentration, and finding the locus at which this function is dominated by components of non-zero wavenumber. In the vicinity of the point $\chi N=6, \phi=2 / 3$, this dominance is seen as the peak at zero wavevector moves to nonzero q, a movement which occurs at the Lifshitz line of this structure function;

$$
\frac{1}{\chi N}=\left[\frac{(1-\phi) \phi}{8}\right]^{1 / 2}
$$

However, another mechanism takes over beyond $\phi \approx 0.79$. One finds instead that a second peak at non-zero q develops in $I_{\eta \eta}$, and becomes larger than the peak at $q=0$. This occurs before the Lifshitz line condition, Eq. (6.22), is satisfied. This new line, which we refer to as an equimaxima line, is defined as the locus of points at which the two peaks, one at zero, the other at non-zero wavenumber, are of equal 
height. At this line, the wavevector characterizing which is the largest peak in the structure function moves discontinuously from zero. The line is determined by the simultaneous solution of

$$
\left.\frac{\partial I_{\eta \eta}(x, \phi, \chi)}{\partial x}\right|_{x^{*}}=0
$$

and

$$
I_{\eta \eta}\left(x^{*}, \phi, \chi\right)=I_{\eta \eta}(0, \phi, \chi)
$$

The equimaxima line (EG on Fig. 24) and Lifshitz line (LE) join smoothly as $x^{*} \rightarrow 0$ which occurs at $\phi=121 / 153 \approx 0.79$ (point E). We clearly see from Fig.24 that even though oscillatory components enter all correlation functions at the disorder line and dominate the correlation function of all A monomers with only a rather small additional increase of copolymer concentration, they do not dominate the correlation function of those monomers only in the homopolymers until a large increase of copolymer concentration, so large that the system is about to become unstable to the lamellar phase. We conclude, therefore, that the copolymer is very efficient in self organization, but very inefficient in the organization of homopolymers.

\section{The Landau-Ginzburg free energy and the Lifshitz point}

To simplify the calculations we consider the case of equal homopolymer concentrations and integarte out the concentration of copolymers $\varepsilon$. Therefore we are left with one single order parameter $\eta$. We find a Landau free energy in the following form:

$$
\Omega[\eta]=\frac{1}{2} k_{B} T \rho_{0} \int d \mathbf{r}\left(h_{0}\left|\nabla^{2} \eta(\mathbf{r})\right|^{2}+g_{0}|\nabla \eta(\mathbf{r})|^{2}+f(\eta)\right)
$$

where the bulk free energy is:

$$
f(\eta)=a \eta^{2}+b \eta^{4}+c \eta^{6}
$$

Here $g_{0}$ and $h_{0}$ are constants indepent on $\eta$, but in general they do depend ${ }^{36)}$ on $\eta\left(h(\eta=0)=h_{0}\right.$ and $\left.g(\eta=0)=g_{0}\right)$. The parameters $a, b, c$ and $h_{0}, g_{0}$ are given 
in the Appendix G. An ordinary critical point occurs when $a=0$, while the other parameters are positive, and an ordinary tricritical point occurs when $a=0$ and $b=0$, with the other parameters positive. Because $g_{0}$ is positive, the ordered states which occur just below these transitions are spatially uniform, characterized by a vanishing wavevector $q$. At a Lifshitz point, the stability of these uniform states is lost because $g_{0}=0$ there, so that these points mark the last appearance of uniform ordered states and the first appearance of non-uniform ordered states (e.g. a lamellar one) characterized by a non-zero wavevector. At a Lifshitz critical point, $a=g_{0}=0$; similarly at a Lifshitz tricritical point, $a=b=g_{0}=0$, with all other parameters positive. One sees immediately how unusual this point is expected to be as three coefficients must manage to vanish simultaneously. As you can see from the results given in Appendix G the point L on Fig.24 is the Lifshitz tricritical point.

We first determine the behavior of the uniform solution, $\eta_{0}$, as the Lifshitz tricritical point located at $T_{c}$ is approached $\left(g_{0}=b=0, a \sim t \equiv\left(T-T_{c}\right) / T_{c}\right)$ by variation of the free energy of $\operatorname{Eq}(1.1)$ and find $\eta_{0} \sim t^{\beta}$ with $\beta=1 / 4$. Inserting this into Eq. (6.26), we find $F \sim t^{2-\alpha}$ with $\alpha=1 / 2$. These exponents also characterize the ordinary tricritical point in mean field theory. Thus the Lifshitz nature of the tricritical point does not affect these exponents within mean field theory. To see the difference between the Lifshitz and the ordinary tricritical point, we look at the behavior of deviations of the order parameter from uniformity. We find that $\Gamma_{2}^{(0)}(\mathbf{q} \rightarrow 0) \sim t^{\gamma}$ with $\gamma=1$, again independent of the Lifshitz phenomena. However, if we rescale the wavevector $\mathbf{q}$ by the correlation length $\xi$, we find that $\xi \sim t^{-\nu}$ with $\nu=1 / 4$ in contrast to ordinary critical behavior in which, with $g_{0}$ nonzero, $\nu$ would be $1 / 2$. The Lifshitz behavior also is manifest in the value of the upper critical dimension, $d^{*}$, the smallest dimension at which mean field behavior is correct. For ordinary tricritical behavior, $d^{*}=3$, so that fluctuations 
are negligible. (They contribute logarithmic corrections to mean field results.) For Lifshitz tricritical behavior, this is not so as $d^{*}$ is greater than 3 . To determine this dimension, we employ a simple scaling argument applicable whenever fluctuations dominate the critical behavior. We rescale the order parameter $\eta$ by its bulk, uniform, value $\eta_{0}$ and the position vector $\mathbf{r}$ by the correlation length $\xi$ in Eq.(6.26). Again, $g_{0}$ and $b$ are set to zero so that the Lifshitz tricritical point is approached. Requiring that the coefficient of the Laplacian squared term be independent of the reduced temperature $t$, we obtain $\eta_{0}=\xi^{(4-d) / 2} \sim t^{(d-4) \nu / 2}$, where $d$ is the dimension of space. However $\eta_{0} \sim t^{\beta}$ by the definition of the exponent $\beta$. Requiring the equality of these two exponents when the mean field values of $\beta=1 / 4$ and $\nu=1 / 4$ are inserted determines $d^{*}=6$. Below this dimension, fluctuation effects are important, and the exponents differ from their classical values. These exponents are as yet unknown theoretically as well as experimentally, so that a measurement of any of them would be of considerable interest. One reason why they have not been calculated is that the usual epsilon expansion for this system fails at dimension $\mathrm{d}=4$. This can be seen by considering a term of the form $\eta^{p}$ in the integrand of Eq (6.26). Again rescaling $\eta$ by $\eta_{0}$ and lengths by $\xi$ one finds the coefficient of such a term to be $\xi^{d} \xi^{p(4-d) / 2}$. At $\mathrm{d}=4$, every term of the form $\eta^{p}$ has the same coefficient $\xi^{d}$. This means that one should use the whole series in $\eta$ to perform reliable calculations, which would result in an infinite number of renormalization group flow equations for the renormalized constants.

Although the predictions of mean field theory fail at the Lifshitz tricritical point and within a region close to it, the predictions are valid outside this region, so that it is important to know its extent. This can be estimated with the help of the Ginzburg criterion. We follow de Gennes ${ }^{3)}$ and Joanny ${ }^{5}$ and assume that fluctuations are unimportant if the fluctuations of the order parameter in a correlation volume is 
much smaller than characteristic changes in the square of the order parameter itself, i.e. if

$$
<(\delta \eta)^{2}>/ \eta_{0}^{2} \propto 1 / \Gamma_{2}^{(0)}(q \rightarrow 0) \xi^{d} \eta_{0}^{2} \ll 1
$$

Therefore, mean field theory is valid for temperatures $t_{*}$ such that

$$
\frac{1}{\Gamma_{2}^{(0)}(q \rightarrow 0) \xi^{d} \eta_{0}^{2}} \sim \frac{t_{*}^{d \nu-\gamma-2 \beta}}{N^{d / 2-1}} \ll 1,
$$

where $\beta, \nu$ and $\gamma$ are the mean field exponents. The $\mathrm{N}$ dependence follow from the fact that in polymers $\xi \sim N^{1 / 2}$ and $\Gamma(0) \sim N$, where $\mathrm{N}$ is the number of monomers in the polymer. Here we consider a system where all polymers have the same number of monomers. For $d=3$, fluctuations can be ignored near the Lifshitz tricritical point provided that

$$
t_{*} \gg 1 / N^{2 / 3} \text {. }
$$

Similarly, $t_{*} \gg 1 / N$ for an ordinary critical point (see section III) and $t_{*} \gg 1 / N^{2 / 5}$ for a Lifshitz critical point. In the limit $\mathrm{N}$ to infinity, mean field theory is valid in the entire critical region near the Lifshitz tricritical point. The influence of the Lifshitz on the surface tension of the coexisting A-rich and B-rich phases (see Fig.23) is discussed in Appendix H. The elastic properties of the interface between the A-rich and B-rich phases can be find in Ref.[36].

\section{Rigid-flexible diblock copolymer system}

So far we have considered only the flexible systems. From Section II we have learned that also the rigid polymers can be described using this method. In this section we discuss the rigid-flexible diblock copolymer system. The order parameter densities have been already discussed in section II.D. They shall be used in the following subsections. 


\section{A. The scattering intensity}

The scattering matrix has the following form in the case of rigid flexible diblock copolymers $^{50)}$ :

$$
I_{i j}(\mathbf{q})=\left(\Gamma_{2}^{(i n t)}(\mathbf{q})+\Gamma_{2}^{(0)}(\mathbf{q})\right)_{i j}^{-1}
$$

Here

$$
\begin{aligned}
& \left(\Gamma_{2}^{(0)}(\mathbf{q})\right)^{-1}=I^{(0)}(\mathbf{q})= \\
& \quad \frac{1}{V}\left|\begin{array}{cccc}
<\hat{\phi}_{A}^{(n)} \hat{\phi}_{A}^{(n)}>_{0} & <\hat{\phi}_{A}^{(n)} \hat{\phi}_{B}^{(n)}>_{0} & <\hat{\phi}_{A}^{(n)} \hat{Q}_{\alpha \beta}^{(B)}>_{0} & <\hat{\phi}_{A}^{(n)} \hat{Q}_{\alpha \beta}^{(A)}>_{0} \\
<\hat{\phi}_{B}^{(n)} \hat{\phi}_{A}^{(n)}>_{0} & <\hat{\phi}_{B}^{(n)} \hat{\phi}_{B}^{(n)}>_{0} & <\hat{\phi}_{B}^{(n)} \hat{Q}_{\alpha \beta}^{(B)}>_{0} & <\hat{\phi}_{B}^{(n)} \hat{Q}_{\alpha \beta}^{(A)}>_{0} \\
<\hat{Q}_{\alpha \beta}^{(B)} \hat{\phi}_{A}^{(n)}>_{0} & <\hat{Q}_{\alpha \beta}^{(B)} \hat{\phi}_{B}^{(n)}>_{0} & <\hat{Q}_{\gamma \delta}^{(B)} \hat{Q}_{\alpha \beta}^{(B)}>_{0} & <\hat{Q}_{\gamma \delta}^{(B)} \hat{Q}_{\alpha \beta}^{(A)}>_{0} \\
<\hat{Q}_{\alpha \beta}^{(A)} \hat{\phi}_{A}^{(n)}>_{0} & <\hat{Q}_{\alpha \beta}^{(A)} \hat{\phi}_{B}^{(n)}>_{0} & <\hat{Q}_{\alpha \beta}^{(A)} \hat{Q}_{\gamma \delta}^{(B)}>_{0} & <\hat{Q}_{\gamma \delta}^{(A)} \hat{Q}_{\alpha \beta}^{(A)}>_{0}
\end{array}\right|
\end{aligned}
$$

is the ideal part of the inverse vertex function and is solely determined by the conformations of a single copolymer molecule. Matrix (7.2) is a symmetric twelve by twelve matrix. By the direct calculations of the ideal averages, one may convince oneself that the averages involving the nematic tensor for the flexible chain, $Q_{\alpha \beta}^{(A)}(\mathbf{r})$, are $\mathrm{N}_{0}\left(\mathrm{~N}_{0}=\mathrm{N}_{B}\right.$ or $\left.\mathrm{N}_{A}\right)$ times smaller than the other averages. This is understandable since flexible chains consist of freely joined bonds, thus ordering one bond does not affect the ordering of the other bonds. In contrast, in the rigid rod, ordering one bond automatically orders the whole rigid B-part of the diblock copolymer. This allow us to neglect $Q_{\alpha \beta}^{(A)}$ in our calculations and in the matrix(7.2). Thus $I^{(0)}$ is reduced to a seven by seven matrix. Futhermore, by chosing the coordinate frame in which the $z$ axis is along the $\mathbf{q}$ vector, $I^{(0)}$ simplifies to the following block form:

$$
I^{(0)}=\left|\begin{array}{cc}
\Lambda & 0 \\
0 & \Delta
\end{array}\right| \text {. }
$$

Here

$$
\begin{aligned}
& \Lambda= \\
& \frac{1}{V}\left|\begin{array}{cccc}
<\hat{\phi}_{A}^{(n)} \hat{\phi}_{A}^{(n)}>_{0} & <\hat{\phi}_{A}^{(n)} \hat{\phi}_{B}^{(n)}>_{0} & <\hat{\phi}_{A}^{(n)} \hat{Q}_{x x}^{(B)}>_{0} & <\hat{\phi}_{A}^{(n)} \hat{Q}_{y y}^{(B)}>_{0} \\
<\hat{\phi}_{B}^{(n)} \hat{\phi}_{A}^{(n)}>_{0} & <\hat{\phi}_{B}^{(n)} \hat{\phi}_{B}^{(n)}>_{0} & <\hat{\phi}_{B}^{(n)} \hat{Q}_{x x}^{(B)}>_{0} & <\hat{\phi}_{B}^{(n)} \hat{Q}_{y y}^{(B)}>_{0} \\
<\hat{Q}_{x x}^{(B)} \hat{\phi}_{A}^{(n)}>_{0} & <\hat{Q}_{x x}^{(B)} \hat{\phi}_{B}^{(n)}>_{0} & <\hat{Q}_{x x}^{(B)} \hat{Q}_{x x}^{(B)}>_{0} & <\hat{Q}_{x x}^{(B)} \hat{Q}_{y y}^{(B)}>_{0} \\
<\hat{Q}_{y y}^{(B)} \hat{\phi}_{A}^{(n)}>_{0} & <\hat{Q}_{y y}^{(B)} \hat{\phi}_{B}^{(n)}>_{0} & <\hat{Q}_{y y}^{(B)} \hat{Q}_{x x}^{(B)}>_{0} & <\hat{Q}_{y y}^{(B)} \hat{Q}_{y y}^{(B)}>_{0}
\end{array}\right|,
\end{aligned}
$$


is a four by four matrix, whereas $\Delta$ is a diagonal three by three matrix,

$$
\Delta=\frac{1}{V}\left|\begin{array}{ccc}
<\hat{Q}_{x y}^{(B)} \hat{Q}_{x y}^{(B)}>_{0} & 0 & 0 \\
0 & <\hat{Q}_{x z}^{(B)} \hat{Q}_{x z}^{(B)}>_{0} & 0 \\
0 & 0 & <\hat{Q}_{y z}^{(B)} \hat{Q}_{y z}^{(B)}>_{0}
\end{array}\right|
$$

Because the off diagonal elements of $\hat{Q}_{\alpha \beta}^{(B)}$ appears only in $\Delta$, they can be integrated out immediately in the integrals (see Appendix A,B).

After the inversion of $I^{(0)}$, we will make use of the incompressibility condition which, in Fourier space, reads

$$
\phi_{A}^{(n)}(\mathbf{q})+\phi_{B}^{(n)}(\mathbf{q})=0
$$

for $\mathbf{q} \neq 0$. After these algebraic manipulations, we end up with the following form of the ideal and interaction vertex function:

$$
\Gamma_{2}^{(0)}=\left|\begin{array}{ccc}
\Lambda_{11}^{-1}-2 \Lambda_{12}^{-1}+\Lambda_{22}^{-1} & \Lambda_{23}^{-1}-\Lambda_{13}^{-1} & \Lambda_{24}^{-1}-\Lambda_{14}^{-1} \\
\Lambda_{23}^{-1}-\Lambda_{13}^{-1} & \Lambda_{33}^{-1} & \Lambda_{34}^{-1} \\
\Lambda_{24}^{-1}-\Lambda_{14}^{-1} & \Lambda_{34}^{-1} & \Lambda_{44}^{-1}
\end{array}\right|
$$

and

$$
\Gamma^{(i n t)}=\rho_{0}\left|\begin{array}{ccc}
-2 \chi & 0 & 0 \\
0 & -\frac{4}{3} v_{B B} & -\frac{2}{3} v_{B B} \\
0 & -\frac{2}{3} v_{B B} & -\frac{4}{3} v_{B B}
\end{array}\right| .
$$

Here $\Lambda_{i j}^{-1}$ is the ij element of the inverse of $\Lambda$. This matrix is also symmetric.

To summarize this section, we have been able to reduce the set of twelve order parameters to the relevant set of only three order parameters, namely the two previously mentioned components of the nematic tensor i.e. $Q_{x x}^{(B)}(\mathbf{q}), Q_{y y}^{(B)}(\mathbf{q})$ and $\Psi(\mathbf{q})$.

\section{B. Stability limits of the isotropic phase}

At high temperatures the scattering matrix given by $\operatorname{Eqs}(7.1-8)$ is positive definite thus the system is stable with respect to compositional, and nematic perturbations. However, as we lower the temperature the scattering matrix ceases to 
be positive definite and the isotropic phase becomes unstable. We find the stability conditions by equating the determinant of the scattering matrix or its principal minors to zero. The results of this stability analysis are presented in Fig.25 as a plot of $\chi N$ versus the fraction of A monomers in the copolymer $f=N_{A} / N$. If all phase transitions were continuous, these figures would be phase diagrams. First we discuss the case in which there is no explicit interaction tending to orient the rigid rods, $v_{B B}=0$. This is shown in Fig. 25a. One can easily see that the nematic order parameters can be integrated out in this case, and the stability condition is

$$
\Lambda_{11}+\Lambda_{22}+2 \Lambda_{12}-2\left(\Lambda_{11} \Lambda_{22}-\Lambda_{12}^{2}\right) \rho_{0} \chi=0, .
$$

The phase diagram encompasses in this case the disordered isotropic phase and some spatially ordered phases; for convenience we will call all of them lamellar phases. The stability analysis within our random phase approximation does not give us the symmetry of these phases. The stability condition against lamellar perturbations for the rigid-flexible system of the diblock copolymers $(\operatorname{Eq}(7.9))$ has the same formal structure as that obtained in the Random Phase Approximation by Leibler ${ }^{22)}$ for the flexible-flexible diblock copolymer system. The difference between this condition and the one obtained by Leibler is in the ideal averages which, in our case, reflect the rigid-flexible structure of the polymer (see Appendix I). Let us discuss the differences in the stability limits for these two systems. First of all we note that in the rigid-flexible copolymer system the stability temperature is higher (or the interaction Flory-Huggins $\chi$ parameter lower) in comparison to the systems of perfectly flexible system compare Fig.25a and Fig.12. For example below $\chi N=9$, our system is stable for all f; while the flexible-flexible diblock copolymer system is only stable below $\chi N=10.5$. This reflects the reduction in entropy due to the rigidity of part of the polymer. In addition, the mimimum of the curve shown in 
Fig. 25 a occurs at $\mathrm{f}=0.45$ whereas in the flexible copolymers it occurs at $\mathrm{f}=0.5$. The results of this calculations find support in the computer simulation ${ }^{94)}$.

For a system with nonzero anisotropic interaction parameter, $v_{B B}$, a nematic phase appears in addition to the lamellar and isotropic phases (Figs.25 bc)). The dashed line shown in Figs.25bc denotes the stability limits against nematic perturbations. It satisfies the following equation

$$
\frac{5}{2 N(1-f)^{2}}-\frac{v_{B B}}{2}=0
$$

Furthermore we observe that the stability limit against lamellar perturbations goes towards higher temperatures as the anisotropic interaction parameter increases, although the change is rather small, a few percent at most. The black dot in the figures denotes the tricritical point at which the isotropic phase is unstable against both nematic and lamellar perturbations at the same temperature. As the transitions in the system are actually first order, this tricritical point is preempted by a triple point at which all three phases coexist. The calculated tricritical point is an estimate of the location of the actual triple point.

\section{The correlation functions}

We have calculated the following correlation functions for $\mathbf{q}=\left(0,0, q_{z}\right)$ :

$$
\begin{gathered}
\tilde{G}_{\Psi \Psi}\left(q_{z}\right)=\frac{1}{V}<\hat{\Psi}\left(q_{z}\right) \hat{\Psi}\left(-q_{z}\right)>; \\
\tilde{G}_{\Psi Q}\left(q_{z}\right)=\frac{1}{V}<\hat{\Psi}\left(q_{z}\right) \hat{Q}_{z z}^{(B)}\left(-q_{z}\right)>; \\
\tilde{G}_{Q Q}\left(q_{z}\right)=\frac{1}{V}<\hat{Q}_{z z}^{(B)}\left(q_{z}\right) \hat{Q}_{z z}^{(B)}\left(-q_{z}\right)>;
\end{gathered}
$$

and the following Fourier transforms of these correlation functions:

$$
\begin{aligned}
G_{\Psi \Psi}(z)= & \int d x \int d y<\hat{\Psi}(\mathbf{r}) \hat{\Psi}(0)> \\
& =\frac{1}{2 \pi} \int d q_{z} \tilde{G}_{\Psi \Psi}\left(q_{z}\right) \exp \left(i q_{z} z\right)
\end{aligned}
$$




$$
\begin{aligned}
G_{\Psi Q}(z)= & \int d x \int d y<\hat{\Psi}(\mathbf{r}) \hat{Q}_{z z}^{(B)}(0)> \\
& =\frac{1}{2 \pi} \int d q_{z} \tilde{G}_{\Psi Q}\left(q_{z}\right) \exp \left(i q_{z} z\right) ; \\
G_{Q Q}(z)= & \int d x \int d y<\hat{Q}_{z z}^{(B)}(\mathbf{r}) \hat{Q}_{z z}^{(B)}(0)> \\
& =\frac{1}{2 \pi} \int d q_{z} \tilde{G}_{Q Q}\left(q_{z}\right) \exp \left(i q_{z} z\right) ;
\end{aligned}
$$

Here $Q_{z z}^{(B)}=-Q_{x x}^{(B)}-Q_{y y}^{(B)}$ measures the degree of orientational ordering of the rigid part of the copolymer along the z-axis. We note that as a direct consequence of the isotropy of the system the scattering intensities $(\operatorname{Eqs}(7.11-13)$ can be rewritten in the following general form:

$$
\begin{gathered}
\tilde{G}_{\Psi \Psi}\left(q_{z}\right)=\frac{1}{V}<\hat{\Psi}\left(\mathbf{q}^{*}\right) \hat{\Psi}\left(-\mathbf{q}^{*}\right)>; \\
\tilde{G}_{\Psi Q}\left(q_{z}\right)=\frac{1}{V}<\hat{\Psi}\left(\mathbf{q}^{*}\right) \hat{\mathbf{q}}_{\alpha}^{*} \hat{Q}_{\alpha \beta}^{(B)}\left(\mathbf{q}^{*}\right) \hat{\mathbf{q}}_{\beta}^{*}>; \\
\tilde{G}_{Q Q}\left(q_{z}\right)=\frac{1}{V}<\hat{\mathbf{q}}_{\gamma}^{*} \hat{Q}_{\gamma \delta}^{(B)}\left(\mathbf{q}^{*}\right) \hat{\mathbf{q}}_{\delta}^{*} \hat{\mathbf{q}}_{\alpha}^{*} \hat{Q}_{\alpha \beta}^{(B)}\left(\mathbf{q}^{*}\right) \hat{\mathbf{q}}_{\beta}^{*}>.
\end{gathered}
$$

Here $\mathbf{q}^{*}$ is an arbitrary vector of length $\left|\mathbf{q}^{*}\right|=\left|q_{z}\right| ; \hat{\mathbf{q}}^{*}$ is a unit vector along $\mathbf{q}^{*}$. The summation over repeated indices is implied.

The calculations have been performed in the limit $q_{z} \rightarrow 0, N_{A}, N_{B} \rightarrow \infty$, such that $q_{z} N_{B}=$ const and $f=N_{A} /\left(N_{A}+N_{B}\right)=$ const, and for the following sets of parameters: (1) $\mathrm{f}=0.20, \chi N=19.51, v_{B B} / \chi=0.4$; (2) $\mathrm{f}=0.20, \chi N=19.51$, $v_{B B} / \chi=0 ;(3) \mathrm{f}=0.62, \chi N=11.35, v_{B B} / \chi=3.0 ;(4) \mathrm{f}=0.62, \chi N=11.75, v_{B B} / \chi=$ 0 . In all cases the parameters has been chosen such that the system is close to the stability limits which are: for case (1) $\chi N=19.53$; for case (2) $\chi N=19.56$; for case (3) $\chi N=11.40$; and for case (4) $\chi N=11.79$ (see Fig.25).

In Fig.26 $\tilde{G}_{\Psi \Psi}\left(q_{z}\right)$ and $G_{\Psi \Psi}(z)$ are shown for $\mathrm{f}=0.20$ and $\mathrm{f}=0.62$. In the case of a long rigid part, $\mathrm{f}=0.2$ (Fig.(26a)), there are two peaks at $q_{1}$ and $q_{2}$, whose 
positions are determined by the length of that part i.e. $q_{1} \approx 2 \pi /((1-f) N l)$ and $q_{2} \approx 4 \pi /((1-f) N l)$. In the case of a short rigid part, $\mathrm{f}=0.62$, there is only one peak at $q_{1}(\mathrm{Fig}(26 \mathrm{c}))$. The presence of the second harmonic in Fig(26a) and its absence in Fig(26c) indicates that the domain boundaries are sharper in the system containing longer rigid pieces. This is supported by the plots of the real space correlation functions. For $\mathrm{f}=0.62$ (Fig.(26d)) this function is almost sinusoidal, whereas it has additional structure for $\mathrm{f}=0.20$ (Fig.(26b)) In both cases the length of the rigid part of a copolymer sets the length scale. Finally by comparing the cases of $v_{B B} / \chi \neq 0$ (solid line in Fig.(26)) and $v_{B B} / \chi=0$ (dashed line in Fig.(26)) we note that the general features of these functions are not affected very much by the Maier-Saupe parameter $v_{B B}$.

The nematic structure function, $\tilde{G}_{Q Q}\left(q_{z}\right)$, and its Fourier transform (the nematic order parameter - nematic order parameter correlation function), $G_{Q Q}(z)$, are shown in $\operatorname{Fig}(27)$ for $\mathrm{f}=0.62$. The peak at $q_{z}=0$ reflects the tendency toward nematic order, while that at $q_{z} \approx 2 \pi / f N l$ reflects the tendency to lamellar order. They appear in $G_{Q Q}(z)$ as oscillations due to the nascent lamellar order superimposed on the decaying nematic order. Not surprisingly, the Maier-Saupe interaction strongly affects the nematic signature of this correlation function as comparison with the case $v_{B B} / \chi=0$ clearly shows. For $\mathrm{f}=0.2$ the tendency to nematic order is greatly increased (because the system is closer to the isotropic-nematic instability) so that $\tilde{G}_{Q Q}\left(q_{z}\right)$ is completely dominated by the peak at zero wavevector, and the oscillations due to nascent lamellar order are ignorable on the same scale. For this reason, we have not shown this function or its Fourier transform.

In Fig. (28) the mixed correlation functions $\tilde{G}_{\Psi Q}\left(q_{z}\right)$ and $G_{\Psi Q}(z)$ are shown for $\mathrm{f}=0.20$ and $\mathrm{f}=0.62$. Just as for the density-density correlation function (Fig.(26)), the structure of the density-nematic order parameter correlations is richer for $f=0.20$ 
than for $\mathrm{f}=0.62$. Similarly, these structures do not depend very strongly on the Maier-Saupe parameter. The most interesting observation to be made comes from a comparison of the functions $G_{\Psi \Psi}(z)$ and $G_{\Psi Q}(z)$ for f=0.62 (Figs.(26d,28d)). We see that the nematic order parameter in a given direction and the linear concentration of rigid rods in that direction are almost exactly anticorrelated; i.e. large and positive $Q_{z z}^{(B)}(z)$ occurs in planes normal to z which are rich in the flexible A-part of the copolymer. At first sight, this result may appear to be counter intuitive. One knows, for example, that in a mixture of rigid and flexible homopolymers, a higher density of the rigid polymers is found in the nematic phase. However we are concerned with the isotropic phase, and the effect can be understood as arising from that isotropy. As an example, let us assume that we are at a point in space in which the density of B monomers is greater than that of the A. For simplicity, we will assume that there are B monomers only out to a distance $\mathrm{R}$, and $\mathrm{A}$ monomers from $\mathrm{R}$ to $\mathrm{bR}$, with $b=(1-f)^{-1 / 3}$, so that the relative concentration of A monomers is f. With such a density distribution,

$$
\begin{aligned}
G_{\Psi Q}(z)=\left(\frac{3 V}{4 \pi n(b R)^{3}}\right)\left(\int_{0}^{\sqrt{R^{2}-z^{2}}} d s\right. & 2 \pi s P_{2}\left(\frac{z}{\sqrt{z^{2}+s^{2}}}\right) \\
- & \left.\int_{\sqrt{R^{2}-z^{2}}}^{\sqrt{(b R)^{2}-z^{2}}} d s 2 \pi s P_{2}\left(\frac{z}{\sqrt{z^{2}+s^{2}}}\right)\right) \cdot(
\end{aligned}
$$

Carrying out the integrations and setting $R=N_{B} l / 2=N(1-f) l / 2$, we obtain

$$
\begin{aligned}
\rho_{0} l G_{\Psi Q}(z) & =F\left(z / N_{B} l\right) \\
F(x) & =18 x^{2}\left[\frac{1}{6}-\ln \left(\frac{2 x}{(1-f)^{1 / 3}}\right)\right]+\frac{3}{4}\left(\frac{1}{(1-f)^{2 / 3}}-2\right) .
\end{aligned}
$$

As long as the fraction of flexible monomers is not too large, $f<1-2^{-3 / 2} \approx 0.65$, this function is negative at zero argument, thus reproducing the anticorrelation of density and nematic fluctuations. Further, it has a maximum when the argument 
is approximately $0.36(1-f)^{1 / 3}$. In the above picture, we expect the spacing of spheres, and hence the period of the correlation function, to be $N_{B} l /(1-f)^{1 / 3}$. As the model correlation function is minimum at $z=0$, it should also be minimum at integer multiples of this period with maxima a distance $0.36(1-f)^{1 / 3}$ on either side. There are additional minima at half odd integer multiples of the period. For $\mathrm{f}=0.2$ as in Fig. 28b, this model predicts minima at $z / l N_{B}=0,0.54,1.08,1.62$, 2.16 , etc., and maxima at $z / l N_{B}=0.33,0.75,1.41,1.81$, etc. These numbers are in satisfactory agreement with the figure. The double peak structure which this model, with its sharp density profile, produces requires at least the presence of a second harmonic to approximate it. As we have seen, the system with shorter rigid sections, $\mathrm{f}=0.62$, does not exhibit a second harmonic in its structure function. Thus the above model does not describe the function of Fig. 28d very well at all except to reproduce its periodicity and the important negative value at the origin. The above model should not be emphasized unduly, but it does serve to emphasize that the anticorrelation of density and nematic fluctuations that we have found is reasonable.

The behavior of all correlation functions close to tricritical points is similar for all $\mathrm{f}>0.25$, and in particular to the case $\mathrm{f}=0.62$ presented here in detail. For $\mathrm{f} \approx 0.25$ the correlations functions begin to develop the structure shown here for $\mathrm{f}=0.2$. Finally we note that the locations of the minima and maxima of the correlation functions are relatively independent on the Flory-Huggins parameter. Changing it changes the heights of the peaks, but hardly affects their positions. Thus the main features of the local domain structure are determined by the geometry (rigidity and length) of the copolymer molecule and excluded volume effects. In our calculation, the latter is modelled by assuming incompressibility of the system. 


\section{Rigid-flexible blends}

Here we shall show that within the approach presented in this paper we can also obtain the general features of the nematic phase in the system containing rigid and flexible main-chain nematogenic polymers. The other systems like the mixtures of two different rigid polymers, and a system of n-block copolymer consisting of $n$ rigid and flexible parts are described elsewhere ${ }^{60)}$. The Landau-Ginzburg expansion of the free energy is derived, and we show that the general feature of the isotropic nematic phase transition in the system of short flexible polymers and long rigid polymers are qualitatively the same as obtained in the completely different treatment of the density functional theory ${ }^{95,96)}$ applied to infinitely long rods in the solvent. The latter model is commonly known as the Onsager model ${ }^{97}$ ) The detailed study of the (extremely rich) phase diagrams produced in the Landau-Ginzburg model and its variants is contained in $\operatorname{Ref}[57,98-100]$.

In order to describe the system we shall use two order parameters: the usual $\Psi$ parameter and a nematic order parameter $Q_{B}=Q_{z z}^{(B)}$. The nematic order parameter for the flexible part, $S_{A}=Q_{z z}^{(A)}$, can be neglected for the reasons already stated in the previous section and in $\operatorname{Ref}[60]$.

The Landau-Ginzburg free energy for the rigid flexible mixture (with the fraction of the monomers in the flexible polymers being $\bar{\phi}$, can be easily calculated using the approach presented in Appendix A and B. We find the following form of the free energy per monomer:

$$
\Omega\left(Q_{B}, \Psi\right)=\Omega_{I}(\Psi)+\Omega_{B}\left(Q_{B}\right)+\Omega_{C}\left(Q_{B}, \Psi\right) .
$$

$\Omega_{I}$ is given by $\mathrm{Eq}(3.5)$;

$\Omega_{B}\left(Q_{B}\right)=\left(\frac{5}{2 N_{B}(1-\bar{\phi})}-\frac{v_{B B}}{2}\right) Q_{B}^{2}-\frac{25}{21 N_{B}(1-\bar{\phi})^{2}} Q_{B}^{3}+\frac{425}{196 N_{B}(1-\bar{\phi})^{3}} Q_{B}^{4}$. 
is the part of the free energy coming form the nematic ordering of the rigid polymers;

$$
\Omega_{C}\left(Q_{B}, \Psi\right)=\frac{5}{2 N_{B}(1-\bar{\phi})^{2}} Q_{B}^{2} \Psi-\frac{50}{21 N_{B}(1-\bar{\phi})^{3}} Q_{B}^{3} \Psi+\frac{5}{2 N_{B}(1-\bar{\phi})^{3}} Q_{B}^{2} \Psi^{2}
$$

is the term, representing the coupling between the order parameters in the mixture.

Given the free energy $\Omega\left(Q_{B}, \Psi\right)$, one easily finds the conditions for thermal equilibrium of two or more coexisting phases. The condition

$$
\frac{\partial \Omega\left(Q_{B}, \Psi\right)}{\partial Q_{B}}=0
$$

must be satisfied in the absence of external field in all coexisting phases. Additionally the function

$$
G\left(Q_{B}, \Psi\right) \equiv \Omega\left(Q_{B}, \Psi\right)-\frac{\partial \Omega\left(Q_{B}, \Psi\right)}{\partial \Psi} \Psi
$$

must be equal in all phases.

For the two phase coexistance the equations are solved for the three unknowns, $Q_{B}^{(1)}, Q_{B}^{(2)}$, and $\Psi$, i.e. the values of the order parameters in the two phases. In the isotropic phase $Q_{B}=0$, but as we shall we we have the cases when two nematic phases of different $Q_{B}$ can coexist. Please note that at coexistance we have in one phase $\Psi=0$. In the other phase $\Psi$ is equal to the difference in the composition of the coexisting phases.

We consider the mixture of flexible $A$ and rigid $B$ polymers in which the latter are much longer than the former, $N_{B} \gg N_{A}$. At high temperatures one finds that the composition difference between the B-rich nematic phase and A-rich isotropic phase is extremely small, namely

$$
\bar{\phi}_{N}-\bar{\phi}_{I} \sim \frac{N_{A}}{N_{B}} \ll 1
$$

where $\bar{\phi}_{I}\left(\bar{\phi}_{N}\right)$ is the concentration of A monomers in the isotropic (nematic) phase at coexistence. Neglecting terms of the order of $N_{A} / N_{B}$ or smaller, the two-phase 
coexistence region shrinks to a line given by

$$
1 \approx 0.21 v_{B B}^{I N}(1-\bar{\phi}) N_{B} .
$$

where $v_{B B}^{I N}$ is the interaction parameter at the isotropic nematic phase transition. Please note that the parameter is rescaled by $k_{B} T$. The nematic order parameter jumps from zero in the isotropic phase to $Q_{B} \approx 0.275(1-\bar{\phi})$ in the nematic phase. Here $\bar{\phi}$ is the average concentration of A monomers in the mixture at N-I coexistence. Recalling that $Q_{B}$ is the nematic order parameter of the $\mathrm{B}$ monomers divided by the number of all monomers, we see from this result that the jump in nematic order parameter per B monomer is constant, independent of the concentration of the A component. These results are similar in spirit to the results obtained by Warner and Flory ${ }^{101)}$ and Onsager ${ }^{97)}$ for the system of rods diluted by a small particle solvent. If we assume that the monomers in the rigid polymer interact only via excluded volume interactions we can write $v_{B B} \sim \rho_{0} v_{0}$ and $\rho_{0}(1-\bar{\phi})=\rho_{B} N_{B}$, where $\rho_{B}$ is the density of rigid polymers and $v_{0}$ is proportional to the excluded volume of the two monomers. Since $v_{0} \sim l^{2} D_{B}$, where $l$ is the length of the monomer, $L_{B}=l N_{B}$ is the length of the rigid polymer and $D_{B}$ is the thickness of the polymer we finally find the famous Onsager result that in the limit of the rigid rod much larger than the solvent (either monomeric or polymeric) and $L_{B} \gg D_{B}$, the rod density at the isotropic-nematic transition is inversly proportional to $L_{B}^{2}$ i.e.:

$$
\rho_{B} L_{B}^{2} D_{B} \sim 1
$$

Since the Onsager scaling is exact, $\operatorname{Eqs}(8.7-8)$ prove that the method applied here to rigid polymers is correct at the fundamental level.

We also find the phase separation inside the nematic phase. This phase separation is goverened by the Flory Huggins interaction parameter. The location of 
the critical point is given in the limit of $N_{B} \gg N_{A}$ by ( $\operatorname{see} \operatorname{Eq}(3.6,3.7)$ :

$$
\chi_{c}^{m f}=\frac{1}{2 N_{A}}, \quad \bar{\phi}_{c}^{m f}=1-\sqrt{\left(\frac{N_{A}}{N_{B}}\right)}
$$

As is evident from Eq.(8.9) the nematic ordering does not change the critical point temperature, which is the same as in the ordinary polymer blend discussed in section III.

In this paper we have applied the Landau-Ginzburg model to several systems: the polymer blends, diblock and multiblock copolymers, rigid and flexible polymers and their mixtures. The Landau-Ginzburg free energies and scattering intensities were calculated from the Edwards mesoscopic hamiltonian using the field theory methods. We hope that this review will be helpful for the future studies of polymer melts and blends.

\section{Acknowledgements}

This work was supported by two grants from the Komitet Badań Naukowych and Fundacja Współpracy Polsko-Niemieckiej. R.H. acknowledges with appreciation the hospitality and support of the Max-Planck Institute for Polymer Science in Mainz, where the large part of this review has been prepared. We would like

to thank Anne Bohle, Michael Brereton, Wojciech Góźdź, Andrzej Poniewierski, Michael Schick and Andrea Weyersberg for fruitful collaboration and discussions. 


\section{Appendix A: The cumulant expansion}

The method of cumulant expansion is applied here to the binary mixture of A,B homopolymers, but can be easily generalized to any other polymer system. The partition function, $Z\left[\phi_{\gamma}^{\left(n_{\gamma}\right)}\right]$ as a functional of prescribed Fourier transform of the concentration distribution, $\phi_{\gamma}^{\left(n_{\gamma}\right)}(\mathbf{q}),(\gamma=A, B)$ is equal to

$$
Z\left[\phi_{\gamma}^{\left(n_{\gamma}\right)}\right]=\exp \left(-H\left[\phi_{\gamma}^{\left(n_{\gamma}\right)}\right] / k_{B} T\right)\left\langle\prod_{\gamma=A, B} \delta\left(\phi_{\gamma}^{\left(n_{\gamma}\right)}(\mathbf{q})-\hat{\phi}_{\gamma}^{\left(n_{\gamma}\right)}(\mathbf{q})\right\rangle_{0}\right.
$$

where the average over the conformations of the chains is

$$
<\cdots>_{0}=\prod_{\alpha=1}^{n_{A}} \prod_{\beta=1}^{n_{B}} \int D \mathbf{r}^{\alpha} D \mathbf{r}^{\beta} \cdots W_{A}\left[\mathbf{r}^{\alpha}\right] W_{B}\left[\mathbf{r}^{\beta}\right] .
$$

and the interaction hamiltonian, $H$, (see section II) is

$$
\begin{aligned}
H\left[\phi_{\gamma}^{\left(n_{\gamma}\right)}\right] & =k_{B} T \rho_{0} \int \frac{d \mathbf{q}}{(2 \pi)^{3}}\left(\frac{1}{2} w_{A A}\left|\phi_{A}^{\left(n_{A}\right)}(\mathbf{q})\right|^{2}\right. \\
& \left.\left.+\frac{1}{2} w_{B B}\left|\phi_{B}^{\left(n_{B}\right)}(\mathbf{q})\right|^{2}+w_{A B} \phi_{A}^{\left(n_{A}\right)}(\mathbf{q}) \phi_{B}^{\left(n_{B}\right)}(-\mathbf{q})\right)\right) .
\end{aligned}
$$

Now, we can introduce the integral representation of the delta function in Eq.(A.2)

$$
\begin{aligned}
& \prod_{\gamma} \delta\left(\phi_{\gamma}^{\left(n_{\gamma}\right)}(\mathbf{q})-\hat{\phi}_{\gamma}^{\left(n_{\gamma}\right)}(\mathbf{q})\right)= \\
& \quad \prod_{\gamma} \int D J_{\gamma} \exp \left(i \int \frac{d \mathbf{q}}{(2 \pi)^{3}}\left(\phi_{\gamma}^{\left(n_{\gamma}\right)}(\mathbf{q})-\hat{\phi}_{\gamma}^{\left(n_{\gamma}\right)}(\mathbf{q})\right) J_{\gamma}(-\mathbf{q})\right)
\end{aligned}
$$

and expand the exponential in $\hat{\phi}_{\gamma}^{\left(n_{\gamma}\right)}$. Eq.(A.1) now reads

$$
\begin{aligned}
Z\left[\phi_{\gamma}^{\left(n_{\gamma}\right)}\right] & =\exp \left(-H_{I}\left[\phi_{\gamma}^{\left(n_{\gamma}\right)}\right] / k_{B} T\right) \prod_{\gamma=A, B}\left(\int D J_{\gamma} \exp \left(i \int \frac{d \mathbf{q}}{(2 \pi)^{3}} \phi_{\gamma}^{\left(n_{\gamma}\right)}(\mathbf{q}) J_{\gamma}(-\mathbf{q})\right)\right. \\
& \left.\times \sum_{n=0}^{\infty} \frac{(-i)^{n}}{n !} \int \frac{d \mathbf{q}_{1}}{(2 \pi)^{3}} \cdots \int \frac{d \mathbf{q}_{n}}{(2 \pi)^{3}} S_{n}^{*(\gamma)}\left(\mathbf{q}_{1} \cdots \mathbf{q}_{n}\right) J_{\gamma}\left(-\mathbf{q}_{1}\right) \cdots J_{\gamma}\left(-\mathbf{q}_{n}\right)\right)
\end{aligned}
$$


where $S_{n}^{*(\gamma)}$ denotes the n-th moment

$$
S_{n}^{*(\gamma)}\left(\mathbf{q}_{1} \cdots \mathbf{q}_{n}\right)=\left\langle\hat{\phi}_{\gamma}^{\left(n_{\gamma}\right)}\left(\mathbf{q}_{1}\right) \cdots \hat{\phi}_{\gamma}^{\left(n_{\gamma}\right)}\left(\mathbf{q}_{n}\right)\right\rangle_{0}
$$

It is obvious that ideal averages over A chain and over B chain can be performed independently, thus there are no mixed moments. The mixed moments are found only if the chains are connected inside a molecule. This is the case of the diblock copolymer system.

The logarithm of the moments expansion is the cumulant expansion ${ }^{7,14)}$. Thus the second term in Eq.(A.5) can be rewritten as

$$
\prod_{\gamma=A, B} \int D J_{\gamma} \exp \left(F_{\gamma}^{\left(n_{\gamma}\right)}\left[J_{\gamma}\right]+i \int \frac{d \mathbf{q}}{(2 \pi)^{3}} \phi_{\gamma}^{\left(n_{\gamma}\right)}(\mathbf{q}) J_{\gamma}(-\mathbf{q})\right)
$$

where the cumulant expansion, $F_{\gamma}^{\left(n_{\gamma}\right)}\left[J_{\gamma}\right]$, which is a functional of the coupling fields, $J_{\gamma}$, is given by

$$
\begin{aligned}
F_{\gamma}^{\left(n_{\gamma}\right)}\left[J_{\alpha}\right]= & \sum_{n=1}^{\infty} \frac{(-i)^{n}}{n !} \int \frac{d \mathbf{q}_{1}}{(2 \pi)^{3}} \cdots \int \frac{d \mathbf{q}_{n}}{(2 \pi)^{3}} S_{n}^{(\gamma)}\left(\mathbf{q}_{1} \cdots \mathbf{q}_{n}\right) \\
& \delta\left(\mathbf{q}_{1}+\cdots+\mathbf{q}_{n}\right) J_{\gamma}\left(-\mathbf{q}_{1}\right) \cdots J_{\gamma}\left(-\mathbf{q}_{n}\right) .
\end{aligned}
$$

It is easy to represent cumulants in terms of the moments ${ }^{14,22}$ e.g.

$$
\delta\left(\mathbf{q}_{1}+\mathbf{q}_{2}\right) S_{2}^{(A)}\left(\mathbf{q}_{1}, \mathbf{q}_{2}\right)=S_{2}^{*(A)}\left(\mathbf{q}_{1}, \mathbf{q}_{2}\right)-S_{1}^{*(A)}\left(\mathbf{q}_{1}\right) S_{1}^{*(A)}\left(\mathbf{q}_{2}\right)
$$

It is also easy to compute the moments and cumulants explicitely, e.g.

$$
\begin{aligned}
& \frac{1}{V}<\hat{\phi}_{A}^{\left(n_{A}\right)}(\mathbf{q}) \hat{\phi}_{A}^{\left(n_{A}\right)}(-\mathbf{q})>_{0}= \\
& \frac{n_{A} N_{A}}{\rho_{0} V}\left(\frac{1}{\rho_{0} N_{A}}\right)\left(\frac{\left(1+a_{A}\right)}{\left(1-a_{A}\right)} N_{A}-\frac{2 a_{A}}{\left(1-a_{A}\right)^{2}}\left(1-a_{A}^{N_{A}}\right)\right)
\end{aligned}
$$

where $a_{A}=\sin q l_{A} / q l_{A}$ and $q \neq 0$. Expanding this expression in $q l_{A}$ we find

$$
\frac{1}{V}<\hat{\phi}_{A}^{\left(n_{A}\right)}(\mathbf{q}) \hat{\phi}_{A}^{\left(n_{A}\right)}(-\mathbf{q})>_{0}=\frac{n_{A} N_{A}}{\rho_{0} V} \frac{N_{A}}{\rho_{0}} g\left(x_{A}\right)+N_{A} \mathrm{O}\left(1 / N_{A}\right)
$$


where $\bar{\phi}=n_{A} N_{A} / \rho_{0} V$ is the average concentration of $\mathrm{A}$ monomers in the system, $x_{A}=N_{A}\left(q l_{A}\right)^{2} / 6$ and $g(x)=2(x+\exp (-x)-1) / x^{2}$ is the Debye function. Since we are interested in $1 / \sqrt{N_{\gamma}}$ corrections we can neglect all higher order corrections (e.g.1/ $N_{A}$ corrections in $\left.\operatorname{Eq}(\mathrm{A} .9)\right)$. To this point, no approximations have been made other than that which enabled us to express the conditional partition function in the form of Eq.(A.1)

\section{Appendix B: The saddle point approximation and RPA}

Now the integral in expression (A.7) is calculated by the method of the steepest decent, i.e. we approximate (A.7) as follows.

$$
\begin{gathered}
\int D J_{\gamma} \exp \left(F_{\gamma}^{\left(n_{\gamma}\right)}\left[J_{\gamma}\right]+i \int \frac{d \mathbf{q}}{(2 \pi)^{3}} \phi_{\gamma}^{\left(n_{\gamma}\right)}(\mathbf{q}) J_{\gamma}(-\mathbf{q})\right) \approx \\
\exp \left(F_{\gamma}^{\left(n_{\gamma}\right)}\left[J_{\gamma}^{*}\right]+i \int \frac{d \mathbf{q}}{(2 \pi)^{3}} \phi_{\gamma}^{\left(n_{\gamma}\right)}(\mathbf{q}) J_{\gamma}^{*}(-\mathbf{q})\right)
\end{gathered}
$$

Here, at the saddle point, the coupling field, $J_{\gamma}^{*}(\mathbf{q})$, satisfies the equation:

$$
\frac{\delta F_{\gamma}^{\left(n_{\gamma}\right)}\left[J_{\gamma}\right]}{\delta J_{\gamma}(-\mathbf{q})}=-i \phi_{\gamma}^{\left(n_{\gamma}\right)}(\mathbf{q})
$$

Thus, to within an uninteresting constant, the expression (A.7) is approximated by

$$
\prod_{\gamma=A, B} \exp \left(-\bar{F}_{\gamma}^{\left(n_{\gamma}\right)}\left[\Psi_{\gamma}\right]\right)=\prod_{\gamma=A, B} \exp \left(F_{\gamma}^{\left(n_{\gamma}\right)}\left[J_{\gamma}^{*}\right]+i \int \frac{d \mathbf{q}}{(2 \pi)^{3}} \phi_{\gamma}^{\left(n_{\gamma}\right)}(\mathbf{q}) J_{\gamma}^{*}(-\mathbf{q})\right)
$$

Note that Eq.(B.3) together with Eq.(B.2) constitute the Lengendre transform. We have $\bar{F}_{\gamma}^{\left(n_{\gamma}\right)}\left[\Psi_{\gamma}\right]$ :

$$
\begin{gathered}
\bar{F}_{\gamma}^{\left(n_{\gamma}\right)}\left[\Psi_{\gamma}\right]=\sum_{n=2}^{\infty} \frac{1}{n !} \int \frac{d \mathbf{q}_{1}}{(2 \pi)^{3}} \cdots \int \frac{d \mathbf{q}_{n}}{(2 \pi)^{3}} \Gamma_{n}^{(\gamma)}\left(\mathbf{q}_{1} \cdots \mathbf{q}_{n}\right) \\
\times \delta\left(\mathbf{q}_{1}+\cdots+\mathbf{q}_{n}\right) \Psi_{\gamma}\left(-\mathbf{q}_{1}\right) \cdots \Psi_{\gamma}\left(-\mathbf{q}_{n}\right)
\end{gathered}
$$

with the coefficients, $\Gamma_{n}^{(\gamma)}$ which are simply expressed in terms of the cumulants ${ }^{7,14,22}$ e.g.

$$
\Gamma_{2}^{(A)}\left(\mathbf{q}_{1}, \mathbf{q}_{2}\right)=1 / S_{2}^{(A)}\left(\mathbf{q}_{1}, \mathbf{q}_{2}\right)
$$




$$
\Gamma_{3}^{(A)}\left(\mathbf{q}_{1}, \mathbf{q}_{2}, \mathbf{q}_{3}\right)=-\frac{S_{3}^{(A)}\left(\mathbf{q}_{1}, \mathbf{q}_{2}, \mathbf{q}_{3}\right)}{S_{2}^{(A)}\left(\mathbf{q}_{1}, \mathbf{q}_{2}\right) S_{2}^{(A)}\left(\mathbf{q}_{2}, \mathbf{q}_{3}\right) S_{2}^{(A)}\left(\mathbf{q}_{1}, \mathbf{q}_{3}\right)} .
$$

The same formulas are obtained for B-chains. The formulas for $\Gamma_{n}^{(\gamma)}$ functions for $n>3$ are rather lengthy. Here we would like to give the abbreviated formulas for $\Gamma_{n}^{(A)}, n=4,5$, needed in our later calculations. They are as follows:

$$
\begin{gathered}
\Gamma_{4}^{(A)}=-\left(S_{4}^{(A)}-3 \int S_{3}^{(A)} \Gamma_{2}^{(A)} S_{3}^{(A)}\right)\left(\Gamma_{2}^{(A)}\right)^{4}, \\
\Gamma_{5}^{(A)}=-\left(S_{5}^{(A)}+15 \int S_{3}^{(A)} \Gamma_{2}^{(A)} \int S_{3}^{(A)} \Gamma_{2}^{(A)} S_{3}^{(A)}-10 \int S_{4}^{(A)} \Gamma_{2}^{(A)} S_{3}^{(A)}\right)\left(\Gamma_{2}^{(A)}\right)^{5},
\end{gathered}
$$

where the proper symmetrization and the integration over dummy variables are assumed.

We note that Eq.(A.1) together with Eq.(B.4) constitute the basis of the random phase approximation $(\mathrm{RPA})^{2,22)}$ and the RPA vertex functions $\Gamma_{n}^{(0)}$ are easily obtained by summing the interaction term form the hamiltonian and the term involving $\Gamma_{n}^{(A)}$ and $\Gamma_{n}^{(B)}$. For example we have

$$
\Gamma_{2}^{(0)}=\Gamma_{n}^{(A)}+\Gamma_{2}^{(B)}-2 \chi \rho_{0}
$$

and

$$
\Gamma_{3}^{(0)}=\Gamma_{3}^{(A)}-\Gamma_{3}^{(B)}
$$

In the limit of $q \rightarrow 0$ we find

$$
\Gamma_{3}^{(A)}(0,0,0)=-\frac{1}{\bar{\phi}} \Gamma_{2}^{(A)}(0,0)
$$

(analogously for $\mathrm{B}$ with $\bar{\phi}$ changed to $1-\bar{\phi})$. The right hand side of $\mathrm{Eq}(\mathrm{B} .11)$ is given by the inverse of $\mathrm{Eq}(\mathrm{A} .9)$ ( see $\mathrm{Eq}(3.9)$ ). 


\section{Appendix C: One-loop equations for the vertex functions}

Fig.7 and Fig.8 show the one-loop diagrams contributing to $\Gamma_{2}$ and $\Gamma_{3}$, respectively. Note that $\Gamma_{4}^{(0)}$ and $\Gamma_{3}^{(0)}$ contribute to $\Gamma_{2}$ (Fig.7), while $\Gamma_{3}^{(0)}$ and $\Gamma_{5}^{(0)}$ contribute to $\Gamma_{3}$ (Fig.8). The solid lines represent $1 / \Gamma_{2}^{(0)}$ while the higher order vertex functions are represented by points in the diagrams. The n-body vertex function has $\mathrm{n}$ lines emanating from the point representing this function. The dashed lines are not parts of the diagrams but are included only for clarity (according to Ref.[7]). The final approximation (Hartree) in the framework of the loop expansion is to make the equations self consistent. Here it corresponds to the change of $\Gamma_{2}^{(0)}$ and $\Gamma_{3}^{(0)}$ appearing in the integrals, represented by the diagrams in Figs. $(7,8)$, to $\Gamma_{2}$ and $\Gamma_{3}$. The final self-consistent one-loop equations for the two-body and three-body vertex functions are as follows:

$$
\begin{gathered}
\Gamma_{2}(\mathbf{q},-\mathbf{q})=\Gamma_{2}^{(0)}(\mathbf{q},-\mathbf{q})+\frac{1}{2} \int \frac{d \mathbf{k}}{(2 \pi)^{3}} \frac{\Gamma_{4}^{(0)}(\mathbf{q},-\mathbf{q}, \mathbf{k},-\mathbf{k})}{\Gamma_{2}(\mathbf{k},-\mathbf{k})} \\
-\frac{1}{2} \int \frac{d \mathbf{k}}{(2 \pi)^{3}} \frac{\left(\Gamma_{3}(\mathbf{q}, \mathbf{k},-\mathbf{q}-\mathbf{k})\right)^{2}}{\Gamma_{2}(\mathbf{k},-\mathbf{k}) \Gamma_{2}(\mathbf{q}+\mathbf{k},-\mathbf{q}-\mathbf{k})}, \\
\Gamma_{3}(\mathbf{q}, \mathbf{p},-\mathbf{q}-\mathbf{p})=\Gamma_{3}^{(0)}(\mathbf{q}, \mathbf{p},-\mathbf{q}-\mathbf{p}) \\
+\int \frac{d \mathbf{k}}{(2 \pi)^{3}} \frac{\Gamma_{3}(\mathbf{q}, \mathbf{k},-\mathbf{q}-\mathbf{k}) \Gamma_{3}(\mathbf{p}, \mathbf{k},-\mathbf{p}-\mathbf{k}) \Gamma_{3}(-\mathbf{q}-\mathbf{p}, \mathbf{p}+\mathbf{k}, \mathbf{q}-\mathbf{k})}{\Gamma_{2}(\mathbf{k},-\mathbf{k}) \Gamma_{2}(\mathbf{q}+\mathbf{k},-\mathbf{q}-\mathbf{k}) \Gamma_{2}(\mathbf{p}-\mathbf{k},-\mathbf{p}+\mathbf{k})} \\
+\frac{1}{2} \int \frac{d \mathbf{k}}{(2 \pi)^{3}} \frac{\Gamma_{5}^{(0)}(\mathbf{k},-\mathbf{k}, \mathbf{q}, \mathbf{p},-\mathbf{q}-\mathbf{p})}{\Gamma_{2}(\mathbf{k},-\mathbf{k})} .
\end{gathered}
$$

Before proceeding, we first want to determine the order (in terms of $N_{\gamma}, \gamma=A, B$ ) of successive terms in Eqs.(C.1,2). For simplicity, we consider for a moment the case of $N_{A}=N_{B}=N$ and $l_{A}=l_{B}=l$. For equal length of $\mathrm{A}$ and $\mathrm{B}$ polymers the critical concentration is $\bar{\phi}_{c}=1 / 2$ and at the critical point only the first and the second terms in Eq.(C.3) are nonzero. By comparing these two terms we find the following relation at the critical point:

$$
\frac{1}{\left|\Gamma_{2}^{(0)}(0,0)\right|} \int \frac{d \mathbf{k}}{(2 \pi)^{3}} \frac{\Gamma_{4}^{(0)}(\mathbf{k},-\mathbf{k}, 0,0)}{\Gamma_{2}(\mathbf{k},-\mathbf{k})} \sim \frac{l}{\Lambda}+N \frac{l^{3}}{\Lambda^{3}}
$$


where $2 \pi / \Lambda$ is the upper cutoff in the integral. As we can see the choice of the cutoff is crucial for determining the relative magnitude of the mean-field value of $\Gamma_{2}$ (that is $\Gamma_{2}^{(0)}$ ) and the first (one-loop) correction to it. There are no ready recipes for the choice of the cutoff. Here we take it proportional to the radius of gyration i.e. to $\sqrt{N}$. The discussion of the cutoff is given in Appendix D.

In the solution of Eq.(C.1,2) we use the following approximation for $\Gamma_{2}^{(A)}$ (similarly for $\Gamma_{2}^{(B)}$ ) (see Appendix A and B):

$$
\Gamma_{2}^{(A)}(\mathbf{q},-\mathbf{q})=\frac{1+R_{A}^{2} q^{2} / 2}{\bar{\phi} N_{A}}
$$

The coefficient (Eq.C4) in front of $q^{2}$ is obtained from the expansion of the expression (A.9) for large $q l_{A}$. Finally we observe that

$$
\begin{gathered}
\Gamma_{3}^{(A)}(\mathbf{q},-\mathbf{q}, 0)=-\Gamma_{2}^{(A)}(\mathbf{q},-\mathbf{q}) / \bar{\phi}, \\
\Gamma_{4}^{(A)}(\mathbf{q},-\mathbf{q}, 0,0)=2 \Gamma_{2}^{(A)}(\mathbf{q},-\mathbf{q}) / \bar{\phi}^{2}, \\
\Gamma_{5}^{(A)}(\mathbf{q},-\mathbf{q}, 0,0,0)=-6 \Gamma_{2}^{(A)}(\mathbf{q},-\mathbf{q}) / \bar{\phi}^{3} .
\end{gathered}
$$

We have analogous equations for B chains with $\bar{\phi}$ changed to $1-\bar{\phi}$.

\section{Appendix D: The upper wavevector cutoff in polymer blends}

As we have seen in section III the fluctuation corrections to the critical temperature strongly depend on the upper wavevector cutoff. Although it is ubiquitous in statistical mechanics there are no ready recipes for the choice of the cutoff. In low molecular mass liquids we usually have one natural length scale, which corresponds to the size of a molecule and the cutoff is usually made proportional to this length scale. In the polymer mixtures the problem is more complicated since we have three different length scales: the total length of a polymer molecule, $\mathrm{Nl}$, the size of the region occupied by a polymer molecule (proportional to the radius of 
gyration), $\sim \sqrt{N} l$, and finally, the microscopic length scale $l$, which is determined by the size of a single monomer. We believe that $\Lambda$ should be proportional to the radius of gyration, that is to $\sqrt{N}$. At high temperatures, where the interactions between the monomers are irrelevant, the monomer-monomer correlation function decays exponentially with the characteristic correlation length which is proportional to the radius of gyration. Moreover, in all our calculations the specific structure of monomers has not been taken into account. The microscopic length scale is irrelevant here. Finally, if we chose the microscopic length scale as the cutoff the fluctuation corrections would survive in the limit of $N \rightarrow \infty$ and thus RPA would not be the correct description in this limit. We believe this is not the case. We note that since the size of the polymer molecule in the blend is roughly proportional to $\sqrt{N}$; this choice is also in accordance with the prescription known from low molecular mass systems where the cutoff is made proportional to the size of a molecule. In the case of assymetric mixture we postulate that the cutoff should be equal to $2 \pi /\left(\Lambda\left(R_{A}, R_{B}\right)\right)$, where $\Lambda$ is the symmetric function of the two radiuses of gyrations for A and B chains. Finally, we assume the scaling form for $\Lambda$ :

$$
\Lambda\left(R_{A}, R_{B}\right)=R_{A} f(x)
$$

where $x=R_{B} / R_{A}$. From the symmetry properties of $\Lambda$, we have the following equation for $f(x)$ :

$$
\frac{1}{x} f(x)=f\left(\frac{1}{x}\right)
$$

The general solution of this functional equation is

$$
f(x)=\sqrt{C_{1} \frac{1+x^{3}}{1+x}+C_{2} x} .
$$

Here $C_{1}$ and $C_{2}$ are two constants which depend on the microscopic details of the system. We cannot determine them from the present theory, thus, in the interpretation of experimental results, they should be the fitting parameters. 
In the simplest approximation we can set $R_{A} \sim \sqrt{N_{A}} l_{A}$. In general in the polymer system this radius changes with temperature and concentration and so it would be desirable here to determine it self consistently from the theory. The equation for the radius of gyration, $R$, offers a simple way for the self consistent determination of the cutoff. if we set it equal to $2 \pi C / R$ where $C$ is some numeric constant. Thus apart from this constant the cutoff would be determined from the theory. In section III D the equations for the radius of gyration are shown to involve the collective structure factor (proportional to the scattering intensity $\mathrm{Eq}(3.9)$ ). In this way the equation for the collective structure factor and the equation for the radius of gyration are mutually coupled.

\section{Appendix E}

Here we present the results used in section IIIC for the calculation of the radius of gyration. Here we shall follow the equations given in Ref[20]. For the fourth order correlation function we have:

$$
\begin{aligned}
& <\hat{\phi}_{A}^{(1)}(\mathbf{q}) \hat{\phi}_{A}^{(1)}(-\mathbf{q}) \hat{\phi}_{A}^{(1)}(\mathbf{k}) \hat{\phi}_{A}^{(1)}(-\mathbf{k})>_{0}=8 g_{1}(q, 0, k)+2\left(g_{1}(q, \mathbf{q}+\mathbf{k}, q)\right. \\
& \left.+g_{1}(q, \mathbf{q}-\mathbf{k}, q)+g_{1}(k, \mathbf{q}+\mathbf{k}, k)+g_{1}(k, \mathbf{q}-\mathbf{k}, k)\right) \\
& +4\left(g_{1}(q, \mathbf{q}+\mathbf{k}, k)+g_{1}(q, \mathbf{q}-\mathbf{k}, k)\right) .
\end{aligned}
$$

Here

$$
\begin{aligned}
& g_{1}\left(q_{1}, q_{2}, q_{3}\right)=\frac{N_{A}}{x_{1} x_{2} x_{3}}+\frac{p\left(x_{1}\right)}{x_{1}\left(x_{1}-x_{2}\right)\left(x_{1}-x_{3}\right)} \\
& +\frac{p\left(x_{2}\right)}{x_{2}\left(x_{2}-x_{3}\right)\left(x_{2}-x_{1}\right)}+\frac{p\left(x_{3}\right)}{x_{3}\left(x_{3}-x_{2}\right)\left(x_{3}-x_{1}\right)}
\end{aligned}
$$

where $x_{i}=q_{i}^{2} l^{2} / 6$ and $p(x)=\left(\exp \left(-x N_{A}\right)-1\right) / x$. For the two point correlation function we find

$$
<\hat{\phi}_{A}^{(1)}(\mathbf{q}) \hat{\phi}_{A}^{(1)}(-\mathbf{q})>_{0}=2\left(N_{A}+p(x)\right) / x
$$

and $x=q^{2} l^{2} / 6$. 
In order to obtain the radius of gyration one has to differentiate

$$
\begin{aligned}
h(\mathbf{k}, \mathbf{q}) & =<\hat{\phi}_{A}^{(1)}(\mathbf{q}) \hat{\phi}_{A}^{(1)}(-\mathbf{q}) \hat{\phi}_{A}^{(1)}(\mathbf{k}) \hat{\phi}_{A}^{(1)}(-\mathbf{k})>_{0} \\
& -<\hat{\phi}_{A}^{(1)}(\mathbf{q}) \hat{\phi}_{A}^{(1)}(-\mathbf{q})>_{0}<\hat{\phi}_{A}^{(1)}(\mathbf{k}) \hat{\phi}_{A}^{(1)}(-\mathbf{k})>_{0}
\end{aligned}
$$

twice with respect to $q$ and finally take the limit of $q \rightarrow 0$. We find

$$
\begin{aligned}
h^{\prime \prime}(\mathbf{k}, 0) & =f(\mathbf{k})=4 N_{A}^{5} \cos ^{2} \theta\left(-120+120 \exp (y)-96 y-24 \exp (y) y-24 y^{2}\right. \\
& \left.-12 \exp (y) y^{2}+4 \exp (y) y^{3}+y^{4}\right) /\left(3 \exp (y) y^{5}\right),
\end{aligned}
$$

where $y=N_{A} k^{2} l^{2} / 6$ and $\theta$ is the angle between the $\mathbf{q}$ and $\mathbf{k}$ vector.

\section{Appendix F: One-loop equation for $\Gamma_{2}$ in the lamellar phase}

In the lamellar phase of the diblock copolymer system the field $\Psi$ is approximated by the single harmonic:

$$
\Psi=A \cos \left(q_{l} z\right)
$$

In general $q_{l}$, which describes the periodicity of the phase, is different from the $q^{*}$, which marks the divergence of the structure factor. In practice they are very close. The vertex function in this case, $\Gamma_{2}(\mathbf{q}, A)$ is the function of the amplitude $A$ and the wavevector q. In the disordered phase $\mathrm{A}=0$, and $\Gamma_{2}$ depends only on the modulus of q. The equation for the vertex function for the symmetric case $(f=1 / 2)$ is as follows ${ }^{20)}$ :

$$
\begin{aligned}
& \Gamma_{2}(\mathbf{q}, A)=\Gamma_{2}^{(0)}(|\mathbf{q}|, A=0)+\frac{1}{2} \int \frac{d \mathbf{k}}{(2 \pi)^{3}} \Gamma_{4}^{(0)}(\mathbf{q},-\mathbf{q}, \mathbf{k},-\mathbf{k}) \Gamma_{2}^{-1}(\mathbf{k}, A) \\
& +A^{2} \Gamma_{4}^{(0)}\left(\mathbf{q},-\mathbf{q}, q_{l},-q_{l}\right)
\end{aligned}
$$

Please note that the function, $\Gamma_{2}$ is not isotropic in the lamellar phase, because a particular direction ( $\mathrm{z}$ in this case $(\mathrm{Eq}(\mathrm{F} .1))$ ) has been selected. The dependence of $\Gamma_{4}^{(0)}$ on angles is rather weak. 


\section{Appendix $G$}

The coefficent in the Landau-Ginzburg free energy Eqs(6.26-27) are as follows:

$$
\begin{aligned}
& a=\gamma_{1} \\
& b=\frac{2 \gamma_{2} \gamma_{3}-\gamma_{4}^{2}}{4 \gamma_{3}} \\
& c=\frac{4 \gamma_{3}^{3} \gamma_{5}-3 \gamma_{3}^{2} \gamma_{4} \gamma_{8}+3 \gamma_{3} \gamma_{4}^{2} \gamma_{6}-\gamma_{4}^{3} \gamma_{7}}{12 \gamma_{3}^{3}} .
\end{aligned}
$$

where

$$
\begin{aligned}
& \gamma_{1}=\frac{1}{(1-\phi)}-\frac{\chi N}{2} \\
& \gamma_{2}=\frac{1}{3(1-\phi)^{3}} \\
& \gamma_{3}=\frac{1}{\phi(1-\phi)} \\
& \gamma_{4}=\frac{1}{(1-\phi)^{2}} \\
& \gamma_{5}=\frac{1}{5(1-\phi)^{5}} \\
& \gamma_{6}=\frac{1}{(1-\phi)^{3}} \\
& \gamma_{7}=-\frac{(1-2 \phi)}{2(1-\phi)^{2} \phi^{2}} \\
& \gamma_{8}=\frac{1}{(1-\phi)^{4}} .
\end{aligned}
$$

Note that with the exception of $\gamma_{1}$, all coefficients are determined solely by the entropy of mixing.

The consolute line is given by the vanishing of the coefficient $a$ in Eq. or, from Eqs. (G.1) and (G.2),

$$
\chi N=\frac{2}{(1-\phi)} .
$$

A tricritical point arises when the coefficients $a$ and $b$ vanish simultaneously, which occurs at the point

$$
\chi_{t r i} N=6,
$$




$$
\phi_{t r i}=2 / 3 .
$$

We have established, therefore, that this point, the point at which the disorder line and Lifshitz lines all meet, is a tricritical point. To establish that it is a Lifshitz tricritical point we have to check $g_{0}$. Here we have

$$
\begin{gathered}
g_{0}=\frac{1}{3}\left(\frac{1}{(1-\phi)}-\frac{(\chi N)^{2} \phi}{8}\right) \frac{N l^{2}}{6} \\
h_{0}=\frac{1}{36}\left(\frac{1}{(1-\phi)}+\frac{9(\chi N)^{2} \phi}{16}-\frac{(\chi N)^{3} \phi^{2}}{8}\right) \frac{N^{2} l^{4}}{36} .
\end{gathered}
$$

We immediately verify that at the tricritical point $N \chi_{t r i}=6, \phi_{t r i}=2 / 3$ at which the coefficients $a(\chi, \phi)$ and $b(\chi, \phi)$ both vanish, the coefficient $g_{0}(\chi, \phi)$ also vanishes. Therefore the point is a Lifshitz tricritical point as stated (see also Appendix H).

\section{Appendix H: Influence of Lifshitz Tricritical Point}

At values of $\chi$ larger than those given by the consolute line (in section VI), the system separates into A-rich and B-rich phases (Fig.23). We shall calculate approximately the surface energy between these two phases to illustrate the effect of the proximity of the Lifshitz tricritical point. The two coexisting phases are characterized by uniform order parameters of amplitude $\pm \eta_{0}$, where this amplitude is obtained by minimizing the free energy of Eq. (6.26) and requiring a uniform solution. One obtains

$$
\eta_{0}^{2}=\frac{-b+\sqrt{b^{2}-3 a c}}{3 c} .
$$

To determine the interfacial profile $\eta(z)$ between these phases, we require a nonuniform solution of the Euler-Lagrange equation which expresses the minimization of the free energy Eq. (6.28). The solution must also satisfy the boundary conditions

$$
\eta( \pm \infty)= \pm \eta_{0} .
$$


The Euler-Lagrange equation is of fourth order and nonlinear, and we are unable to solve it. In order to obtain an analytic form for the surface free energy, we approximate the bulk part of the free energy by a double parabola with the same curvature at the minima;

$$
\frac{1}{2}\left(-|a| \eta^{2}(\mathbf{r})+b \eta^{4}(\mathbf{r})+c \eta^{6}(\mathbf{r})\right) \approx \begin{cases}2|a| y(s)\left(\eta(\mathbf{r})-\eta_{0}\right)^{2} & \text { if } \eta>0 \\ 2|a| y(s)\left(\eta(\mathbf{r})+\eta_{0}\right)^{2} & \text { if } \eta<0\end{cases}
$$

where

$$
y(s) \equiv\left[\left(1+s^{2}\right)^{1 / 2}-s\right]\left(1+s^{2}\right)^{1 / 2}
$$

and

$$
s \equiv b /(3|a| c)^{1 / 2}
$$

The parameter $s$ is a scaling field, a dimensionless measure of the deviation, $b$, of the system from tricritical behavior measured with respect to $a^{1 / 2}$, where $a$ is proportional to $t \equiv\left(T-T_{\text {con }}\right) / T_{\text {con }}$, the deviation of the temperature from the consolute temperature. With this approximation in the free energy, the EulerLagrange equation is piecewise linear

$$
h_{0} \frac{d^{4} \eta}{d z^{4}}-g_{0} \frac{d^{2} \eta}{d z^{2}}+4|a| y(s)\left(\eta \pm \eta_{0}\right)=0
$$

The form of the solution depends upon the ratio $y(s) / p^{2}$ where $p$ is a second scaling field

$$
p \equiv g_{0} /\left(4|a| h_{0}\right)^{1 / 2}
$$

a dimensionless measure of the amplitude of the square gradient term in the free energy. It is a measure of the deviation of the system from Lifshitz behavior. The ratio of $y / p^{2}$ is easy to interpret; the scaling field $p$ for fixed $g_{0}$ diverges as the consolute line is approached. Similarly, the scaling field $s$ for fixed $b$ also diverges as the consolute line is approached. However, the function $y(s)$, only varies by a factor 
of two as $s$ varies from zero to infinity. Thus $y / p^{2}$ will always be smaller than unity sufficiently close to the consolute line; how close in terms of temperature depends on the amplitude $g_{0}$, that is, how close the system is to Lifshitz behavior.

In general the form of the profile is

$$
\eta(z)= \begin{cases}-\eta_{0}+K_{1} \exp \left(z / \xi_{+}\right)+K_{2} \exp \left(z / \xi_{-}\right), & \text {if } z<0 \\ \eta_{0}-K_{1} \exp \left(-z / \xi_{+}\right)-K_{2} \exp \left(-z / \xi_{-}\right), & \text {if } z>0\end{cases}
$$

with

$$
\begin{gathered}
K_{1}=\frac{\eta_{0}\left(\xi_{+} / \xi_{-}\right)^{2}}{\left(\xi_{+} / \xi_{-}\right)^{2}-1}, \\
K_{2}=-\frac{\eta_{0}}{\left(\xi_{+} / \xi_{-}\right)^{2}-1},
\end{gathered}
$$

and two decay length in the system

$$
\xi_{ \pm}^{-2}=2\left(\frac{|a|}{h_{0}}\right)^{1 / 2} p\left(1 \pm\left(1-\frac{y}{p^{2}}\right)^{1 / 2}\right)
$$

As the consolute line is approached, the correlation length $\xi_{-}$diverges as $t^{-1 / 2}$, while $\xi_{+}$is finite unless the system approaches the Lifshitz tricritical (or critical) point. It is easy to see that for $y / p^{2}<1, \xi_{ \pm}$are real and the profiles are monotonically decaying functions at large distances from the interface. For $y / p^{2}>1$, that is, sufficiently close to the Lifshitz tricritical point, $\xi_{ \pm}$are complex and the profiles are no longer monotonically decaying, but are damped oscillatory functions. Using $\mathrm{Eq}(\mathrm{H} .8-\mathrm{H} .11)$ this oscillatory profile can be written in the following form:

$$
\eta(z)= \begin{cases}-\eta_{0}+\exp (z / \xi)\left(\eta_{0} \cos (2 \pi z / \lambda)+K_{3} \sin (2 \pi z / \lambda)\right) & \text { if } z<0 \\ \eta_{0}+\exp (-z / \xi)\left(-\eta_{0} \cos (2 \pi z / \lambda)+K_{3} \sin (2 \pi z / \lambda)\right) & \text { if } z>0\end{cases}
$$

where

$$
\begin{gathered}
K_{3}=\frac{1}{\sqrt{y / p^{2}-1}} \\
\xi^{-2}=\left(\frac{|a|}{h_{0}}\right)^{1 / 2} p\left(1+\frac{y^{1 / 2}}{p}\right),
\end{gathered}
$$


and

$$
\left(\frac{2 \pi}{\lambda}\right)^{2}=\left(\frac{|a|}{h_{0}}\right)^{1 / 2} p\left(1-\frac{y^{1 / 2}}{p}\right),
$$

Note that this profile has the similar form as the correlation function of the disordered phase on the copolymer-rich side of the disorder line (section VI B). We note here that the non-monotonic profiles are encountered in homopolymer-rich phases sufficiently close to the Lifshitz tricritical point. The correlation length $\xi$ diverges as the Lifshitz tricritical point is approached as $t^{-1 / 4}$, as expected from Sec.VI C.

The interfacial tension is obtained by inserting these profiles into Eq. (6.26) and dividing by the area of the planar interface. The result can be written in the scaling form

$$
\sigma\left(t, b, g_{0}\right)=\left(\frac{|a|^{5} h_{0}}{9 c^{2}}\right)^{1 / 4} \frac{2 \rho_{0} k_{B} T}{N} \Sigma(s, p)
$$

where

$$
\Sigma(s, p)= \begin{cases}\frac{y^{3 / 2}}{\left(1+s^{2}\right)^{1 / 2}} \frac{\left(2 p+y^{1 / 2}\right)}{\left[p-\left(p^{2}-y\right)^{1 / 2}\right]^{1 / 2}+\left[p+\left(p^{2}-y\right)^{1 / 2}\right]^{1 / 2}}, & \text { if } y \leq p^{2} \\ \frac{y^{3 / 2}}{\left(1+s^{2}\right)^{1 / 2}} \frac{\left(2 p+y^{1 / 2}\right)}{\left(p+y^{1 / 2}\right)^{1 / 2}} & \text { if } y \geq p^{2} .\end{cases}
$$

The surface tension is proportional to $N^{-1 / 2}$ as $h_{0}$ is proportional to $N^{2}$. This expression for the surface tension has the following properties; on approaching the Lifshitz tricritical point, $t \rightarrow 0, \mathrm{p}$ and s constant,

$$
\sigma(t, s, p) \sim t^{5 / 4}
$$

on approaching an ordinary tricritical point, $t \rightarrow 0$, s constant, $p \sim t^{-1 / 2}$,

$$
\sigma(t, s, p) \sim t
$$

on approaching a Lifshitz critical point, $t \rightarrow 0, s \sim t^{-1 / 2}$, p constant,

$$
\sigma(t, s, p) \sim t^{7 / 4}
$$


on approaching an ordinary critical point, $t \rightarrow 0, \mathrm{~s} / \mathrm{p}$ constant, $s \sim t^{-1 / 2}$,

$$
\sigma(t, s, p) \sim t^{3 / 2}
$$

All of these behaviors are the ones expected in mean field theory. The crossover between these behaviors is governed by the two scaling fields $s$ and $p$ of Eqs. (H.5) and (H.7).

Sufficiently near a transition so that fluctuations become important, the surface tension will still have a scaling form

$$
\sigma\left(t, b, g_{0}\right)=t^{\mu} \Sigma\left(\frac{b}{t^{1 / \phi_{t}}}, \frac{g_{0}}{t^{1 / \phi_{g}}}\right)
$$

but the form of the scaling function $\Sigma$ will differ from that given above, and the critical exponent $\mu$ and the crossover exponents $\phi_{t}$ and $\phi_{g}$ will differ from their mean-field values $5 / 4,2$, and 2 , respectively. As noted earlier, there are no calculations as to the values these exponents should take.

The presence of the Lifshitz tricritical point also affects the results of scattering measurements as seen in the structure function. At long wavelengths, and sufficiently close to the consolute line, the form of a general structure factor is

$$
I(q)=\left(a+g_{0} q^{2}+K q^{4}\right)^{-1}
$$

where $K$, a function of the copolymer concentration, will depend on the particular structure function. On approaching any point on the consolute line except the Lifshitz tricritical point, it is convenient to rewrite this expression in the form

$$
\begin{aligned}
I(q) & =a^{-1}\left(1+\frac{g_{0}}{a} q^{2}+\frac{K a}{g_{0}^{2}} \frac{g_{0}^{2}}{a^{2}} q^{4}\right)^{-1} \\
& =a^{-1}\left(1+(q \xi)^{2}+K^{\prime} p^{-2}(q \xi)^{4}\right)^{-1}
\end{aligned}
$$

where, to within numerical factors, $\xi$ is the ordinary correlation length $\xi_{-}$of Eq. (H.9). As the scaling field $p$ diverges as the consolute line is approached, the term 
in $q^{4}$ becomes unimportant and the structure function can be written in the scaling form

$$
\begin{aligned}
I(t, q) & =t s(q \xi) \\
s(x) & =\left(1+x^{2}\right)^{-1}, \quad p \rightarrow \infty
\end{aligned}
$$

that is, the usual Lorentzian form. On the other hand, if one approaches the Lifshitz tricritical point so that the scaling field $p$ remains finite, it is more convenient to write Eq. (H.23) as

$$
\begin{aligned}
I(q) & =a^{-1}\left(1+\frac{g_{0}}{(a K)^{1 / 2}}\left(\frac{K}{a}^{1 / 4} q\right)^{2}+\left(\frac{K}{a}^{1 / 4} q\right)^{4}\right)^{-1} \\
& =a^{-1}\left(1+K^{\prime \prime} p(\xi q)^{2}+(\xi q)^{4}\right)^{-1}
\end{aligned}
$$

where $K^{\prime \prime}$ is an unimportant function of the copolymer concentration and $\xi$ is again the correlation function which behaves as $t^{-1 / 4}$ as the Lifshitz tricritical point is approached. Again, this can be expressed in a scaling form

$$
\begin{aligned}
I(t, q) & =t s(q \xi) \\
s(x) & =\left(1+K^{\prime \prime} p x^{2}+x^{4}\right)^{-1}
\end{aligned}
$$

with $p$ fixed. Therefore, the presence of the Lifshitz tricritical behavior makes itself known by the fact that, in a fit of the structure function at long wavelengths, terms of order $q^{4}$ cannot be neglected with respect to terms of order $q^{2}$. Again, when the effects of fluctuations are included, one expects the structure function to take a scaling form

$$
I\left(q, T, b, g_{0}\right)=t^{\gamma} s\left\{q \xi\left(t, \frac{b}{t^{1 / \phi_{t}}}, \frac{g_{0}}{t^{1 / \phi_{g}}}\right\},\right.
$$

but the form of the scaling function will differ, and the critical exponent $\gamma$ and the crossover exponents $\phi_{t}$ and $\phi_{g}$ will differ from their mean-field values of 1 , 
2, and 2. Experiments on mixtures of homopolymers have observed the change in the exponent $\gamma$ from its mean-field value of 1 to its fluctuation driven value of 1.26 as the consolute line was approached (section III). Presumably, a similar change could be observed if the Lifshitz tricritical point were approached, because the region of fluctuation dominated behavior is somewhat larger. Again, such an observation would be most interesting as there exist no calculated value for this exponent characterizing the Lifshitz tricritical point.

\section{Appendix I: The ideal averages for the rigid-flexible diblocks}

Below we show the results of the calculations of the ideal averages. Here $a=$ $\sin (q l) / q l, \mathrm{f}$ is the fraction of A monomers in the copolymer and $\mathrm{N}=\mathrm{N}_{A}+\mathrm{N}_{B}$ is the number of all the monomers in a copolymer. We find,

$$
\begin{aligned}
& \frac{1}{V}<\hat{\phi}_{A}^{(n)}(\mathbf{q}) \hat{\phi}_{A}^{(n)}(-\mathbf{q})>_{0}=\frac{1}{\rho_{0} N}\left(\frac{(1+a)}{(1-a)} f N-\frac{2 a}{(1-a)^{2}}\left(1-a^{f N}\right)\right) . \\
& \frac{1}{V}<\hat{\phi}_{A}^{(n)}(\mathbf{q}) \hat{\phi}_{B}^{(n)}(-\mathbf{q})>_{0}=\frac{1}{\rho_{0} N} \frac{1-a^{f N}}{1-a} \int_{0}^{1} d x\left(\frac{\sin (q l(1-f) N x / 2)}{\sin (q l x / 2)}\right. \\
& \times \cos (q l[(1-f) N+1] x / 2)) \\
& \frac{1}{V}<\hat{\phi}_{B}^{(n)}(\mathbf{q}) \hat{\phi}_{B}^{(n)}(-\mathbf{q})>_{0}=\frac{1}{\rho_{0} N} \int_{0}^{1} d x \frac{\sin ^{2}(q l(1-f) N x / 2)}{\sin ^{2}(q l x / 2)} ; \\
& \frac{1}{V}<\hat{\phi}_{A}^{(n)}(\mathbf{q}) \hat{Q}_{x x}^{(B)}(-\mathbf{q})>_{0}=\frac{1}{V}<\hat{\phi}_{A}^{(n)}(\mathbf{q}) \hat{Q}_{y y}^{(B)}(-\mathbf{q})>_{0}= \\
& \frac{1}{\rho_{0} N} \frac{1-a^{f N}}{1-a} \int_{0}^{1} d x\left(\left(\frac{1}{4}-\frac{3}{4} x^{2}\right) \frac{\sin (q l(1-f) N x / 2)}{\sin (q l x / 2)} \cos (q l[(1-f) N+1] x / 2)\right) \\
& \frac{1}{V}<\hat{\phi}_{B}^{(n)}(\mathbf{q}) \hat{Q}_{x x}^{(B)}(-\mathbf{q})>_{0}=\frac{1}{V}<\hat{\phi}_{B}^{(n)}(\mathbf{q}) \hat{Q}_{x x}^{(B)}(-\mathbf{q})>_{0}= \\
& \frac{1}{\rho_{0} N} \int_{0}^{1} d x\left(\frac{1}{4}-\frac{3}{4} x^{2}\right) \frac{\sin ^{2}(q l(1-f) N x / 2)}{\sin ^{2}(q l x / 2)} \\
& \frac{1}{V}<\hat{Q}_{x x}^{(B)}(\mathbf{q}) \hat{Q}_{x x}^{(B)}(-\mathbf{q})>_{0}=\frac{1}{V}<\hat{Q}_{y y}^{(B)}(\mathbf{q}) \hat{Q}_{y y}^{(B)}(-\mathbf{q})>_{0}= \\
& \frac{1}{2 \rho_{0} N} \int_{0}^{1} d x\left(\frac{9}{16}\left(1-x^{2}\right)^{2}-\frac{3}{2}\left(1-x^{2}\right)+\frac{1}{2}\right) \frac{\sin ^{2}(q l(1-f) N x / 2)}{\sin ^{2}(q l x / 2)}{ }^{\prime}
\end{aligned}
$$




$$
\begin{aligned}
& \frac{1}{V}<\hat{Q}_{x x}^{(B)}(\mathbf{q}) \hat{Q}_{y y}^{(B)}(-\mathbf{q})>_{0}= \\
& \quad \frac{1}{2 \rho_{0} N} \int_{0}^{1} d x\left(\frac{27}{16}\left(1-x^{2}\right)^{2}-\frac{3}{2}\left(1-x^{2}\right)+\frac{1}{2}\right) \frac{\sin ^{2}(q l(1-f) N x / 2)}{\sin ^{2}(q l x / 2)}
\end{aligned}
$$

Finally in Eqs.(I.1-I.7) we take a limit of $q \rightarrow 0, N_{A}, N_{B} \rightarrow \infty$ such that $f=$ $N_{A} /\left(N_{A}+N_{B}\right)=$ const and $q l N=$ const. 


\section{References}

1) P.G. de Gennes Scaling Concept in Polymers Physics, (Cornell University, Ithaca, N.Y. 1979) p 103, 114-115.

2) P.G. de Gennes, J.Physique 31, 235 (1970);Scaling Concept in Polymer Physics , (Cornell University Ithaca, N.Y. 1979) p 259.

3) P.G. de Gennes, J.Physique Lett. 38, 441 (1977).

4) K.Binder, Phys.Rev A 29, 341 (1984).

5) J.F.Joanny J.Phys. A 11, L117 (1978).

6) P.Pfeuty and G.Toulouse, Introduction to the Renormalization Group and to Critical Phenomena (Wiley, New York, 1977) p 38.

7) D.J. Amit, Field Theory, The Renormalization Group, and Critical Phenomena, (World Scientific, Singapore 1984) p 87,109.

8) Ch. Herkt-Maetzky and J. Schelten, Phys.Rev.Lett 51, 896 (1983).

9) D.Schwahn, K. Mortensen and H. Yee-Madeira, Phys.Rev.Lett. 58, 1544 (1987).

10) F.S. Bates, J.H. Rosendale, P. Stepanek, T.P. Lodge, P.Wiltzius, G.H. Fredrickson and R.P. Hjelm Jr, Phys.Rev.Lett. 65, 1893 (1990).

11) G.Meier, B.Momper and E.W.Fischer,J.Chem.Phys. 97, 5884, (1992).

12) M.D. Gehlsen, J.H.Rosendale, F.S.Bates, G.D.Wignall, L. Hansen and K. Almdal, Phys.Rev.Lett. 68, 2452, (1992).

13) G. Meier, D.Schwahn, K. Mortensen and S. Janssen, Europhys. Lett. 22, 577 (1993).

14) R.Hołyst and T.A.Vilgis, J.Chem.Phys. 99, 4835 (1993).

15) R.Hołyst, Phys.Rev.Lett. 72, 2304 (1994).

16) M.G.Brereton and T.A.Vilgis, J.Phys. (France), 50, 245 (1989).

17) R.Hołyst and T.A.Vilgis, Phys.Rev. E 50, 2087 (1994). 
18) T.A.Vilgis and G.Meier, J.Phys. I (France), 4, 985 (1994).

19) A. Sariban and K.Binder, Macromolecules 21, 711 (1988).

20) J.L. Barrat and G. H. Fredrickson, J.Chem.Phys. 95, 1281 (1991).

21) H.Fried and K.Binder, J.Chem.Phys. 94, 8349 (1991); A.Weyersberg and T.A.Vilgis, Phys.Rev.E48, 377 (1993); A.Gauger, A.Weyersberg and T.Pakula, Macromol. Chem.Theory Simul. 2, 531 (1993).

22) L.Leibler, Macromolecules, 13, 1602 (1980).

23) G.H.Fredrickson an E.Helfand, J.Chem.Phys. 87, 697 (1987).

24) M.W.Matsen and M.Schick, Phys.Rev.Lett. 72, 2660 (1994).

25) M.W.Matsen and M.Schick, Macromolecules 27, 4014 (1994).

26) E.Helfand J.Chem.Phys. 62, 999 (1975).

27) K.M.Hong and J.Noolandi Macromolecules, 14, 727 (1981).

28) F.S.Bates and G.H.Fredrickson, Annu.Rev.Phys.Chem. 41, 525 (1990); F.S.Bates Science 251, 898 (1991).

29) G.E.Molau Block Copolymers (ed. S.L. Aggarawal) (Plenum Press, New York ) (1970)

30) E.L.Thomas et al, Macromolecules 19, 2197 (1986); D.S.Herman et al Macromolecules 20, 2940 (1987).

31) H.Hasegawa et al, Macromolecules 20, 1651 (1987).

32) E.L.Thomas et al , Nature 334, 598 (1988).

33) D.M. Anderson, H.T. Davis,J.C.C. Nitsche, L.E.Scriven, Adv.Chem.Phys. 77, 337 (1990).

34) D.Broseta and G.H.Fredrickson, J.Chem.Phys. 93, 2927 (1990).

35) R.Hołyst and M.Schick, J.Chem.Phys. 96, 7728 (1992).

36) M.W.Matsen and M.Schick, Macromolecules 26, 3878 (1993).

37) M.W.Matsen, Phys.Rev.Lett. 74, 4225 (1994). 
38) G.Gompper and M.Schick, Self Assembling Amphiphilic Systems, vol. 16 Phase Transitions and Critical Phenomena eds. C.Domb and J.L.Lebowitz, Academic Press (1994).

39) J. Stephenson J. Math. Phys. 11, 420 (1970); M. E. Fisher and B. Widom, J. Chem. Phys. 50, 3756 (1969).

40) G.H.Fredrickson and S.T.Milner, Phys.Rev.Lett. 67, 835 (1991).

41) G.Grinstein in Fundamental Problems in Statistical Mechanics IV ed E.G.D. Cohen (North Holland, Amsterdam) (1985).

42) S.F.Edwards and P.W.Anderson, J.Phys. F 5, 965 (1975).

43) M.Mezard, G.Parisi and M.A. Virasoro, Spin Glass Theory and Beyond, (World Scientific, Teaneck, NJ) (1987).

44) M.W.Matsen, J.Chem.Phys. 102, 3884 (1995).

45) M.D.Gehlsen, K.Almdal and F.S.Bates, Macromolecules 25, 939 (1992).

46a) M.E.Cates and J.M.Deutsch, J.Physique 47, 2121 (1986).

46b) M.G.Brereton and T.A.Vilgis, J.Phys.A 28, 1149 (1995).

47) A.Weyersberg and T.A.Vilgis, Phys.Rev.E 49, 3097 (1994).

48) J.F.Marko, Macromolecules 26, 1442 (1993).

49) M.Benmouna, R.Borsali and H.Benoit, J.Phys. II (France) 3, 1041 (1993).

50) R.Hołyst and M.Schick, J.Chem.Phys. 96, 730 (1992).

51) P.G. de Gennes and J.Prost, The Physics of Liquid Crystals, (Clarendon Press, Oxford) p57 (1993).

52) C.Singh, M.Goulian, A.J.Liu and G.H.Fredrickson, Macromolecules 27, 2974 (1994).

53) Recent Advances in Liquid Crystalline Polymers, edited by L. Chapoy, (Elsevier, New York 1985).

54) Polymer Liquid Crystals, edited by A. Ciferri, W.R. Kingbaum, and R.B. Meyer 
(Academic, New York 1982); Polymeric Liquid Crystals, edited by A. Blumstein, (Plenum Press, New York, 1985).

55) E. Bianchi, A. Ciferri, and A. Tealdi,Macromolecules 15, 1268 (1982);

C. Casagrande, M. Veyssië, and H. Finkelmann, J. Phys. Lett. 43, L671 (1982);

F. Auriemma, P. Corradini, A. Roviello, and M. Vacatello, Eur. Polym. J. 1, 57 (1989).

56) J.S. Moore and S.I. Stupp, Macromolecules 21, 1217 (1988).

57) P. Maïssa and P. Sixou, Liquid Crystals 5, 1861 (1989).

58) N. Saitô, K. Takahashi, and Y. Yunoki, J. Phys. Soc. Jap. 22, 219 (1967).

59) G.H. Fredrickson and L. Leibler, Macromolecules 23, 531 (1990).

60) R.Hołyst and M.Schick, J.Chem.Phys. 96, 721 (1992).

61) M.Lifschitz, J.Dudowicz and K.F.Freed, J.Chem.Phys 100, 3957 (1994).

62) M.Benmouna, T.A.Vilgis, M.Daoud and M.Benhamou, Macromolecules 27, 1172 (1994).

63) M.G.Brereton and T.A.Vilgis, Macromolecules 23, 2044 (1990).

64) R.Borsali and T.A.Vilgis, J.Chem.Phys. 93, 3610 (1990).

65) U.Genz and T.A.Vilgis, J.Chem.Phys. 101, 7101 (1994).

66) T.A.Vilgis and U.Genz, J. Phys I (France) 4, 1411 (1994).

67) G.H.Fredrickson and K.Binder, J.Chem.Phys. 91, 7265 (1989).

68) U.Genz and T.A.Vilgis, J.Chem.Phys. 101, 7111 (1994).

69) K. Freed, Renormalization Group Theory of Macromolecules, (Wiley, New York 1987), p21.

70) S.F. Edwards, Proc. Phys. Soc. (London) 88, 265 (1966).

71) K.S.Schweitzer and J.G.Curro, Phys.Rev.Lett. 60, 809 (1988).

72) J.-P. Hansen and I.R.MacDonald, Theory of Simple Liquids, 2nd edition (Academic Press) (1986). 
73) R.Evans, Adv. Phys. 28, 143 (1979).

74) F.S.Bates, P.Wiltzius and G.H.Fredrickson, Phys.Rev.Lett. 72, 2305 (1994).

75) M.Doi and S.F.Edwards, The Theory of Polymer Dynamics, (Clarendon Press, Oxford, 1986) p. 22,23.

76) T.Ohta and K.Kawasaki, Macromolecules 19, 2621 (1986).

77) S.Brazovskii, Sov.Phys.JETP 41, 85 (1975).

78) (a) K.Almdal, J.H.Rosendale, F.S.Bates, G.D.Wignall and G.H. Fredrickson, Phys.Rev.Lett. 65, 1112 (1990); (b) V.T.Bartels, M.Stamm, V.Abetz and K.Mortensen; Europhys.Lett. 31, 81 (1995).

79) D.A.Hajduk et al, Macromoecules, 27, 4063 (1994); M.Schulz et al, Phys.Rev.Lett. 73, 86 (1994).

80) H.Benoit and G.Hadziioannou, Macromolecules 21, 1449 (1988).

81) T.Pakula and S.Geyler, Macromolecules, 21, 1665 (1988).

82) S.Geyler and T.Pakula, Macromol. Chem. Rap. Commun., 9, 531 (1988).

83) K.Mori, H.Tanaka and T.Hashimoto, Macromolecules 20, 381 (1987).

84) A.M.Mayes and M.J. Olvera de la Cruz, J.Chem.Phys. 91, 7228 (1989).

85) A.M.Mayes and M.J. Olvera de la Cruz, J.Chem.Phys. 95, 4670 (1991).

86) M.W.Matsen and M.Schick, Macromolecules 27, 187 (1994).

87) G.Hadziioannou and A.Skoulios, Macromolecules 15, 258 (1982).

88) M.D.Gehlsen, K.Almdal and F.S.Bates, Macromolecules 25, 939 (1992).

89) R.W.Richards and J.L.Thomason, Macromolecules 16, 16 (1983).

90) E.I.Shakhnovich and A.M.Gutin, J.Phys. (Paris) 50, 1843 (1989).

91) Physics of Amphiphilic Layers, ed. J.Meunier, D.Langevin and N.Boccara (Springer, Berlin ) (1987).

92) L.Leibler, Macromolecules, 15, 1283 (1982).

93) G.Gompper and M.Schick, Phys.Rev.B 41, 9148 (1990). 
94) M.Mueller and M.Schick (unpublished).

95) M.P.Allen, G.T.Evans, D.Frenkel and B.M.Mulder, Adv. Chem.Phys. XXXVI, ed. I.Prigogine and S.A. Rice (John Wiley \& Sons Inc 1-166 (1993).

96) A.Poniewierski and R.Hołyst, Phys.Rev.Lett. 61, 2461 (1988); Phys.Rev. A 41, $6871(1990)$.

97) L.Onsager Proc. NY Acad. Sci. 51, 627 (1949).

98) F.Brochard, J.Jouffroy and P.Levinson, J.Phys. 45, 1125 (1984).

99) C.Shen and T.Kyu, J.Chem.Phys. 102, 556 (1995).

100) H.Chiu and T.Kyu, Macromolecules (in press) (1995).

101) M. Warner and P.J. Flory, J. Chem. Phys. 73, 6327 (1980). 


\section{Figure Captions}

Fig.1 The schematic picture of the unit cell of cubic bicontinuous double diamond phase formed in AB diblock copolymers. The junction of the A-B blocks are located at the surface shown in the figure. The surface defines the boundary between A rich and B rich domains. Symmetry Pnב̄m. The experimental studies suggest that the surface describing the boundary of domains in diblock copolymers is of constant mean curvature and is determined by the area minimization subject to fixed volume fraction of A and B blocks.

Fig.2 The schematic picture of the unit cell of gyroid structure formed in AB diblock copolymer systems. The junction points of the A-B blocks are located at the surface shown in the figure. Symmetry Ia $\overline{3} \mathrm{~d}$.

Fig.3 Phase diagram of the polystyrene-polyisoprene system ${ }^{30,31)}$. The bcc phase is the body center cubic phase, where droplets of the minority component in the diblock copolymer form an ordered lattice. The hex phase is the hexagonal phase where the parallel cylinders of the minority component surrounded by the majority component form a hexagonal lattice. The obdd phase is the ordered bicontinuous double diamond structure consisting of two channels each of diamond symmetry separated by the surface (Fig.1). ODT stands for orderdisorder transition. $\chi$ is the Flory interaction parameter, $N$ is the polimerization index and $f$ is the fraction of one component in the diblock copolymer system. The region of stability of the gyroid phase (Fig.2) which has been recently observed in this system is not shown here. After Ref. 28.

Fig.4 Bridge and loop configurations in the lamellar phase of multiblock copolymer system. After Ref. 44.

Fig.5 (a) On the left is shown the permitted configuration of an unknotted ring. It cannot turn into the knotted configuration shown on the right (not vice versa). 
(b) Two unknotted rings are shown in the allowed configuration. It cannot turn into the knotted configuration shown on the right. After Ref.46.

Fig.6 Microscopic model of an AB diblock copolymer molecule with different stiffness of the blocks. The size of the A segment, $b_{A}$, is much smaller than the size of the B segment $b_{B}$.After Ref.52.

Fig.7 The one-loop diagrams contributing to the two-body vertex function. The solid lines represent $1 / \Gamma_{2}^{(0)}$ while the higher order vertex functions are represented by points in the diagrams. The n-body vertex function has $\mathrm{n}$ lines emanating from the point representing this function. The dashed lines are not parts of the diagrams but are included only for clarity (according to Ref.(7)). Apart from $1 / \Gamma_{2}^{(0)}$, only $\Gamma_{3}^{(0)}$ and $\Gamma_{4}^{(0)}$ appear in the structure of the diagrams.

Fig. 8 The one-loop diagrams contributing to the three-body vertex function. The legend as in Fig. 1. Apart from $1 / \Gamma_{2}^{(0)}$ only $\Gamma_{3}^{(0)}$ and $\Gamma_{5}^{(0)}$ appear in the structure of the diagrams.

Fig.9 The plot of the logarithm of the scattering intensity versus the logarithm of the temperature difference. After Ref.11. Close to the critical point the intensity diverges according to the power law $\left|T-T_{c}\right|^{-\gamma}$. The experiment was performed here on the symmetric mixture of the PDMS and PEMS.

Fig.10 The inverse of the scattering intensity at zero $\mathrm{q}$ vector. The temperature $T_{x}$ marks the Ginzburg region where there crossover from the mean-field to the non-mean-field behavior. $T_{b}$ is the critical temperature on this plot. Please note that $T_{s, m f}$ (mean-field temperature) is not correctly identified (see text and Ref.15,74). After Ref.10. The experimental system studied in this paper is PI-PEP mixture.

Fig.11 The scattering intensity ${ }^{22}$ ) for the diblock copolymer in the random phase approximation for the system with composition $f=0.25$ as a function of 
$q^{2} R^{2}$, where $R^{2}=N l^{2} / 6$ is the radius of gyration; solid line $(\chi N=12.5)$, dashed line $(\chi N=16.0)$ and the dotted line $(\chi N=17.5)$. The peak in the scattering intensity is fixed and does not change with temperature, which is here proportional to $\sim 1 / \chi$

Fig.12 The phase diagram for the diblock copolymer system in the random phase approximation (after Ref.22 and Ref.23). The diagram is symmetric with respect to $\mathrm{f}=0.5$ (symmetric diblock copolymer). The transition to the body centered cubic (BCC) and hexagonal (HEX) structure is first order. The direct second order transition from the disordered phase (DIS) to the lamellar phase occurs only at $f=0.5$.

Fig.13 The phase diagram for the diblock copolymer system in the self consistent one-loop approximation (Hartree) (after Ref.23) for $N=10^{6}$. Compare with Fig.12; in the limit $N \rightarrow \infty$ the results of the Hartree approximation reduce to the random phase approximation. The dashed line marks the divergence of the scattering intensity in the random phase approximation (of course in the one-loop approximation for finite $N$ the structure factor does not diverge at this line).

Fig.14 Peak position, $q^{*}$, (in $\AA^{-1}$ ) versus the polimerization index, $N$ for the PEPPEE diblock copolymer system at fixed temperature T=296 K. After Ref.20. The vertical solid line indicates the microphase separation obtained in the theory. The solid line of slope $-1 / 2$ indicates the result of the random phase approximation. Open squares are the predictions of the one-loop approximation theory and full triangles are the experimental results ${ }^{78 a}$ ).

Fig.15 The phase diagram obtained in the self consistent field theory. Here $\mathrm{C}$ is the cubic bcc phase, $\mathrm{H}$ is the hexagonal phase, $\mathrm{L}$ is the lamellar phase and $\mathrm{G}$ is the gyroid phase (Fig.2). The diagram is symmetric with respect to reflection at 
$\mathrm{f}=0.5$. After Ref.24. The transition from the disordered to the lamellar phase is second order; exactly as in the random phase approximation. It is marked by a dot in the phase diagram. All the other transitions are first order.

Fig.16 The phase diagram of the diblock copolymer system with conformational asymmetry. The size of the A monomer, $l_{A}$, is equal to $\sqrt{10} l_{B}$, where $l_{B}$ is the size of the $\mathrm{B}$ monomer. The legend as in Fig.15, where $l_{A}=l_{B}$. Please note that conformational asymmetry does not change the topology of the phase diagram in this theory. After Ref[25].

Fig.17 The scattering intensity, calculated in the random phase approximation, for the symmetric ring diblocks and linear diblock copolymer system at infinite temperature $\chi=0$. The scattering from rings is less intense and the peak is at higher q vectors. After Ref.48.

Fig.18 The instability towards the ordered phase (divergence of the scattering intensity). The transition temperature $(\sim 1 / \chi)$ is approximately $40 \%$ lower in the rings than in the linear diblocks. After Ref.48.

Fig.19 Comparison of the radii of gyration for linear and ring (cyclic) symmetric diblock copolymers of length $N=20$. After Ref.47. Here $T_{c}$ is the temperature of the microphase separation (to the lamellar phase); $\epsilon$ is the enegy interaction parameter between the monomers and $a$ is the monomer size. The inverse of this temperature, in the random phase approximation, is shown (Fig.18) for $f=1 / 2$.

Fig.20 The distance between the centers of masses of A and B blocks in the ring diblock copolymer. After Ref.47. It indicates that the rings strongly stretch as the temperature is lowered towards the temperature of the microphase separation, $T_{c}$.

Fig.21 The radius of gyration for the single block in the ring copolymer $\alpha=A, B$. The 
blocks shrink as we lower the temperature, similarly as in the case of linear diblocks. $T_{c}$ is the temperature of the microphase separation. After Ref.47.

Fig.22 Phase diagram for the ABA triblock copolymer system calculated in the random phase approximation. Here BCC is the body center cubic phase, HCP is the hexagonal phase and LAM is the lamellar phase. After Ref.84. The two A blocks have the same number of A monomers.

Fig.23 Phase diagram of the tenary mixture at equal A and B homopolymer concentrations. Here only instability lines are marked. The three phase coexistance region is marked only schematically. In the region named lamellar there should be more different ordered phases, but they have not been studied, here. Also note that the point where all lines meet is the Lifshitz tricritical point. After Ref.35. See also Ref.34 and 36.

Fig.24 The same phase diagram as shown in Fig.23. Here the lines in the disordered phase shows the various stages of local ordering. LD denotes the disorder line, LF is the Lifshitz line for the $\Psi \Psi$ correlation function and LE and EG are the Lifshitz and the equimaxima lines of the $\eta \eta$ structure function. L is the Lifshitz tricritical point. The region between the LD and LF shows the region where the correlation function starts to oscillate showing a local ordering. After line LF the ordering becomes dominant and the peak in the structure functions appear. The region between the LG line and LE-EG is the region where the homopolymers order and where their structure functions develop a peak. At the EG line the homopolymer homopoymer structure function shows two peaks et equal height, one at zero q vector and one at non zero q vector. After Ref[35].

Fig.25 The stability limits of the disordered isotropic phase (I) against lamellar (L) (solid line) or nematic $(\mathrm{N})$ perturbations (dashed line) in the rigid-flexible diblock copolymer system for (a) $v_{B B} / \chi=0$, (b) $v_{B B} / \chi=0.4$, (c) $v_{B B} / \chi=3.0$. 
Dot denotes the tricritical point. After Ref.50.

Fig.26 The structure function $\tilde{G}_{\Psi \Psi}\left(q_{z}\right)$ (case $((\mathbf{a}),(\mathbf{c}))$ and its one dimensional Fourier transform (density-density correlation function) $G_{\Psi \Psi}(z)($ case $((\mathbf{b}),(\mathbf{d}))$. Here $\mathrm{N}$ is the number of monomers in a diblock copolymer, $\rho_{0}$ is the monomer density and $N_{B} l=(1-f) N l$ is the length of the rigid part. (a),(b)f=0.2, $\chi N=19.51$, and $v_{B B} / \chi=0$ (dashed line), and $\mathrm{f}=0.2, \chi N=19.51, v_{B B} / \chi=0.4$ (solid line). (c),(d) $\mathrm{f}=0.62, v_{B B} / \chi=0, \chi N=11.75$ (dashed line) and $\mathrm{f}=0.62, v_{B B} / \chi=3.0$, $\chi N=11.35$ (solid line). The parameters for the solid line are close to those of the tricritical point shown in Fig.25. After Ref.50.

Fig.27 The structure function $\tilde{G}_{Q Q}\left(q_{z}\right)$ versus $q_{z}$ and its one dimensional Fourier transform (nematic order parameter-nematic order parameter correlation function) $G_{Q Q}(z)$ versus z for $\mathrm{f}=0.62$. The parameters for the solid and dashed lines are as in Fig(26c,d). After Ref.50.

Fig.28 The structure function $\tilde{G}_{\Psi Q}\left(q_{z}\right)$ versus $q_{z}$ and its one dimensional Fourier transform (density-nematic order parameter correlation function) $G_{\Psi Q}(z)$ versus z for the same two systems as in Fig.26; (a),(b) $f=0.2 ;(\mathbf{c}),(\mathbf{d}) \mathrm{f}=0.62$. After Ref.50. 